A curvatura de Gauss-Kronecker de hipersuperfícies mínimas em espaços forma quadridimensionais

Felipe Rodolpho Sanches dos Santos

\author{
DisSERTAÇÃO APRESENTADA \\ $\mathrm{AO}$ \\ Instituto DE MATEMÁticA E EstatísticA \\ DA \\ UNIVERSIDADE DE SÃO PAUlO \\ PARA \\ OBTENÇÃO DO TÍTULO \\ $\mathrm{DE}$ \\ Mestre EM CiênCIAS
}

Programa: Matemática

Orientador: Prof ${ }^{\mathrm{a}}$. Dr ${ }^{\mathrm{a}}$. Rosa Maria dos Santos Barreiro Chaves 


\section{A curvatura de Gauss-Kronecker de hipersuperfícies mínimas em espaços forma quadridimensionais}

Esta versão da dissertação contém as correções e alterações sugeridas pela Comissão Julgadora durante a defesa da versão original do trabalho, realizada em 02/10/2020. Uma cópia da versão original está disponível no

Instituto de Matemática e Estatística da Universidade de São Paulo.

Comissão Julgadora:

- Prof $^{\mathrm{a}}$. Dr ${ }^{\mathrm{a}}$. Rosa Maria dos Santos Barreiro Chaves (orientadora) - IME-USP

- Prof. Dr. Luiz Amancio Machado de Sousa Junior - UNIRIO

- Prof. Dr. Márcio Fabiano da Silva - UFABC 


\section{Agradecimentos}

Primeiramente, agradeço à minha mãe, Sirlene, por todo amor e dedicação, além de seu apoio em todos os momentos e incentivo para que eu crescesse e buscasse meus sonhos. Agradeço também à minha avó, Neci, por estar sempre presente e tanto me ajudar.

Um agradecimento especial à minha orientadora, Rosa Maria dos Santos Barreiro Chaves, por ter me acolhido, um aluno de engenharia em seu primeiro ano de curso, para desenvolver um trabalho de iniciação científica em uma área tão interessante como a geometria diferencial. Agradeço por ter me aceitado, apesar de eu estar afastado da matemática e imerso no mundo da computação, como seu orientando neste projeto de mestrado, dedicando seu tempo e atenção para que desenvolvêssemos este trabalho.

Por fim, agradeço aos meus familiares, em especial minhas primas, Gabriela e Renata, por estarem presentes e ajudarem em meu crescimento. Agradeço também aos meus amigos pelo suporte e por me escutarem falar tanto de matemática, um tema que tanto adoro. 


\section{Resumo}

SANTOS, F. R. S. A curvatura de Gauss-Kronecker de hipersuperfícies mínimas em espaços forma quadridimensionais. 2020. 71 f. Dissertação (Mestrado) - Instituto de Matemática e Estatística, Universidade de São Paulo, São Paulo, 2020.

Nesse trabalho, estudamos os resultados obtidos por Asperti et al. [1] e Hasanis et al. [17] envolvendo a curvatura de Gauss-Kronecker de hipersuperfícies mínimas em espaços forma quadridimensionais. Apresentamos conceitos relativos ao estudo de variedades Riemannianas, assim como a técnica do referencial ortonormal móvel utilizada pelos dois artigos. Entre os resultados de [1], destaca-se para os casos Euclideano e hiperbólico uma versão local do resultado obtido por Cheng [4]. No caso esférico, obtemos uma isometria entre a imagem de uma imersão mínima de uma hipersuperfície completa com curvatura de Gauss-Kronecker constante não nula e o toro de Clifford. Apresentamos também dois teoremas referentes à classificação de hipersuperfícies mínimas completas em espaços forma quadridimensionais além de desenvolver os resultados presentes em [17].

Palavras-chave: curvatura de Gauss-Kronecker, hipersuperfícies mínimas, espaços forma quadridimensionais. 


\section{Abstract}

\section{SANTOS, F. R. S. The Gauss-Kronecker curvature of minimal hypersurfaces in}

four-dimensional space forms. 2020. 71 f. Dissertação (Mestrado) - Instituto de Matemática e Estatística, Universidade de São Paulo, São Paulo, 2020.

In this work, we study the results obtained by Asperti et al. [1] and Hasanis et al. [17] involving the Gauss-Kronecker curvature of minimal hypersurfaces in four-dimensional space forms. We present concepts related to the study of Riemannian manifolds, as well as the orthonormal frame field technique used by both articles. Among the results of [1], a local version of the result obtained by Cheng [4] stands out for the Euclidean and hyperbolic cases. In the spherical case, we obtain an isometry between the image of a minimal immersion of a complete hypersurface with non-zero constant Gauss-Kronecker curvature and the Clifford torus. We also present two theorems referring to the classification of complete minimal hypersurfaces in four-dimensional space forms, in addition to developing the results found in [17].

Keywords: Gauss-Kronecker curvature, minimal hypersurfaces, four-dimensional space forms. 


\section{Sumário}

$\begin{array}{ll}\text { Introdução } & 1\end{array}$

1 Preliminares $\quad 3$

1.1 Variedades Riemannianas . . . . . . . . . . . . . . . . . . . . 3

1.2 Equações de Estrutura . . . . . . . . . . . . . . . . . . . . . . . . . . . . . . . . .

1.3 Colchete de Lie e Derivada Covariante . . . . . . . . . . . . . . . . . . . 9

1.4 Distribuição . . . . . . . . . . . . . . . . . . . . . . . . . 14

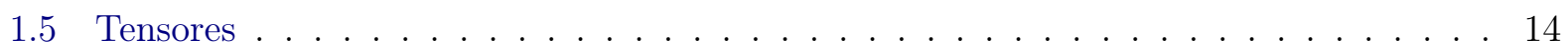

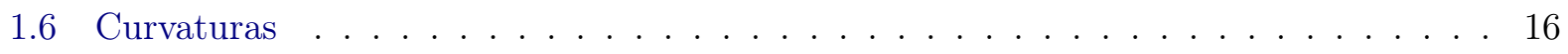

1.6.1 Operador de Curvatura . . . . . . . . . . . . . . . 16

1.6 .2 Curvatura Seccional . . . . . . . . . . . . . . . . . . . 19

1.6.3 Curvaturas de Ricci e Escalar . . . . . . . . . . . . . . . . . . . . . . 22

1.7 Operadores Diferenciais . . . . . . . . . . . . . . . . . . 22

1.8 Imersões Riemannianas . . . . . . . . . . . . . . . . . . . . . . . . . . 24

1.8.1 Segunda Forma Fundamental . . . . . . . . . . . . . . . 26

1.8.2 Curvaturas Principal, Média e de Gauss-Kronecker . . . . . . . . . . . . . . 27

1.8.3 Equações de Gauss, Codazzi e Ricci . . . . . . . . . . . . . . . . . . . . . . . . . . . . . . . . . . . . . . . . .

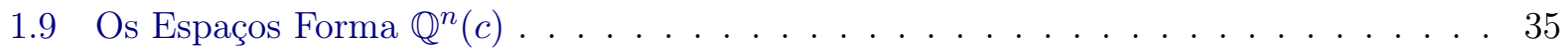

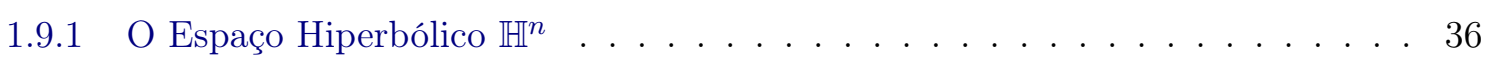

$1.9 .2 \quad$ O Espaço de De Sitter $\mathbb{S}_{1}^{n} \ldots \ldots \ldots \ldots \ldots$

2 Os Casos Euclideano $\mathbb{R}^{4}$ e Hiperbólico $\mathbb{H}^{4}$

3 O Caso Esférico $\mathbb{S}^{4} \quad 45$

4 Uma Classificação de Hipersuperfícies Mínimas de $\mathbb{Q}^{4}(c) \quad 57$

$\begin{array}{ll}\text { Referências Bibliográficas } & 69\end{array}$

$\begin{array}{ll}\text { Índice Remissivo } & 71\end{array}$ 


\section{Introdução}

Os espaços forma são variedades Riemannianas $n$-dimensionais completas, simplesmente conexas e com curvatura seccional constante $c$. Tais espaços, denotados por $\mathbb{Q}^{n}(c)$ neste texto, são mais simples e tem sua importância por serem clássicos e terem uma geometria mais simples. Temos que $\mathbb{Q}^{n}(c)$ é a esfera $\mathbb{S}^{n}(c)$ se $c>0, \mathbb{Q}^{n}(c)$ é o espaço Euclidiano $n$-dimensional $\mathbb{R}^{n}$ se $c=0$, e $\mathbb{Q}^{n}(c)$ é o espaço hiperbólico $n$-dimensional $\mathbb{H}^{n}(c)$, se $c<0$.

Diversos autores realizaram estudos envolvendo a curvatura de Gauss-Kronecker de hipersuperfícies em espaços forma, como por exemplo, Hadamard [16] mostrou, no caso Euclideano, que uma condição necessária e suficiente para uma hipersuperfície ser difeomorfa a uma esfera é que sua curvatura de Gauss-Kronecker seja diferente de zero em todos os pontos. Um resultado semelhante foi obtido por do Carmo e Warner [15] que estudaram hipersuperfícies compactas orientáveis na esfera.

Em [12], de Almeida e Brito classificaram as hipersuperfícies compactas mínimas $M^{3}$ na esfera $\mathbb{S}^{4}$ com curvatura de Gauss-Kronecker identicamente zero com uma hipótese adicional de que a segunda forma fundamental de $M^{3}$ nunca se anula, provando que tais hipersuperfícies são a fronteira de um tubo de uma imersão mínima com segunda forma fundamental que não se anula em nenhuma direção. Posteriormente, Ramanathan [25] conseguiu remover a hipótese sobre a segunda forma fundamental.

Mais recentemente em [4], Cheng provou que hipersuperfícies mínimas completas em $\mathbb{Q}^{4}(c)$, $c \leq 0$, com curvatura escalar limitada inferiormente e curvatura de Gauss-Kronecker constante, deve ter curvatura de Gauss-Kronecker identicamente zero.

Neste trabalho focamos na análise dos resultados obtidos por Asperti et al. [1] e Hasanis et al. [17], que motivados pelos resultados citados acima estudam a curvatura de Gauss-Kronecker de hipersuperfícies mínimas em espaços forma quadridimensionais. A seguir, faremos um breve resumo do que será abordado em cada capítulo.

O Capítulo 1 é dedicado à apresentação de algumas definições, técnicas e resultados mais gerais do estudo de variedades Riemannianas, que serão utilizados nos demais capítulos.

No Capítulo 2, nos focamos no estudo dos resultados para os casos Euclideano e hiperbólico, obtidos por Asperti et al. em [1]. O Lema 2.4 tem papel central na demonstração dos resultados apresentados, dando uma expressão para o Laplaciano da função $F=\ln |K|$, onde $K$ é a curvatura de Gauss-Kronecker de hipersuperfícies tridimensionais conexas minimamente imersas em $\mathbb{Q}^{4}(c)$. Com este lema e utilizando uma generalização do Princípio do Máximo de Yau [32], demonstramos os três resultados a seguir, onde o Teorema 2.6 é uma versão local para os resultados de Cheng [4] e Hasanis et al. [17]:

Teorema (Teorema 2.5) Seja $M^{3}$ uma variedade Riemanniana tridimensional conexa minimamente imersa em $\mathbb{Q}^{4}(c), c \leq 0$, com curvatura de Gauss-Kronecker $K$ nunca nula. Então a função $F=\ln |K|$ é uma das seguintes opções:

(i) super-harmônica se $c=0$; ou

(ii) fortemente super-harmônica se $c<0$.

Teorema (Teorema 2.6) Sejam $M^{3}$ uma variedade Riemanniana tridimensional conexa $e$ $f: M^{3} \rightarrow \mathbb{Q}^{4}(c), c \leq 0$, uma imersão minima com curvatura de Gauss-Kronecker $K$ constante. Então $K$ é identicamente zero. 
Teorema (Teorema 2.7) Seja $M^{3}$ uma variedade Riemanniana tridimensional completa minimamente imersa em $\mathbb{Q}^{4}(c), c \leq 0$, com curvatura de Ricci limitada inferiormente. Então a curvatura de Gauss-Kronecker K satisfaz inf $|K|=0$.

No Capítulo 3, utilizando o Lema 2.4 e uma generalização do Princípio do Máximo de Omori [23], estudamos o resultado apresentado a seguir, obtido por Asperti et al. em [1], que caracteriza as hipersuperfícies mínimas completas de $\mathbb{S}^{4}$ com curvatura de Gauss-Kronecker constante diferente de zero:

Teorema (Teorema 3.3) Seja $f: M^{3} \rightarrow \mathbb{S}^{4}$ uma imersão mínima de uma variedade Riemanniana completa com curvatura de Gauss-Kronecker constante $K \neq 0$. Então $f\left(M^{3}\right)$ é isométrico ao toro de Clifford $\mathbb{S}^{1}\left(\frac{\sqrt{3}}{3}\right) \times \mathbb{S}^{2}\left(\frac{\sqrt{6}}{3}\right)$.

No Capítulo 4, combinando os resultados presentes em Asperti et al. [1] e em Hasanis et al. $[17,18,19]$, enunciamos, assim como em [1], o Teorema 4.1 e o Teorema 4.2 que apresentam uma classificação de hipersuperfícies mínimas completas de $\mathbb{Q}^{4}(c)$. Recentemente, Dajczer et al. em $[9,10,11]$ trabalharam com subvariedades mínimas e completas com nulidade em espaços forma.

Optamos por trazer também no Capítulo 4 os resultados obtidos por Hasanis et al. em [17]. Os autores, motivados por Dajczer e Gromoll [8], estudam hipersuperfícies mínimas completas de $\mathbb{R}^{4}$ com curvatura de Gauss-Kronecker identicamente nula. Adicionando a hipótese de que a curvatura escalar é limitada inferiormente, concluem que tais hipersuperfícies são cilindros, obtendo o seguinte teorema:

Teorema (Teorema 4.7) Sejam $M^{3}$ uma variedade Riemanniana tridimensional completa orientada e $f: M^{3} \rightarrow \mathbb{R}^{4}$ uma imersão mínima isométrica com curvatura de Gauss-Kronecker identicamente zero e com segunda forma fundamental nunca nula. Se a curvatura escalar é limitada inferiormente, então $f\left(M^{3}\right)$ pode ser escrito como um produto Euclideano $L^{2} \times \mathbb{R}$, onde $L^{2}$ é uma superfície mínima completa de $\mathbb{R}^{3}$ com curvatura Gaussiana limitada inferiormente.

É importante ressaltar que existem em $\mathbb{R}^{3}$ superfícies mínimas completas com curvatura Gaussiana não limitada inferiormente [21], e desta forma, é possível construir cilindros sobre estas superfícies obtendo assim hipersuperfícies mínimas completas em $\mathbb{R}^{4}$, com curvatura de Gauss-Kronecker identicamente zero e curvatura escalar não limitada inferiormente. Existem diversos teoremas de decomposição como, por exemplo, os encontrados em Cheeger e Gromoll [3], Chodosh et al. [7], Innami [20] e Toponogov [30].

Utilizando o Teorema da Curvatura Principal de Smyth e Xavier [27], Hasanis et al. conseguem demonstrar o resultado obtido por Cheng [4], removendo a hipótese referente à curvatura escalar, enunciando o seguinte teorema:

Teorema (Teorema 4.9) Sejam $M^{3}$ uma variedade Riemanniana tridimensional completa orientada e $f: M^{3} \rightarrow \mathbb{R}^{4}$ uma imersão mínima isométrica com curvatura de Gauss-Kronecker constante. Então a curvatura de Gauss-Kronecker é identicamente zero. 


\section{Capítulo 1}

\section{Preliminares}

Neste capítulo apresentaremos definições e resultados mais genéricos sobre variedades Riemannianas que serão utilizados nos próximos capítulos, quando estivermos tratando especificamente de hipersuperfícies tridimensionais em espaço forma $\mathbb{Q}^{4}(c)$, com curvatura seccional constante $c$.

Tais resultados e definições foram extraídos de do Carmo [13, 14], sendo que alguns desenvolvimentos foram omitidos devido à grande vastidão do tema.

\subsection{Variedades Riemannianas}

O objetivo desta seção é apresentar a definição de variedade Riemanniana, utilizada no decorrer deste texto, além de alguns entes relacionados.

Definição 1.1.1 (Variedade Diferenciável) Uma variedade diferenciável de dimensão n é um conjunto $M$ e uma família de aplicações biunívocas $x_{\alpha}: U_{\alpha} \subset \mathbb{R}^{n} \rightarrow M$, de abertos $U_{\alpha}$ de $\mathbb{R}^{n}$ em $M$, tais que:

(i) $\bigcup_{\alpha} x_{\alpha}\left(U_{\alpha}\right)=M$.

(ii) Para todo par $\alpha, \beta$, com $x_{\alpha}\left(U_{\alpha}\right) \cap x_{\beta}\left(U_{\beta}\right)=W \neq \emptyset$, os conjuntos $x_{\alpha}^{-1}(W)$ e $x_{\beta}^{-1}(W)$ são abertos de $\mathbb{R}^{n}$ e as aplicações $x_{\beta}^{-1} \circ x_{\alpha}$ são diferenciáveis.

(iii) A família $\left\{\left(U_{\alpha}, x_{\alpha}\right)\right\}$ é maximal relativamente às condições (i) e (ii).

Ao longo deste texto, $M^{n}$ simbolizará uma variedade diferenciável $M$ de dimensão $n$.

Definição 1.1.2 (Aplicação Diferenciável) Sejam $M^{m}$ e $N^{n}$ variedades diferenciáveis. Uma aplicação $\varphi: M^{m} \rightarrow N^{n}$ é diferenciável em $p \in M^{m}$ se dada uma parametrização $\boldsymbol{y}: V \subset \mathbb{R}^{n} \rightarrow N^{n}$ em $\varphi(p)$ existe uma parametrização $\boldsymbol{x}: U \subset \mathbb{R}^{m} \rightarrow M^{m}$ em $p$, tal que $\varphi(\boldsymbol{x}(U)) \subset \boldsymbol{y}(V)$ e a aplicação

$$
\boldsymbol{y}^{-1} \circ \varphi \circ \boldsymbol{x}: U \subset \mathbb{R}^{m} \rightarrow \mathbb{R}^{n}
$$

é diferenciável em $\boldsymbol{x}^{-1}(p)$.

Definição 1.1.3 (Espaço Tangente) Seja $M^{n}$ uma variedade diferenciável. Uma aplicação diferenciável $\alpha:(-\epsilon, \epsilon) \rightarrow M^{n}$ é chamada uma curva (diferenciável) em $M^{n}$. Suponha que $\alpha(0)=p \in M^{n}$, e seja $\mathcal{D}$ o conjunto das funções de $M^{n}$, diferenciáveis em $p$. $O$ vetor tangente à curva $\alpha$ em $t=0$ é a função $\alpha^{\prime}(0): \mathcal{D} \rightarrow \mathbb{R}$ dada por

$$
\alpha^{\prime}(0) f=\left.\frac{d(f \circ \alpha)}{d t}\right|_{t=0}, \quad f \in \mathcal{D} .
$$

$U m$ vetor tangente em $p$ é o vetor tangente em $t=0$ de alguma curva $\alpha:(-\epsilon, \epsilon) \rightarrow M^{n}$ com $\alpha(0)=p$. O conjunto dos vetores tangentes a $M^{n}$ em $p$ será indicado por $T_{p} M$. O conjunto $T_{p} M$, 
com as operações usuais de funções, forma um espaço vetorial de dimensão n e é chamado de espaço tangente de $M^{n}$ em $p$.

Definição 1.1.4 (Imersão) Sejam $M^{m}$ e $N^{n}$ variedades diferenciáveis. Uma aplicação diferenciável $\varphi: M^{m} \rightarrow N^{n}$ é uma imersão se $d \varphi_{p}: T_{p} M \rightarrow T_{\varphi(p)} N$ é injetiva para todo $p \in M^{m}$. Observe-se que se $\varphi: M^{m} \rightarrow N^{n}$ é uma imersão, então $m \leq n$ e a diferença $(n-m)$ é chamada de codimensão da imersão $\varphi$.

Definição 1.1.5 (Fibrado Tangente) Seja $M^{n}$ uma variedade diferenciável e seja $T M=\left\{(p, v) ; p \in M, v \in T_{p} M\right\}$. É possível munir o conjunto TM com uma estrutura diferenciável (de dimensão 2n), e com tal estrutura TM será chamado de fibrado tangente de $M^{n}$. (Uma construção da estrutura diferenciável de TM pode ser encontrada no Exemplo 4.1 do Capítulo 0 de [13].)

Definição 1.1.6 (Campo de Vetores Tangente) Um campo de vetores tangentes $X$ em uma variedade diferenciável $M$ é uma correspondência que a cada ponto $p \in M$ associa um vetor $X(p) \in T_{p} M$. Em termos de aplicações, $X$ é uma aplicação de $M$ no fibrado tangente TM. $O$ campo é diferenciável se a aplicação $X: M \rightarrow T M$ é diferenciável. Neste texto indicaremos por $\mathcal{X}(M)$ o conjunto dos campos de vetores de classe $\mathcal{C}^{\infty}$ em $M$ e por $\mathcal{D}(M)$ o anel das funções reais de classe $\mathcal{C}^{\infty}$ definidas em $M$.

Considerando uma parametrização $\boldsymbol{x}: U \subset \mathbb{R}^{n} \rightarrow M^{n}$ e $p \in M^{n}$ é possivel escrever

$$
X(p)=\sum_{i=1}^{n} a_{i}(p) \frac{\partial}{\partial x_{i}}
$$

onde cada $a_{i}: U \rightarrow \mathbb{R}$ é uma função em $U$ e $\left\{\frac{\partial}{\partial x_{i}}\right\}$ é a base associada a $\boldsymbol{x}, i=1, \ldots, n$.

Definição 1.1.7 (Variedade Riemanniana) Uma métrica Riemanniana (ou estrutura Riemanniana) em uma variedade diferenciável $M$ é uma correspondência que associa a cada ponto $p$ de $M$ um produto interno $\langle,\rangle_{p}$ (isto é, uma forma bilinear simétrica, positiva definida) no espaço tangente $T_{p} M$, que varia diferenciavelmente com p no seguinte sentido: Se $X$ e $Y$ são campos diferenciáveis de vetores em $M$, então a função $p \mapsto\langle X, Y\rangle_{p}, p \in M$, é diferenciável em $M$. Uma variedade diferenciável $M$ munida de uma métrica Riemanniana é chamada de variedade Riemanniana.

Definição 1.1.8 (Variedade Riemanniana Completa) Uma variedade Riemanniana $M$ é dita completa se $M$ for completa como espaço métrico. (Esta é uma das equivalências demonstradas no Teorema de Hopf e Rinow em [13].)

A seguir apresentamos uma das técnicas utilizadas no estudo de variedades Riemannianas, o referencial ortonormal móvel.

Definição 1.1.9 (Referencial Ortonormal Móvel) Sejam $M^{n}$ uma variedade Riemanniana, $U \subset M^{n}$ um aberto de $M^{n}$. Sejam $e_{1}, \ldots, e_{n} n$ campos diferenciáveis de vetores em $U$, de tal modo que

$$
\left\langle e_{i}, e_{j}\right\rangle_{p}=\delta_{i j}, \quad \forall i, j=1, \ldots, n, \quad \forall p \in M^{n},
$$

onde $\delta_{i j}$ é o delta de Kronecker, ou seja,

$$
\delta_{i j}= \begin{cases}1, & \text { se } i=j \\ 0, & \text { se } i \neq j\end{cases}
$$

Um tal conjunto de campos de vetores é chamado de referencial ortonormal móvel em $U$.

Definição 1.1.10 (Correferencial Associado) Sejam $M^{n}$ uma variedade Riemanniana e $U \subset M^{n}$ um aberto de $M^{n}$. A partir do referencial ortonormal móvel $\left\{e_{1}, \ldots, e_{n}\right\}$ em $U$, é possivel definir em $U$ formas diferenciais lineares pela condição 


$$
\omega_{i}\left(e_{j}\right)=\delta_{i j}, \quad \forall i, j=1, \ldots, n,
$$

ou seja, em cada $p \in U$, a base $\left\{\left(\omega_{1}\right)_{p}, \ldots,\left(\omega_{n}\right)_{p}\right\}$ é a base dual da base $\left\{\left(e_{1}\right)_{p}, \ldots,\left(e_{n}\right)_{p}\right\}$. O conjunto das formas diferenciais $\left\{\omega_{1}, \ldots, \omega_{n}\right\}$ é chamado correferencial associado ao referencial ortonormal $\left\{e_{1}, \ldots, e_{n}\right\}$.

\subsection{Equações de Estrutura}

Será apresentada a seguir a construção das equações de estrutura para uma variedade Riemanniana. O desenvolvimento de tais equações é inspirado no caso de subvariedades no espaço Euclideano.

Inicia-se com dois lemas puramente algébricos que são necessários no decorrer do texto.

Lema 1.2.1 (Cartan) Seja $V$ um espaço vetorial de dimensão $n$. Sejam $\omega_{1}, \ldots, \omega_{r}: V \rightarrow \mathbb{R}$, $r \leq n$, formas lineares de $V$ linearmente independentes. Suponhamos que existam formas lineares $\theta_{1}, \ldots, \theta_{r}: V \rightarrow \mathbb{R}$ satisfazendo a seguinte condição: $\sum_{i=1}^{r} \omega_{i} \wedge \theta_{i}=0$. Então

$$
\theta_{i}=\sum_{j=1}^{r} a_{i j} \omega_{j}, \quad a_{i k}=a_{k i}, \quad \forall i, k=1, \ldots, r .
$$

Demonstração: Completemos as formas $\omega_{1}, \ldots, \omega_{r}$ em uma base $\omega_{1}, \ldots, \omega_{r}, \omega_{r+1}, \ldots, \omega_{n}$ de $V^{*}$ e escrevamos

$$
\theta_{i}=\sum_{j=1}^{r} a_{i j} \omega_{j}+\sum_{l=r+1}^{n} b_{i l} \omega_{l}, \quad \forall i=1, \ldots, r .
$$

Basta agora observar que a condição $\sum_{i=1}^{r} \omega_{i} \wedge \theta_{i}=0$ implica que

$$
\begin{aligned}
0 & =\sum_{i=1}^{r} \omega_{i} \wedge \theta_{i}=\sum_{i=1}^{r} \omega_{i} \wedge\left(\sum_{j=1}^{r} a_{i j} \omega_{j}+\sum_{l=r+1}^{n} b_{i l} \omega_{l}\right) \\
& =\sum_{i=1}^{r} \omega_{i} \wedge \sum_{j=1}^{r} a_{i j} \omega_{j}+\sum_{i=1}^{r} \omega_{i} \wedge \sum_{l=r+1}^{n} b_{i l} \omega_{l} \\
& =\sum_{\substack{i, j=1 \\
i<j}}^{r}\left(a_{i j}-a_{j i}\right) \omega_{i} \wedge \omega_{j}+\sum_{i=1}^{r} \sum_{l=r+1}^{n} b_{i l} \omega_{i} \wedge \omega_{l} .
\end{aligned}
$$

Como os $\omega_{k} \wedge \omega_{s}, k<s, \forall k, s=1, \ldots, n$, são linearmente independentes, conclui-se que $a_{i j}=a_{j i}$ e $b_{i l}=0, \forall i, j=1, \ldots, r, \forall l=r+1, \ldots, n$.

Lema 1.2.2 Sejam $V$ um espaço vetorial de dimensão $n, U \underset{a b}{\subset} V$ e $\omega_{1}, \ldots, \omega_{n}$ formas diferenciais linearmente independentes em $U$. Suponha que exista em $U$ um conjunto de 1-formas diferenciais $\omega_{i j}$ satisfazendo as condições:

$$
\omega_{i j}=-\omega_{j i}, \quad d \omega_{j}=\sum_{k=1}^{n} \omega_{k} \wedge \omega_{k j}, \quad \forall i, j=1, \ldots, n .
$$

Então um tal conjunto é único.

Demonstração: Suponhamos que exista um outro conjunto de formas $\bar{\omega}_{i j}$ com 


$$
\bar{\omega}_{i j}=-\bar{\omega}_{j i}, \quad d \omega_{j}=\sum_{k=1}^{n} \omega_{k} \wedge \bar{\omega}_{k j}, \quad \forall i, j=1, \ldots, n .
$$

Então $\sum_{k=1}^{n} \omega_{k} \wedge\left(\bar{\omega}_{k j}-\omega_{k j}\right)=0$, e pelo Lema 1.2.1,

$$
\bar{\omega}_{k j}-\omega_{k j}=\sum_{l=1}^{n} B_{k l}^{j} \omega_{l}, \quad B_{k i}^{j}=B_{i k}^{j}, \quad \forall i, j, k=1, \ldots, n .
$$

Observe que

$$
\bar{\omega}_{k j}-\omega_{k j}=\sum_{i=1}^{n} B_{k i}^{j} \omega_{i}=-\left(\bar{\omega}_{j k}-\omega_{j k}\right)=-\sum_{i=1}^{n} B_{j i}^{k} \omega_{i}
$$

e, como os $\omega_{i}$ são linearmente independentes, $B_{k i}^{j}=-B_{j i}^{k}$. Usando as simetrias obtidas, temos

$$
B_{j i}^{k}=-B_{k i}^{j}=-B_{i k}^{j}=B_{j k}^{i}=B_{k j}^{i}=-B_{i j}^{k}=-B_{j i}^{k}
$$

ou seja,

$$
B_{j i}^{k}=-B_{j i}^{k}
$$

Logo,

$$
B_{j i}^{k}=0 .
$$

Portanto, substituindo (1.2) em (1.1), concluímos que $\bar{\omega}_{k j}=\omega_{k j}, \forall k, j=1, \ldots, n$.

Lema 1.2.3 (Levi-Civitta) Escolhido um referencial ortonormal $\left\{e_{1}, \ldots, e_{n}\right\}$ em um aberto $U \subset M^{n}$ de uma variedade Riemanniana $M^{n}$, existe em $U$ um (único) conjunto de formas diferenciais $\omega_{i j}$ que são anti-simétricas, $\omega_{i j}=-\omega_{j i}$, e satisfazem

$$
d \omega_{i}=\sum_{j=1}^{n} \omega_{i j} \wedge \omega_{j}, \quad \forall i=1, \ldots, n
$$

Tais formas $\omega_{i j}$ são chamadas formas de conexão de $M^{n}$ no referencial ortonormal $\left\{e_{1}, \ldots, e_{n}\right\}$.

Demonstração: Façamos $d \omega_{i}\left(e_{j}, e_{k}\right)=A_{j k}^{i}$, isto é,

$$
d \omega_{i}=\sum_{\substack{j, k=1 \\ j<k}}^{n} A_{j k}^{i} \omega_{j} \wedge \omega_{k}=\sum_{\substack{j, k=1 \\ j<k}}^{n} A_{k j}^{i} \omega_{k} \wedge \omega_{j}, \quad A_{j k}^{i}=-A_{k j}^{i} .
$$

Queremos determinar funções $C_{i j}^{k}=-C_{j i}^{k}$ tais que as formas diferenciais

$$
\omega_{i j}=\sum_{k=1}^{n} C_{i j}^{k} \omega_{k}
$$

satisfaçam (1.3). Se tais funções existirem, teremos

$$
\omega_{i j}=\sum_{k=1}^{n} C_{i j}^{k} \omega_{k}=\sum_{k=1}^{n}-C_{j i}^{k} \omega_{k}=-\sum_{k=1}^{n} C_{j i}^{k} \omega_{k}=-\omega_{j i}
$$

e,

$$
d \omega_{i}=\sum_{\substack{j, k=1 \\ j<k}}^{n} A_{k j}^{i} \omega_{k} \wedge \omega_{j}=\sum_{j=1}^{n} \omega_{i j} \wedge \omega_{j}=\sum_{j=1}^{n}\left(\sum_{k=1}^{n} C_{i j}^{k} \omega_{k}\right) \wedge \omega_{j}
$$




$$
=\sum_{j, k=1}^{n} C_{i j}^{k} \omega_{k} \wedge \omega_{j}=\sum_{\substack{j, k=1 \\ j<k}}^{n}\left(C_{i j}^{k}-C_{i k}^{j}\right) \omega_{k} \wedge \omega_{j} .
$$

Como $\omega_{k} \wedge \omega_{j}, j<k$, são linearmente independentes, podemos, variando os índices, escrever as seguintes relações

$$
\left\{\begin{array}{l}
A_{k j}^{i}=C_{i j}^{k}-C_{i k}^{j} \\
A_{i k}^{j}=C_{j k}^{i}-C_{j i}^{k} \\
A_{i j}^{k}=C_{k j}^{i}-C_{k i}^{j} .
\end{array}\right.
$$

Somando membro a membro as igualdades acima, obteremos a seguinte condição necessária para a existência dos $C_{i j}^{k}$ :

$$
C_{i j}^{k}=\frac{1}{2}\left(A_{k j}^{i}+A_{i k}^{j}+A_{i j}^{k}\right)
$$

Definindo $C_{i j}^{k}$ como na expressão acima e as formas $\omega_{i j}$ por (1.4), basta verificar as condições pedidas. De fato,

$$
C_{i j}^{k}=\frac{1}{2}\left(A_{k j}^{i}+A_{i k}^{j}+A_{i j}^{k}\right)=\frac{1}{2}\left(-A_{j k}^{i}-A_{k i}^{j}-A_{j i}^{k}\right)=-\frac{1}{2}\left(A_{k i}^{j}+A_{j k}^{i}+A_{j i}^{k}\right)=-C_{j i}^{k},
$$

e,

$$
\begin{aligned}
\sum_{j=1}^{n} \omega_{i j} \wedge \omega_{j} & =\sum_{j=1}^{n}\left(\sum_{k=1}^{n} C_{i j}^{k} \omega_{k}\right) \wedge \omega_{j}=\sum_{j, k=1}^{n} C_{i j}^{k} \omega_{k} \wedge \omega_{j}=\sum_{\substack{j, k=1 \\
j<k}}^{n}\left(C_{i j}^{k}-C_{i k}^{j}\right) \omega_{k} \wedge \omega_{j} \\
& =\sum_{\substack{j, k=1 \\
j<k}}^{n}\left(\frac{1}{2}\left(A_{k j}^{i}+A_{i k}^{j}+A_{i j}^{k}\right)-\frac{1}{2}\left(A_{j k}^{i}+A_{i j}^{k}+A_{i k}^{j}\right)\right) \omega_{k} \wedge \omega_{j} \\
& =\sum_{\substack{j, k=1 \\
j<k}}^{n} A_{k j}^{i} \omega_{k} \wedge \omega_{j}=d \omega_{i} .
\end{aligned}
$$

A unicidade das formas $\omega_{i j}$ decorre do Lema 1.2.2.

Definição 1.2.4 (Formas de Curvatura) Sejam $M^{n}$ uma variedade diferenciável, definiremos

$$
\Omega_{i j}=d \omega_{i j}-\sum_{k=1}^{n} \omega_{i k} \wedge \omega_{k j} .
$$

As formas $\Omega_{i j}$ são chamadas formas de curvatura de $M^{n}$ no referencial ortonormal $\left\{e_{1}, \ldots, e_{n}\right\}$.

Teorema 1.2.5 (Equações de Estrutura) Sejam $M^{n}$ uma variedade Riemanniana e $\left\{e_{1}, \ldots, e_{n}\right\}$ um referencial ortonormal móvel em um aberto $U \subset M^{n}$. Sejam $\left\{\omega_{1}, \ldots, \omega_{n}\right\}$ o correferencial associado $e \omega_{i j}$ as formas de conexão de $U$ no referencial ortonormal $\left\{e_{1}, \ldots, e_{n}\right\}$. Então:

$$
\begin{gathered}
d \omega_{i}=\sum_{j=1}^{n} \omega_{i j} \wedge \omega_{j}, \\
d \omega_{i j}=\sum_{k=1}^{n} \omega_{i k} \wedge \omega_{k j}+\Omega_{i j} .
\end{gathered}
$$

Indicando as matrizes das formas $\omega_{i j}$ e $\Omega_{i j}$ por $W$ e $\Omega$, respectivamente, e o vetor coluna das formas $\omega_{i}$ por $\omega$, as equações acima podem ser escritas como a seguir: 


$$
\begin{gathered}
d \omega=W \wedge \omega, \\
d W=W \wedge W+\Omega .
\end{gathered}
$$

Demonstração: Os resultados são obtidos diretamente por (1.3) e (1.5).

Definição 1.2.6 (Mudança de Referencial) Seja $M^{n}$ uma variedade diferenciável e sejam $\left\{e_{1}, \ldots, e_{n}\right\}$ e $\left\{\bar{e}_{1}, \ldots, \bar{e}_{n}\right\}$ dois referenciais de $M^{n}$. Uma mudança de referencial $\left\{e_{1}, \ldots, e_{n}\right\}$ para $\left\{\bar{e}_{1}, \ldots, \bar{e}_{n}\right\}$ será dada por $e_{i}=\sum_{j=1}^{n} u_{i j} \bar{e}_{j}$, onde $\left(u_{i j}\right)=U$ é uma matriz de funções diferenciáveis em $M^{n}$. Além disso, $U$ é ortogonal, ou seja, $U U^{*}=\mathbb{1}_{n \times n}$, onde $U^{*}$ indica a matriz transposta de $U$.

Lema 1.2.7 Por uma mudança de referencial ortonormal $\left\{e_{1}, \ldots, e_{n}\right\}$ para $\left\{\bar{e}_{1}, \ldots, \bar{e}_{n}\right\}$ dada por $e_{i}=\sum_{j=1}^{n} u_{i j} \bar{e}_{j}$, a matriz das formas de conexão $W$ muda por

$$
W=d U U^{*}+U \bar{W} U^{*},
$$

e a matriz das formas de curvatura $\Omega$ muda por

$$
\Omega=U \bar{\Omega} U^{*},
$$

onde a barra indica a entidade correspondente no referencial ortonormal $\left\{\bar{e}_{1}, \ldots, \bar{e}_{n}\right\}$.

Demonstração: De $e_{i}=\sum_{j=1}^{n} u_{i j} \bar{e}_{j}$ decorre que $\omega_{i}=\sum_{j=1}^{n} u_{i j} \bar{\omega}_{j}$, isto é, $\omega=U \bar{\omega}$, e então $\bar{\omega}=U^{*} \omega$. Portanto,

$$
d \omega=d U \wedge \bar{\omega}+U d \bar{\omega}=d U \wedge\left(U^{*} \omega\right)+U(\bar{W} \wedge \bar{\omega})=\left(d U U^{*}+U \bar{W} U^{*}\right) \wedge \omega .
$$

Pelo lema da unicidade 1.2.2, decorre que

$$
W=d U U^{*}+U \bar{W} U^{*},
$$

o que demonstra (1.7). Para demonstrar (1.8), observemos que $d U U^{*}=-U(d U)^{*}$ e calcularemos $W \wedge W$ e $d W$. Daí:

$$
\begin{aligned}
W \wedge W & =\left(d U U^{*}+U \bar{W} U^{*}\right) \wedge\left(d U U^{*}+U \bar{W} U^{*}\right) \\
& =\left(d U U^{*}+U \bar{W} U^{*}\right) \wedge\left(-U(d U)^{*}+U \bar{W} U^{*}\right) \\
& =\left(d U U^{*}+U \bar{W} U^{*}\right) \wedge U\left(-(d U)^{*}+\bar{W} U^{*}\right) \\
& =(d U+U \bar{W}) \wedge\left(-(d U)^{*}+\bar{W} U^{*}\right) \\
& =-d U \wedge(d U)^{*}-U \bar{W} \wedge(d U)^{*}+d U \wedge \bar{W} U^{*}+U \bar{W} \wedge \bar{W} U^{*},
\end{aligned}
$$

e

$$
d W=-d U \wedge(d U)^{*}+d U \wedge \bar{W} U^{*}-U \bar{W} \wedge(d U)^{*}+U d \bar{W} U^{*}
$$

Portanto,

$$
\begin{aligned}
\Omega= & -W \wedge W+d W \\
= & d U \wedge(d U)^{*}+U \bar{W} \wedge(d U)^{*}-d U \wedge \bar{W} U^{*}-U \bar{W} \wedge \bar{W} U^{*} \\
& -d U \wedge(d U)^{*}+d U \wedge \bar{W} U^{*}-U \bar{W} \wedge(d U)^{*}+U d \bar{W} U^{*} \\
= & -U \bar{W} \wedge \bar{W} U^{*}+U d \bar{W} U^{*}=U(d \bar{W}-\bar{W} \wedge \bar{W}) U^{*}=U \bar{\Omega} U^{*},
\end{aligned}
$$

o que demonstra (1.8). 


\subsection{Colchete de Lie e Derivada Covariante}

Começamos apresentando um lema que nos permitirá definir um novo campo de vetores em uma variedade diferenciável a partir de dois outros. Tal lema nos garante que o novo campo está bem definido na variedade.

Lema 1.3.1 Sejam $X$ e $Y$ campos diferenciáveis em uma variedade diferenciável $M^{n}$. Então existe um único campo vetorial $Z$ tal que, para todo $f \in \mathcal{D}, Z f=(X Y-Y X) f$.

Demonstração: Primeiro provaremos que se $Z$ existe, ele é único. Admitamos, portanto, a existência de um tal $Z$. Sejam $p \in M^{n}$ e $\boldsymbol{x}: U \rightarrow M^{n}$ uma parametrização em $p$, e sejam

$$
X=\sum_{i=1}^{n} a_{i} \frac{\partial}{\partial x_{i}}, \quad Y=\sum_{i=1}^{n} b_{i} \frac{\partial}{\partial x_{i}}
$$

as expressões de $X$ e $Y$ nesta parametrização. Então para todo $f \in \mathcal{D}$,

$$
\begin{aligned}
& X Y f=X\left(\sum_{j=1}^{n} b_{j} \frac{\partial f}{\partial x_{j}}\right)=\sum_{i, j=1}^{n} a_{i} \frac{\partial b_{j}}{\partial x_{i}} \frac{\partial f}{\partial x_{j}}+\sum_{i, j=1}^{n} a_{i} b_{j} \frac{\partial^{2} f}{\partial x_{i} \partial x_{j}}, \\
& Y X f=Y\left(\sum_{j=1}^{n} a_{j} \frac{\partial f}{\partial x_{j}}\right)=\sum_{i, j=1}^{n} b_{i} \frac{\partial a_{j}}{\partial x_{i}} \frac{\partial f}{\partial x_{j}}+\sum_{i, j=1}^{n} a_{i} b_{j} \frac{\partial^{2} f}{\partial x_{i} \partial x_{j}} .
\end{aligned}
$$

Portanto, $Z$ é dado, na parametrização $x$, por

$$
Z f=X Y f-Y X f=\sum_{i, j=1}^{n}\left(a_{i} \frac{\partial b_{j}}{\partial x_{i}}-b_{i} \frac{\partial a_{j}}{\partial x_{i}}\right) \frac{\partial f}{\partial x_{j}},
$$

o que mostra a unicidade de $Z$.

Para demonstração da existência, defina-se $Z_{\alpha}$ em cada vizinhança coordenada $\boldsymbol{x}_{\alpha}\left(U_{\alpha}\right)$ de uma estrutura diferenciável $\left\{\left(U_{\alpha}, \boldsymbol{x}_{\alpha}\right)\right\}$ de $M^{n}$, pela expressão anterior. Por unicidade, $Z_{\alpha}=Z_{\beta}$ em $\boldsymbol{x}_{\alpha}\left(U_{\alpha}\right) \cap \boldsymbol{x}_{\beta}\left(U_{\beta}\right) \neq \emptyset$, o que permite definir $Z$ em toda a variedade $M^{n}$.

Com isso, temos a seguinte definição.

Definição 1.3.2 (Colchete de Lie) Sejam $X$ e $Y$ campos diferenciáveis em uma variedade diferenciável $M$. Então o campo vetorial dado por

$$
[X, Y]=X Y-Y X
$$

é chamado o colchete de Lie de $X$ e $Y$.

A proposição a seguir nos apresenta algumas das propriedade do colchete.

Proposição 1.3.3 Se $X, Y$ e $Z$ são campos diferenciáveis em $M, a, b$ são números reais, $f, g$ são funções diferenciáveis, e $\omega$ é uma 1-forma diferenciável então:

(a) $[X, Y]=-[Y, X]$ (anticomutatividade),

(b) $[a X+b Y, Z]=a[X, Z]+b[Y, Z]$ (linearidade),

(c) $[[X, Y], Z]+[[Y, Z], X]+[[Z, X], Y]=0$ (identidade de Jacobi),

(d) $[f X, g Y]=f g[X, Y]+f X(g) Y-g Y(f) X$,

(e) $d \omega(X, Y)=X(\omega(Y))-Y(\omega(X))-\omega([X, Y])$. 
Demonstração: Temos:

(a)

$$
\begin{aligned}
& {[X, Y]=X Y-Y X } \\
= & -(Y X-X Y)=-[Y, X] . \\
{[a X+b Y, Z]=} & (a X+b Y) Z-Z(a X+b Y) \\
= & a X Z+b Y Z-a Z X-b Z Y \\
= & a(X Z-Z X)+b(Y Z-Z Y)=a[X, Z]+b[Y, Z] .
\end{aligned}
$$

(c)

$$
\begin{aligned}
& {[[X, Y], Z]+[[Y, Z], X]+[[Z, X], Y] } \\
&=[X Y-Y X, Z]+[Y Z-Z Y, X]+[Z X-X Z, Y] \\
&= X Y Z-Z X Y-(Y X Z-Z Y X) \\
&+Y Z X-X Y Z-(Z Y X-X Z Y) \\
&+Z X Y-Y Z X-(X Z Y-Y X Z)=0 .
\end{aligned}
$$

$$
\begin{aligned}
{[f X, g Y] } & =f X(g Y)-g Y(f X) \\
& =f(X(g) Y+g X Y)-g(Y(f) X+f Y X) \\
& =f g(X Y-Y X)+f X(g) Y-g Y(f) X \\
& =f g[X, Y]+f X(g) Y-g Y(f) X .
\end{aligned}
$$

Para o item (e), como $\omega$ é uma 1-forma, ela pode ser expressa localmente como soma de termos da forma $g d f \operatorname{com} f$ e $g$ funções diferenciáveis. Desta forma, basta provar para o caso $\omega=g d f$ que o resultado segue por linearidade. Pelo lado esquerdo de (e) temos

$$
\begin{aligned}
d \omega(X, Y) & =d(g d f)(X, Y)=d g \wedge d f(X, Y) \\
& =d g(X) d f(Y)-d g(Y) d f(X) \\
& =X g Y f-Y g X f
\end{aligned}
$$

Já pelo lado direito, temos

$$
\begin{aligned}
X(\omega(Y))-Y(\omega(X)) & -\omega([X, Y]) \\
& =X(g d f(Y))-Y(g d f(X))-g d f([X, Y]) \\
& =X(g Y f)-Y(g X f)-g[X, Y] f \\
& =X g Y f+g X Y f-Y g X f-g Y X f-g X Y f+g Y X f \\
& =X g Y f-Y g X f
\end{aligned}
$$

e concluímos a igualdade afirmada em (e).

Até o momento, podemos derivar funções mas não campos de vetores. Graças às formas de conexão $\omega_{i j}$ apresentadas no Lema 1.2 .3 e com a proposição a seguir, uma variedade Riemanniana estará munida de uma derivação de campos de vetores.

Proposição 1.3.4 (Derivada Covariante) Sejam X e Y campos diferenciáveis de vetores em $M^{n}$ e seja $\left\{e_{1}, \ldots, e_{n}\right\}$ um referencial ortonormal em um aberto $U \subset M^{n}$. Suponhamos que $Y=\sum_{i=1}^{n} y_{i} e_{i}$ e façamos

$$
\nabla_{X} Y=\sum_{i=1}^{n}\left(d y_{i}(X)+\sum_{j=1}^{n} \omega_{j i}(X) y_{j}\right) e_{i} .
$$

Então $\nabla_{X} Y$, chamada derivada covariante de $Y$ em relação a $X$, é independente do referencial ortonormal $\left\{e_{1}, \ldots, e_{n}\right\}$ e, portanto, globalmente definida em $M^{n}$. 
Demonstração: Será conveniente utilizar a notação matricial estabelecida anteriormente na Definição 1.2.6 e no Lema 1.2.7, com as seguintes adições: $e=\left(e_{1}, \ldots, e_{n}\right)$ será uma matriz linha e $y=\left(y_{1}, \ldots, y_{n}\right)$ será uma matriz coluna; assim $Y=e y$. Se $\bar{e}$ é um outro referencial ortonormal, façamos $e=\bar{e} U^{*}$; assim $y=U \bar{y}$. Com uma tal notação, a equação (1.9) se escreve

$$
\nabla_{X} Y=e\left(d y(X)+W^{*}(X) y\right) .
$$

Como $X$ não irá interferir nos cálculos, vamos omiti-lo nas expressões abaixo. Inicialmente observemos que

$$
d y=d U \bar{y}+U d \bar{y},
$$

e que, da equação (1.7), vem

$$
W^{*} y=U(d U)^{*} y+U \bar{W}^{*} U^{*} y=-d U U^{*} y+U \bar{W}^{*} U^{*} y=-d U \bar{y}+U \bar{W}^{*} \bar{y} .
$$

Portanto,

$$
\begin{aligned}
e\left(d y+W^{*} y\right) & =e\left(d U \bar{y}+U d \bar{y}-d U \bar{y}+U \bar{W}^{*} \bar{y}\right) \\
& =e U d \bar{y}+e U \bar{W}^{*} \bar{y}=\bar{e}\left(d \bar{y}+\bar{W}^{*} \bar{y}\right),
\end{aligned}
$$

o que mostra que (1.9) não depende da escolha do referencial ortonormal $\left\{e_{1}, \ldots, e_{n}\right\}$.

Corolário 1.3.5 A derivada covariante permite interpretar geometricamente as formas de conexão. Com efeito, de (1.9) decorre que, para todo campo $X$,

$$
\left\langle\nabla_{X} e_{i}, e_{j}\right\rangle=\omega_{i j}(X)
$$

Portanto,

$$
\omega_{i j}\left(e_{k}\right)=\left\langle\nabla_{e_{k}} e_{i}, e_{j}\right\rangle
$$

$e$

$$
\nabla_{e_{i}} e_{j}=\sum_{k=1}^{n}\left\langle\nabla_{e_{i}} e_{j}, e_{k}\right\rangle e_{k}=\sum_{k=1}^{n} \omega_{j k}\left(e_{i}\right) e_{k}
$$

Os primeiros quatro itens da proposição a seguir garantem que a derivada covariante satisfaz algumas propriedades esperadas.

Proposição 1.3.6 (Propriedades da Derivada Covariante) Sejam $M^{n}$ uma variedade diferenciável, $X, Y, Z$ campos diferenciáveis de vetores em $M^{n}, f, g$ funções diferenciáveis em $M^{n}$ e a, $b$ números reais. Então:

(a) $\nabla_{f X+g Z} Y=f \nabla_{X} Y+g \nabla_{Z} Y$,

(b) $\nabla_{X}(a Y+b Z)=a \nabla_{X} Y+b \nabla_{X} Z$,

(c) $\nabla_{X}(f Y)=f \nabla_{X} Y+X(f) Y$,

(d) $\left\langle\nabla_{X} Y, Z\right\rangle+\left\langle Y, \nabla_{X} Z\right\rangle=X(\langle Y, Z\rangle)$,

(e) $\nabla_{X} Y-\nabla_{Y} X=[X, Y]$,

(f) Se $p \in M^{n},\left(\nabla_{X} Y\right)(p)$ só depende do valor de $X$ no ponto $p$ e dos valores de $Y$ ao longo de uma curva parametrizada $\alpha:(-\epsilon, \epsilon) \rightarrow M^{n}$, com $\alpha(0)=p$ e $\alpha^{\prime}(0)=X(p)$.

Demonstração: Seja $\left\{e_{1}, \ldots, e_{n}\right\}$ um referencial ortonormal de $M^{n}$ e escrevamos $X=\sum_{i=1}^{n} x_{i} e_{i}$, $Y=\sum_{i=1}^{n} y_{i} e_{i}, Z=\sum_{i=1}^{n} z_{i} e_{i}$. Com isso, e utilizando (1.9), temos: 
(a)

$$
\begin{aligned}
\nabla_{f X+g Z} Y & =\sum_{i=1}^{n}\left(d y_{i}(f X+g Z)+\sum_{j=1}^{n} \omega_{j i}(f X+g Z) y_{j}\right) e_{i} \\
& =\sum_{i=1}^{n}\left(f d y_{i}(X)+g d y_{i}(Z)+f \sum_{j=1}^{n} \omega_{j i}(X) y_{j}+g \sum_{j=1}^{n} \omega_{j i}(Z) y_{j}\right) e_{i} \\
& =f \sum_{i=1}^{n}\left(d y_{i}(X)+\sum_{j=1}^{n} \omega_{j i}(X) y_{j}\right) e_{i}+g \sum_{i=1}^{n}\left(d y_{i}(Z)+\sum_{j=1}^{n} \omega_{j i}(Z) y_{j}\right) e_{i} \\
& =f \nabla_{X} Y+g \nabla_{Z} Y .
\end{aligned}
$$

(b)

$$
\begin{aligned}
\nabla_{X}(a Y+b Z) & =\sum_{i=1}^{n}\left(d\left(a y_{i}+b z_{i}\right)(X)+\sum_{j=1}^{n} \omega_{j i}(X)\left(a y_{j}+b z_{j}\right)\right) e_{i} \\
& =\sum_{i=1}^{n}\left(a d y_{i}(X)+b d z_{i}(X)+a \sum_{j=1}^{n} \omega_{j i}(X) y_{j}+b \sum_{j=1}^{n} \omega_{j i}(X) z_{j}\right) e_{i} \\
& =a \sum_{i=1}^{n}\left(d y_{i}(X)+\sum_{j=1}^{n} \omega_{j i}(X) y_{j}\right) e_{i}+b \sum_{i=1}^{n}\left(d z_{i}(X)+\sum_{j=1}^{n} \omega_{j i}(X) z_{j}\right) e_{i} \\
& =a \nabla_{X} Y+b \nabla_{X} Z .
\end{aligned}
$$

(c)

$$
\begin{aligned}
\nabla_{X}(f Y) & =\sum_{i=1}^{n}\left(d\left(f y_{i}\right)(X)+\sum_{j=1}^{n} \omega_{j i}(X)\left(f y_{j}\right)\right) e_{i} \\
& =\sum_{i=1}^{n}\left(y_{i} d f(X)+f d y_{i}(X)+f \sum_{j=1}^{n} \omega_{j i}(X) y_{j}\right) e_{i} \\
& =f \sum_{i=1}^{n}\left(d y_{i}(X)+\sum_{j=1}^{n} \omega_{j i}(X) y_{j}\right) e_{i}+X(f) \sum_{i=1}^{n} y_{i} e_{i} \\
& =f \nabla_{X} Y+X(f) Y .
\end{aligned}
$$

(d) $\left\langle\nabla_{X} Y, Z\right\rangle+\left\langle Y, \nabla_{X} Z\right\rangle=\left\langle\sum_{i=1}^{n}\left(d y_{i}(X)+\sum_{j=1}^{n} \omega_{j i}(X) y_{j}\right) e_{i}, \sum_{i=1}^{n} z_{i} e i\right\rangle$

$$
\begin{aligned}
& +\left\langle\sum_{i=1}^{n} y_{i} e i, \sum_{i=1}^{n}\left(d z_{i}(X)+\sum_{j=1}^{n} \omega_{j i}(X) z_{j}\right) e_{i}\right\rangle \\
= & \sum_{i=1}^{n}\left(d y_{i}(X)+\sum_{j=1}^{n} \omega_{j i}(X) y_{j}\right) z_{i}+\sum_{i=1}^{n}\left(d z_{i}(X)+\sum_{j=1}^{n} \omega_{j i}(X) z_{j}\right) y_{i} \\
= & \sum_{i=1}^{n}\left(z_{i} d y_{i}(X)+y_{i} d z_{i}(X)\right)+\omega_{11}(X) y_{1} z_{1}+\omega_{21}(X) y_{2} z_{1}+\ldots \\
& +\omega_{n 1}(X) y_{n} z_{1}+\omega_{12}(X) y_{1} z_{2}+\cdots+\omega_{n n}(X) y_{n} z_{n}+\omega_{11}(X) z_{1} y_{1} \\
& \left.+\omega_{21}(X) z_{2} y_{1}+\cdots+\omega_{n 1}(X) z_{n} y_{1}+\omega_{12}(X) z_{1} y_{2}+\cdots+\omega_{n n}(X) z_{n} y_{n}\right) \\
= & \sum_{i=1}^{n} d\left(y_{i} z_{i}\right)(X)=X\left(\sum_{i=1}^{n} y_{i} z_{i}\right)=X\left(\left\langle\sum_{i=1}^{n} y_{i} e_{i}, \sum_{i=1}^{n} z_{i} e_{i}\right\rangle\right) \\
= & X(\langle Y, Z\rangle) .
\end{aligned}
$$


Para o item (e), começamos primeiro provando o resultado para dois elementos quaisquer do referencial ortonormal. Sabemos que

$$
\nabla_{e_{i}} e_{j}-\nabla_{e_{j}} e_{i}=\sum_{k=1}^{n} \omega_{k}\left(\nabla_{e_{i}} e_{j}-\nabla_{e_{j}} e_{i}\right) e_{k}
$$

$\mathrm{e}$

$$
\left[e_{i}, e_{j}\right]=\sum_{k=1}^{n} \omega_{k}\left(\left[e_{i}, e_{j}\right]\right) e_{k} .
$$

Portanto, basta mostrarmos que $\omega_{k}\left(\nabla_{e_{i}} e_{j}-\nabla_{e_{j}} e_{i}\right)=\omega_{k}\left(\left[e_{i}, e_{j}\right]\right), \forall k=1, \ldots, n$, para concluirmos que $\nabla_{e_{i}} e_{j}-\nabla_{e_{j}} e_{i}=\left[e_{i}, e_{j}\right]$. De fato, por (1.6), temos

$$
\begin{aligned}
d \omega_{k}\left(e_{i}, e_{j}\right) & =\sum_{l=1}^{n} \omega_{k l} \wedge \omega_{l}\left(e_{i}, e_{j}\right) \\
& =\sum_{l=1}^{n}\left(\omega_{k l}\left(e_{i}\right) \omega_{l}\left(e_{j}\right)-\omega_{k l}\left(e_{j}\right) \omega_{l}\left(e_{i}\right)\right) \\
& =\omega_{k j}\left(e_{i}\right)-\omega_{k i}\left(e_{j}\right) .
\end{aligned}
$$

Pela propriedade (e) da Proposição 1.3.3, temos

$$
d \omega_{k}\left(e_{i}, e_{j}\right)=e_{i}\left(\omega_{k}\left(e_{j}\right)\right)-e_{j}\left(\omega_{k}\left(e_{i}\right)\right)-\omega_{k}\left(\left[e_{i}, e_{j}\right]\right),
$$

ou seja,

$$
\omega_{k}\left(\left[e_{i}, e_{j}\right]\right)=-d \omega_{k}\left(e_{i}, e_{j}\right)
$$

Por (1.11), (1.12) e (1.13), temos

$$
\begin{aligned}
\omega_{k}\left(\nabla_{e_{i}} e_{j}-\nabla_{e_{j}} e_{i}\right) & =\omega_{k}\left(\nabla_{e_{i}} e_{j}\right)-\omega_{k}\left(\nabla_{e_{j}} e_{i}\right) \\
& =\omega_{k}\left(\sum_{l=1}^{n} \omega_{j l}\left(e_{i}\right) e_{l}\right)-\omega_{k}\left(\sum_{l=1}^{n} \omega_{i l}\left(e_{j}\right) e_{l}\right) \\
& =\sum_{l=1}^{n} \omega_{j l}(e i) \omega_{k}\left(e_{l}\right)-\sum_{l=1}^{n} \omega_{i l}(e j) \omega_{k}\left(e_{l}\right) \\
& =\omega_{j k}\left(e_{i}\right)-\omega_{i k}\left(e_{j}\right)=-d \omega_{k}\left(e_{i}, e_{j}\right) \\
& =\omega_{k}\left(\left[e_{i}, e_{j}\right]\right) .
\end{aligned}
$$

Finalmente, temos

$$
\begin{aligned}
\nabla_{X} Y-\nabla_{Y} X & =\sum_{i, j=1}^{n}\left(\nabla_{x_{i} e_{i}} y_{j} e_{j}-\nabla_{y_{j} e_{j}} x_{i} e_{i}\right) \\
& =\sum_{i, j=1}^{n}\left(x_{i} y_{j} \nabla_{e_{i}} e_{j}+x_{i} e_{i}\left(y_{j}\right) e_{j}-x_{i} y_{j} \nabla_{e_{j}} e_{i}-y_{j} e_{j}\left(x_{i}\right) e_{i}\right) \\
& =\sum_{i, j=1}^{n}\left(x_{i} y_{j}\left[e_{i}, e_{j}\right]+x_{i} e_{i}\left(y_{j}\right) e_{j}-y_{j} e_{j}\left(x_{i}\right) e_{i}\right) \\
& =\sum_{i, j=1}^{n}\left[x_{i} e_{i}, y_{j} e_{j}\right]=\left[\sum_{i=1}^{n} x_{i} e_{i}, \sum_{j=1}^{n} y_{j} e_{j}\right] \\
& =[X, Y] .
\end{aligned}
$$


Para o item (f), basta observar a expressão de $\nabla_{X} Y$ em (1.9).

\subsection{Distribuição}

Apresentamos agora algumas definições e resultados da teoria de variedades que serão utilizados na demonstração do Teorema 4.7.

Definição 1.4.1 (Distribuição) Seja $M^{m}$ uma variedade Riemanniana de dimensão $m=n+k e$ suponha que para cada $p \in M^{m}$ está associado um subespaço n-dimensional $D_{p}$ de $T_{p} M$. Além disso, suponha que em uma vizinhança $U$ de cada $p \in M^{m}$ existam $n$ campos de vetores $\mathcal{C}^{\infty}$ linearmente independentes $X_{1}, \ldots, X_{n}$, que formam uma base de $D_{q}$, para todo $q \in U$. Então dizemos que $D$ é uma distribuição $\mathcal{C}^{\infty}$ de dimensão $n$ em $M^{m}$ e que $X_{1}, \ldots, X_{n}$ é uma base local de $D$.

Definição 1.4.2 (Distribuição Involutiva) Sejam $M^{m}$ uma variedade Riemanniana de dimensão $m=n+k$ e $D$ uma distribuiçãa $\mathcal{C}^{\infty}$ de dimensão $n$ em $M^{m}$. Dizemos que a distribuição $D$ é involutiva, se existe uma base local $X_{1}, \ldots, X_{n}$ em uma vizinhança de cada ponto $p \in M^{m}$ tal que

$$
\left[X_{i}, X_{j}\right]=\sum_{k=1}^{n} c_{i j}^{k} X_{k}, \quad 1 \leq i, j \leq n,
$$

onde $c_{i j}^{k}$ são funções $\mathcal{C}^{\infty}$ na vizinhança.

Definição 1.4.3 (Variedade Integral) Sejam $M^{m}$ uma variedade Riemanniana de dimensão $m=n+k$ e $D$ uma distribuição $\mathcal{C}^{\infty}$ de dimensão $n$ em $M^{m}$. Suponha que $N$ é uma subvariedade conexa de $M$ tal que para cada $p \in N$ temos que $T_{p} N \subset D_{p}$. Nesta situação, dizemos que $N$ é uma variedade integral de $D$.

Definição 1.4.4 (Distribuição Completamente Integrável) Sejam $M^{m}$ uma variedade Riemanniana de dimensão $m=n+k$ e $D$ uma distribuição $\mathcal{C}^{\infty}$ de dimensão $n$ em $M^{m}$. Se para cada $p \in M^{m}$ existe uma variedade integral $N$ de $D$ passando por $p$ tal que a dimensão de $N$ seja $n$, dizemos que $D$ é completamente integrável.

Enunciamos agora o Teorema de Frobenius. Omitimos sua demonstração mas ela pode ser encontrada em Boothby [2].

Teorema 1.4.5 (Frobenius) Uma distribuição D em uma variedade $M$ é completamente integrável se, e somente se, ela é involutiva.

\subsection{Tensores}

Esta seção faz uma apresentação do conceito de tensores em uma variedade Riemanniana, além de definir dois conceitos de diferenciação.

Definição 1.5.1 (Tensor) Seja $M^{n}$ uma variedade Riemanniana. Um tensor de ordem $\mathrm{r}$ em $M^{n}$ é uma correspondência $F$ que a cada ponto $p \in M^{n}$ associa uma forma $r$-linear

$$
F_{p}: \underbrace{T_{p} M \times \ldots T_{p} M}_{r \text { fatores }} \rightarrow \mathbb{R} .
$$

Um tensor $F$ é diferenciável em $p \in M^{n}$ se escolhido um referencial ortonormal $\left\{e_{1}, \ldots, e_{n}\right\}$, em uma vizinhança $U$ de $p$, as funções $F_{i_{1} i_{2} \ldots i_{r}}$, chamadas de componentes do tensor $F$ no referencial ortonormal $\left\{e_{1}, \ldots, e_{n}\right\}$, e dadas por

$$
F_{i_{1} i_{2} \ldots i_{r}}(q)=F_{q}\left(e_{i_{1}}, e_{i_{2}}, \ldots, e_{i_{r}}\right), \quad i_{1}, i_{2}, \ldots, i_{r}=1, \ldots, n, \quad q \in U
$$


são diferenciáveis em . Note que esta condição não depende da escolha do referencial ortonormal $\left\{e_{1}, \ldots, e_{n}\right\} . F$ é diferenciável em $M^{n}$ se é diferenciável para todo $p \in M^{n}$. Neste texto, só consideraremos tensores diferenciáveis e por isso omitiremos o adjetivo.

A seguir, será definido uma forma de estender o conceito de diferencial para tensores em uma variedade Riemanniana.

Definição 1.5.2 (Diferencial Covariante) Sejam $F$ um tensor de ordem $r$ em uma variedade Riemanniana $M^{n}, p \in M^{n}$ e $\left\{e_{1}, \ldots, e_{n}\right\}$ um referencial ortonormal em uma vizinhança $U$ de $p$. $A$ diferencial covariante $\nabla F$ é um tensor de ordem $r+1$ definido da seguinte maneira. As componentes

$$
F_{i_{1} i_{2} \ldots i_{r} ; j}=\nabla F\left(e_{i_{1}}, e_{i_{2}}, \ldots, e_{i_{r}}, e_{j}\right), \quad i_{1}, i_{2}, \ldots, i_{r}, j=1, \ldots, n,
$$

de $\nabla F$ no referencial ortonormal $\left\{e_{1}, \ldots, e_{n}\right\}$ são dadas por

$$
\sum_{j=1}^{n} F_{i_{1} i_{2} \ldots i_{r} ; j} \omega_{j}=d F_{i_{1} i_{2} \ldots i_{r}}+\sum_{j=1}^{n} F_{j i_{2} \ldots i_{r}} \omega_{j i_{1}}+\sum_{j=1}^{n} F_{i_{1} j \ldots i_{r}} \omega_{j i_{2}}+\cdots+\sum_{j=1}^{n} F_{i_{1} i_{2} \ldots j} \omega_{j i_{r}}
$$

onde $F_{i_{1} i_{2} \ldots i_{r}}$ indicam as componentes de $F$ no referencial ortonormal $\left\{e_{1}, \ldots, e_{n}\right\}$.

É necessário mostrar que a definição anterior faz sentido, ou seja, que não depende da escolha de um referencial ortonormal $\left\{e_{1}, \ldots, e_{n}\right\}$.

Lema 1.5.3 Sejam F um tensor de ordem $r$ em uma variedade Riemanniana $M^{n}, p \in M^{n} e$ $\left\{e_{1}, \ldots, e_{n}\right\}$ um referencial ortonormal em uma vizinhança $U$ de $p$. Então a diferencial covariante $\nabla F$ não depende da escolha do referencial ortonormal $\left\{e_{1}, \ldots, e_{n}\right\}$. Além disso, a expressão da diferencial covariante $\nabla F$ para os campos de vetores $X_{1}, X_{2}, \ldots, X_{r}, Y \in \mathcal{X}(U)$ vale

$$
\begin{aligned}
\nabla F\left(X_{1}, X_{2}, \ldots, X_{r}, Y\right)= & Y\left(F\left(X_{1}, X_{2}, \ldots, X_{r}\right)\right)-F\left(\nabla_{Y} X_{1}, X_{2}, \ldots, X_{r}\right) \\
& -F\left(X_{1}, \nabla_{Y} X_{2}, \ldots, X_{r}\right)-\cdots-F\left(X_{1}, X_{2}, \ldots, \nabla_{Y} X_{r}\right) .
\end{aligned}
$$

Demonstração: Utilizando (1.10), observamos que

$$
\sum_{j=1}^{n} F_{i_{1} i_{2} \ldots i_{m-1} j i_{m+1} \ldots i_{r}} \omega_{j i_{m}}\left(e_{i}\right)=\sum_{j=1}^{n} F\left(e_{i_{1}}, e_{i_{2}}, \ldots, e_{i_{m-1}}, e_{j}, e_{i_{m+1}}, \ldots, e_{i_{r}}\right)\left\langle\nabla_{e_{i}} e_{j}, e_{i_{m}}\right\rangle .
$$

Pela propriedade (d) da Proposição 1.3.6, temos

$$
\left\langle\nabla_{e_{i}} e_{j}, e_{i_{m}}\right\rangle+\left\langle e_{j}, \nabla_{e_{i}} e_{i_{m}}\right\rangle=e_{i}\left(\left\langle e_{j}, e_{i_{m}}\right\rangle\right)=e_{i}\left(\delta_{j i_{m}}\right)=0,
$$

ou seja,

$$
\left\langle\nabla_{e_{i}} e_{j}, e_{i_{m}}\right\rangle=-\left\langle e_{j}, \nabla_{e_{i}} e_{i_{m}}\right\rangle .
$$

Por (1.16) e pelo fato de $F$ ser linear, temos

$$
\begin{aligned}
\sum_{j=1}^{n} F_{i_{1} i_{2} \ldots i_{m-1} j i_{m+1} \ldots i_{r}} \omega_{j i_{m}}\left(e_{i}\right) & =-\sum_{j=1}^{n} F\left(e_{i_{1}}, e_{i_{2}}, \ldots, e_{i_{m-1}}, e_{j}, e_{i_{m+1}}, \ldots, e_{i_{r}}\right)\left\langle e_{j}, \nabla_{e_{i}} e_{i_{m}}\right\rangle \\
& =-F\left(e_{i_{1}}, e_{i_{2}}, \ldots, e_{i_{m-1}}, \sum_{j=1}^{n}\left\langle\nabla_{e_{i}} e_{i_{m}}, e_{j}\right\rangle e_{j}, e_{i_{m+1}}, \ldots, e_{i_{r}}\right) \\
& =-F\left(e_{i_{1}}, e_{i_{2}}, \ldots, e_{i_{m-1}}, \nabla_{e_{i}} e_{i_{m}}, e_{i_{m+1}}, \ldots, e_{i_{r}}\right) .
\end{aligned}
$$

Portanto, 


$$
\begin{aligned}
\nabla F\left(e_{i_{1}}, e_{i_{2}}, \ldots, e_{i_{r}}, e_{i}\right)= & F_{i_{1} i_{2} \ldots i_{r} ; i}=\sum_{j=1}^{n} F_{i_{1} i_{2} \ldots i_{r} ; j} \omega_{j}\left(e_{i}\right) \\
= & \left(d F\left(e_{i_{1}}, e_{i_{2}}, \ldots, e_{i_{r}}\right)\right)\left(e_{i}\right)-F\left(\nabla_{e_{i}} e_{i_{1}}, e_{i_{2}}, \ldots, e_{i_{r}}\right) \\
& -F\left(e_{i_{1}}, \nabla_{e_{i}} e_{i_{2}}, \ldots, e_{i_{r}}\right)-\cdots-F\left(e_{i_{1}}, e_{i_{2}}, \ldots, \nabla_{e_{i}} e_{i_{r}}\right) .
\end{aligned}
$$

Sejam agora $X_{1}=\sum_{i_{1}=1}^{n} x_{i_{1}}^{1} e_{i_{1}}, \ldots, X_{r}=\sum_{i_{r}=1}^{n} x_{i_{r}}^{r} e_{i_{r}}, Y=\sum_{i=1}^{n} y_{i} e_{i}, r+1$ campos diferenciáveis de vetores em $U$. Usando a linearidade, a regra de derivação do produto e a expressão (1.17), temos

$$
\begin{aligned}
\nabla F\left(X_{1}, \ldots, X_{r}, Y\right)= & \nabla F\left(\sum_{i_{1}=1}^{n} x_{i_{1}}^{1} e_{i_{1}}, \ldots, \sum_{i_{r}=1}^{n} x_{i_{r}}^{r} e_{i_{r}}, \sum_{i=1}^{n} y_{i} e_{i}\right) \\
= & \sum_{i_{1}, \ldots, i_{r}, i=1}^{n} x_{i_{1}}^{1} \ldots x_{i_{r}}^{r} y_{i} \nabla F\left(e_{i_{1}}, \ldots, e_{i_{r}}, e_{i}\right) \\
= & \sum_{i_{1}, \ldots, i_{r}, i=1}^{n} x_{i_{1}}^{1} \ldots x_{i_{r}}^{r} y_{i}\left(\left(d F\left(e_{i_{1}}, \ldots, e_{i_{r}}\right)\right)\left(e_{i}\right)\right. \\
& \left.-F\left(\nabla_{e_{i}} e_{i_{1}}, \ldots, e_{i_{r}}\right)-\ldots-F\left(e_{i_{1}}, \ldots, \nabla_{e_{i}} e_{i_{r}}\right)\right) \\
= & \sum_{i_{1}, \ldots, i_{r}=1}^{n} x_{i_{1}}^{1} \ldots x_{i_{r}}^{r}\left(Y\left(F\left(e_{i_{1}}, \ldots, e_{i_{r}}\right)\right)\right. \\
& \left.-F\left(\nabla_{Y} e_{i_{1}}, \ldots, e_{i_{r}}\right)-\ldots-F\left(e_{i_{1}}, \ldots, \nabla_{Y} e_{i_{r}}\right)\right) \\
= & Y\left(F\left(X_{1}, \ldots, X_{r}\right)\right)+\sum_{i_{1}, \ldots, i_{r}=1}^{n} Y\left(x_{i_{1}}^{1} \ldots x_{i_{r}}^{r}\right) F\left(e_{i_{1}}, \ldots, e_{i_{r}}\right) \\
& -F\left(\nabla_{Y} X_{1}, \ldots, X_{r}\right)-\sum_{i_{1}=1}^{n} Y\left(x_{i_{1}}^{1}\right) F\left(e_{i_{1}}, \ldots, X_{r}\right) \\
& -\ldots-F\left(X_{1}, \ldots, \nabla_{Y} X_{r}\right)-\sum_{i_{r}=1}^{n} Y\left(x_{i_{r}}^{r}\right) F\left(X_{1}, \ldots, e_{i_{r}}\right) \\
= & Y\left(F\left(X_{1}, \ldots, X_{r}\right)\right)-F\left(\nabla_{Y} X_{1}, \ldots, X_{r}\right)-\ldots-F\left(X_{1}, \ldots, \nabla_{Y} X_{r}\right),
\end{aligned}
$$

o que demonstra (1.15) e mostra que a definição de $\nabla F$ não depende do referencial ortonormal.

Por fim, define-se o conceito de derivada covariante de tensores utilizando a noção de diferencial, de forma natural.

Definição 1.5.4 A derivada covariante de um tensor $F$ de ordem $r$, em relação a um campo diferenciável de vetores $X$, é o tensor $\nabla_{X} F$ de mesma ordem que $F$ dado por

$$
\nabla_{X} F\left(X_{1}, X_{2}, \ldots, X_{r}\right)=\nabla F\left(X_{1}, X_{2}, \ldots, X_{r}, X\right) .
$$

\subsection{Curvaturas}

Nesta seção serão discutidas algumas curvaturas que derivam das formas de curvatura $\Omega_{i j}$, apresentadas na Definição 1.2.4.

\subsubsection{Operador de Curvatura}

Definição 1.6.1.1 (Operador de Curvatura) Decorre do Lema 1.2.7 que, fixado $p \in M^{n}$, quando mudamos o referencial ortonormal $\left\{e_{1}, \ldots, e_{n}\right\}$, a matriz de formas $\left(\left(\Omega_{i j}\right)_{p}\right)$ muda como uma 
matriz de uma transformação linear em $T_{p} M$. Portanto, fixados dois vetores $X, Y \in T_{p} M$, a matriz $\left\{\left(\Omega_{i j}\right)_{p}(X, Y)\right\}$ é a matriz de uma aplicação linear

$$
\left(R_{X Y}\right)_{p}: T_{p} M \rightarrow T_{p} M
$$

$R_{X Y}$ é chamado operador de curvatura de $M^{n}$. Como $\Omega_{i j}$ é bilinear alternada e antissimétrica, temos que $(R)_{p}: T_{p} M \times T_{p} M \times T_{p} M \rightarrow T_{p} M$ é uma aplicação trilinear, e fixados $X, Y, Z \in T_{p} M$, $\operatorname{com} X=\sum_{i=1}^{n} x_{i} e_{i}, Y=\sum_{i=1}^{n} y_{i} e_{i}, Z=\sum_{i=1}^{n} z_{i} e_{i}$, vale:

$$
R_{X Y} Z=\left.\left(\begin{array}{cccc}
\Omega_{11} & \Omega_{12} & \ldots & \Omega_{1 n} \\
\Omega_{21} & \Omega_{22} & \ldots & \Omega_{2 n} \\
\vdots & \vdots & \ddots & \vdots \\
\Omega_{n 1} & \Omega_{n 2} & \ldots & \Omega_{n n}
\end{array}\right)\right|_{(X, Y)}\left(\begin{array}{c}
z_{1} \\
z_{2} \\
\vdots \\
z_{n}
\end{array}\right)=\sum_{i, j=1}^{n} \Omega_{i j}(X, Y) z_{j} e_{i} .
$$

Proposição 1.6.1.2 (Identidades do Operador de Curvatura) Sejam $M^{n}$ um variedade Riemanniana e $X, Y, Z, T$ campos diferenciáveis de vetores em $M^{n}$. Então:

(a) $\left\langle R_{X Y} Z, T\right\rangle=-\left\langle R_{Y X} Z, T\right\rangle$

(b) $\left\langle R_{X Y} Z, T\right\rangle=-\left\langle R_{X Y} T, Z\right\rangle$

(c) $\left\langle R_{X Y} Z, T\right\rangle=\left\langle R_{Z T} X, Y\right\rangle$

(d) $\left\langle R_{X Y} Z, T\right\rangle+\left\langle R_{Y Z} X, T\right\rangle+\left\langle R_{Z X} Y, T\right\rangle=0$ (Primeira Identidade de Bianchi)

Demonstração: Seja $\left\{e_{1}, \ldots, e_{n}\right\}$ um referencial ortonormal de $M^{n}$. Desta forma, podemos escrever $X=\sum_{i=1}^{n} x_{i} e_{i}, Y=\sum_{i=1}^{n} y_{i} e_{i}, Z=\sum_{i=1}^{n} z_{i} e_{i}, T=\sum_{i=1}^{n} t_{i} e_{i}$. Como $\Omega_{i j}=-\Omega j i$ e $\Omega_{i j}$ é uma forma bilinear alternada, segue:

$$
\left\langle R_{X Y} Z, T\right\rangle=\left\langle\sum_{i, j=1}^{n} \Omega_{i j}(X, Y) z_{j} e_{i}, T\right\rangle=-\left\langle\sum_{i, j=1}^{n} \Omega_{i j}(Y, X) z_{j} e_{i}, T\right\rangle=-\left\langle R_{Y X} Z, T\right\rangle,
$$

o que demonstra (a). Vale também:

$$
\begin{aligned}
\left\langle R_{X Y} Z, T\right\rangle & =\left\langle\sum_{i, j=1}^{n} \Omega_{i j}(X, Y) z_{j} e_{i}, \sum_{i=1}^{n} t_{i} e_{i}\right\rangle=\sum_{i, j=1}^{n} \Omega_{i j}(X, Y) z_{j} t_{i}=-\sum_{i, j=1}^{n} \Omega_{j i}(X, Y) t_{i} z_{j} \\
& \stackrel{i=l}{=}=k \\
& =\sum_{k, l=1}^{n} \Omega_{k l}(X, Y) t_{l} z_{k}=-\left\langle\sum_{k, l=1}^{n} \Omega_{k l}(X, Y) t_{l} e_{k}, \sum_{k=1}^{n} z_{k} e_{k}\right\rangle=-\left\langle R_{X Y} T, Z\right\rangle,
\end{aligned}
$$

o que demonstra (b). Derivando exteriormente (1.6), temos:

$$
\begin{aligned}
0 & =\sum_{i=1}^{n} d \omega_{j i} \wedge \omega_{i}-\sum_{k=1}^{n} \omega_{j k} \wedge d \omega_{k}=\sum_{i=1}^{n} d \omega_{j i} \wedge \omega_{i}-\sum_{k, i=1}^{n} \omega_{j k} \wedge \omega_{k i} \wedge \omega_{i} \\
& =\sum_{i=1}^{n}\left(d \omega_{j i}-\sum_{k=1}^{n} \omega_{j k} \wedge \omega_{k i}\right) \wedge \omega_{i}=\sum_{i=1}^{n} \Omega_{j i} \wedge \omega_{i}=-\sum_{i=1}^{n} \omega_{i} \wedge \Omega_{j i},
\end{aligned}
$$

ou seja,

$$
\sum_{i=1}^{n} \omega_{i} \wedge \Omega_{j i}=0
$$


Para cada $j=1, \ldots, n$, utilizando (1.18), podemos escrever:

$$
\begin{aligned}
0 & =\sum_{i=1}^{n} \omega_{i} \wedge \Omega_{j i}(X, Y, Z)=\sum_{i=1}^{n}\left(\omega_{i}(X) \Omega_{j i}(Y, Z)-\omega_{i}(Y) \Omega_{j i}(X, Z)+\omega_{i}(Z) \Omega_{j i}(X, Y)\right) \\
& =\sum_{i=1}^{n}\left(\Omega_{j i}(Y, Z) x_{i}-\Omega_{j i}(X, Z) y_{i}+\Omega_{j i}(X, Y) z_{i}\right)=\left\langle R_{Y Z} X-R_{X Z} Y+R_{X Y} Z, e_{j}\right\rangle
\end{aligned}
$$

donde

$$
R_{X Y} Z+R_{Y Z} X-R_{X Z} Y=0 .
$$

Logo, utilizando (a), segue

$$
\left\langle R_{X Y} Z, T\right\rangle+\left\langle R_{Y Z} X, T\right\rangle+\left\langle R_{Z X} Y, T\right\rangle=0,
$$

o que demonstra (d). Utilizando esta última identidade obtida, podemos escrever

$$
\left\{\begin{array}{l}
\left\langle R_{T Y} Z, X\right\rangle+\left\langle R_{Y Z} T, X\right\rangle+\left\langle R_{Z T} Y, X\right\rangle=0 \\
\left\langle R_{Y Z} X, T\right\rangle+\left\langle R_{Z X} Y, T\right\rangle+\left\langle R_{X Y} Z, T\right\rangle=0 \\
\left\langle R_{Z X} T, Y\right\rangle+\left\langle R_{X T} Z, Y\right\rangle+\left\langle R_{T Z} X, Y\right\rangle=0 \\
\left\langle R_{X T} Y, Z\right\rangle+\left\langle R_{T Y} X, Z\right\rangle+\left\langle R_{Y X} T, Z\right\rangle=0
\end{array}\right.
$$

Somando as equações acima e utilizando (a) e (b), concluímos que

$$
2\left\langle R_{Z T} Y, X\right\rangle+2\left\langle R_{X Y} Z, T\right\rangle=0 .
$$

Logo,

$$
\left\langle R_{X Y} Z, T\right\rangle=\left\langle R_{Z T} X, Y\right\rangle,
$$

o que demonstra (c).

Um exemplo de tensor em uma variedade Riemanniana é o seu tensor curvatura apresentado na próxima definição.

Definição 1.6.1.3 (Tensor Curvatura) $O$ tensor curvatura $R$ em $M^{n}$ é um tensor de ordem quatro, que faz corresponder a cada $p \in M^{n}$ e a cada conjunto de quatro vetores $X, Y, Z, T \in T_{p} M$, o valor

$$
R_{p}(X, Y, Z, T)=\left\langle R_{X Y} Z, T\right\rangle .
$$

Proposição 1.6.1.4 (Componentes do Tensor de Curvatura) Como as $\Omega_{i j}$ são 2-formas, elas podem ser escritas como

$$
\Omega_{i j}=-\frac{1}{2} \sum_{k, l=1}^{n} R_{i j k l} \omega_{k} \wedge \omega_{l}=-\sum_{\substack{k, l=1 \\ k<l}}^{n} R_{i j k l} \omega_{k} \wedge \omega_{l} .
$$

As funções $R_{i j k l}$ são chamadas de componentes do tensor de curvatura de $M^{n}$. É importante notar que vale

$$
R\left(e_{i}, e_{j}, e_{k}, e_{l}\right)=\left\langle R_{e_{i} e_{j}} e_{k}, e_{l}\right\rangle=R_{i j k l} .
$$

Demonstração: Utilizando as identidades presentes na Proposição 1.6.1.2, temos

$$
\left\langle R_{e_{i} e_{j}} e_{k}, e_{l}\right\rangle=\left\langle R_{e_{k} e_{l}} e_{i}, e_{j}\right\rangle=-\left\langle R_{e_{k} e_{l}} e_{j}, e i\right\rangle=-\left\langle\sum_{s=1}^{n} \Omega_{s j}\left(e_{k}, e_{l}\right) e_{s}, e_{i}\right\rangle
$$




$$
=-\Omega_{i j}\left(e_{k}, e_{l}\right)=\sum_{\substack{s, t=1 \\ s<t}}^{n} R_{i j s t} \omega_{s} \wedge \omega_{t}\left(e_{k}, e_{l}\right)=R_{i j k l}
$$

o que demonstra (1.19).

\subsubsection{Curvatura Seccional}

Munido do operador de curvatura apresentado anteriormente, é possível introduzir um dos tipos mais importantes de curvatura, a seccional.

Definição 1.6.2.1 Sejam $M^{n}$ uma variedade Riemanniana, $\sigma \subset T_{p} M$ um subespaço de dimensão 2 do espaço tangente $T_{p} M$ em $p \in M^{n}$ e $\left\{e_{1}, \ldots, e_{n}\right\}$ um referencial ortonormal em uma vizinhança de $p$ de tal modo que $e_{1}, e_{2}$ gerem $\sigma$. O número

$$
K_{p}(\sigma)=-\left(\Omega_{12}\right)_{p}\left(e_{1}, e_{2}\right)=\left\langle\left(R_{e_{1} e_{2}}\right)_{p}\left(e_{1}\right), e_{2}\right\rangle
$$

é chamado a curvatura seccional de $M^{n}$ em $p$, segundo $\sigma$.

O próximo lema mostra que o número $\left(\Omega_{12}\right)_{p}\left(e_{1}, e_{2}\right)$ depende apenas do subespaço $\sigma$.

Lema 1.6.2.2 $A$ curvatura seccional de $M^{n}$ em $p$ segundo $\sigma\left(K_{p}(\sigma)\right)$ depende apenas do subespaço $\sigma$. Além disso, dados $X, Y \in \sigma \subset T_{p} M$ dois vetores linearmente independentes quaisquer, a expressão da curvatura seccional em termos do operador de curvatura é dada por

$$
K(\sigma)=\frac{\left\langle R_{X Y} X, Y\right\rangle}{\|X \wedge Y\|^{2}}
$$

onde $\|X \wedge Y\|=\sqrt{\|X\|^{2}\|Y\|^{2}-\langle X, Y\rangle^{2}}$ representa a área do paralelogramo bidimensional determinado pelo par de vetores $X, Y$.

Demonstração: Seja $\left\{\bar{e}_{1}, \ldots, \bar{e}_{n}\right\}$ um outro referencial ortonormal em torno de $p$ de modo que $\bar{e}_{1}$, $\bar{e}_{2}$ ainda gerem $\sigma$. Então $e_{i}=\sum_{j=1}^{n} u_{i j} \bar{e}_{j}$, onde a matriz $U=\left(u_{i j}\right)$ é da forma $U=\left(\begin{array}{c|c}A & 0 \\ \hline 0 & B\end{array}\right)$, com $A=\left(\begin{array}{cc}\cos \theta & \operatorname{sen} \theta \\ -\operatorname{sen} \theta & \cos \theta\end{array}\right)$ ou $A=\left(\begin{array}{cc}-\operatorname{sen} \theta & \cos \theta \\ \cos \theta & \operatorname{sen} \theta\end{array}\right)$ dependendo da orientação de $\bar{e}_{1}, \bar{e}_{2}$ relativamente a $e_{1}, e_{2}$. Pelo Lema 1.2.7,

$$
\Omega_{i j}=\sum_{k, l=1}^{n} u_{i k} \bar{\Omega}_{k l} u_{j l}
$$

Logo,

$$
\Omega_{12}=\sum_{k, l=1}^{n} u_{1 k} u_{2 l} \bar{\Omega}_{k l}= \pm\left(\cos ^{2} \theta \bar{\Omega}_{12}-\operatorname{sen}^{2} \theta \bar{\Omega}_{21}\right)= \pm \bar{\Omega}_{12},
$$

onde o sinal depende da orientação. Portanto,

$$
\Omega_{12}\left(e_{1}, e_{2}\right)= \pm \bar{\Omega}_{12}\left(e_{1}, e_{2}\right)=\bar{\Omega}_{12}\left(\bar{e}_{1}, \bar{e}_{2}\right)
$$

qualquer que seja a orientação adotada, provando que $K_{p}(\sigma)$ depende apenas dos subespaço $\sigma$. Como $X, Y \in \sigma \subset T_{p} M$, então $X=x_{1} e_{1}+x_{2} e_{2}, Y=y_{1} e_{1}+y_{2} e_{2}$. Por linearidade e pelas identidades presentes na Proposição 1.6.1.2,

$$
\begin{aligned}
\left\langle R_{X Y} X, Y\right\rangle & =\left\langle R_{x_{1} e_{1}+x_{2} e_{2}, y_{1} e_{1}+y_{2} e_{2}} X, Y\right\rangle \\
& =x_{1} y_{1}\left\langle R_{e_{1} e_{1}} X, Y\right\rangle+x_{1} y_{2}\left\langle R_{e_{1} e_{2}} X, Y\right\rangle+x_{2} y_{1}\left\langle R_{e_{2} e_{1}} X, Y\right\rangle+x_{2} y_{2}\left\langle R_{e_{2} e_{2}} X, Y\right\rangle \\
& =\left(x_{1} y_{2}-x_{2} y_{1}\right)\left\langle R_{e_{1} e_{2}} X, Y\right\rangle=\left(x_{1} y_{2}-x_{2} y_{1}\right)\left\langle R_{e_{1} e_{2}}\left(x_{1} e_{1}+x_{2} e_{2}\right), y_{1} e_{1}+y_{2} e_{2}\right\rangle
\end{aligned}
$$




$$
\begin{aligned}
= & \left(x_{1} y_{2}-x_{2} y_{1}\right)\left(x_{1} y_{1}\left\langle R_{e_{1} e_{2}} e_{1}, e_{1}\right\rangle+x_{1} y_{2}\left\langle R_{e_{1} e_{2}} e_{1}, e_{2}\right\rangle+x_{2} y_{1}\left\langle R_{e_{1} e_{2}} e_{2}, e_{1}\right\rangle\right. \\
& \left.+x_{2} y_{2}\left\langle R_{e_{1} e_{2}} e_{2}, e_{2}\right\rangle\right)=\left(x_{1} y_{2}-x_{2} y_{1}\right)^{2}\left\langle R_{e_{1} e_{2}} e_{1}, e_{2}\right\rangle=\|X \wedge Y\|^{2} K(\sigma),
\end{aligned}
$$

o que demonstra (1.20).

Uma das importâncias da curvatura seccional é que o conhecimento de $K(\sigma)$, para todo $\sigma$, determina completamente a curvatura $R$.

Lema 1.6.2.3 Seja $V$ um espaço vetorial de dimensão $\geq 2$, munido de um produto interno $\langle$,$\rangle .$ Sejam $R: V \times V \times V \rightarrow V$ e $R^{\prime}: V \times V \times V \rightarrow V$ aplicações trilineares tais que as identidades (a), (b), (c) e (d) da Proposição 1.6.1.2 sejam satisfeitas para $\left\langle R_{X Y} Z, T\right\rangle$ e $\left\langle R_{X Y}^{\prime} Z, T\right\rangle$. Se $X, Y$ são dois vetores linearmente independentes quaisquer, escrevamos,

$$
K(\sigma)=\frac{\left\langle R_{X Y} X, Y\right\rangle}{\|X \wedge Y\|^{2}}, \quad K^{\prime}(\sigma)=\frac{\left\langle R_{X Y}^{\prime} X, Y\right\rangle}{\|X \wedge Y\|^{2}},
$$

onde $\sigma$ é o subespaço bidimensional gerado por $X$ e $Y$. Se para todo $\sigma \subset V, K(\sigma)=K^{\prime}(\sigma)$, então $R=R^{\prime}$.

Demonstração: Basta provar que $\left\langle R_{X Y} Z, T\right\rangle=\left\langle R_{X Y}^{\prime} Z, T\right\rangle$ para quaisquer $X, Y, Z, T \in V$. Por hipótese, temos que $\left\langle R_{X Y} X, Y\right\rangle=\left\langle R_{X Y}^{\prime} X, Y\right\rangle, \forall X, Y \in V$. Então

$$
\left\langle R_{X+Z, Y} X+Z, Y\right\rangle=\left\langle R_{X+Z, Y}^{\prime} X+Z, Y\right\rangle .
$$

Daí,

$$
\left\langle R_{X Y} X, Y\right\rangle+2\left\langle R_{X Y} Z, Y\right\rangle+\left\langle R_{Z Y} Z, Y\right\rangle=\left\langle R_{X Y}^{\prime} X, Y\right\rangle+2\left\langle R_{X Y}^{\prime} Z, Y\right\rangle+\left\langle R_{Z Y}^{\prime} Z, Y\right\rangle
$$

ou seja,

$$
\left\langle R_{X Y} Z, Y\right\rangle=\left\langle R_{X Y}^{\prime} Z, Y\right\rangle,
$$

para todo $X, Y, Z \in V$. Com este resultado, temos

$$
\left\langle R_{X, Y+T} Z, Y+T\right\rangle=\left\langle R_{X, Y+T}^{\prime} Z, Y+T\right\rangle .
$$

Desenvolvendo obtemos

$$
\begin{aligned}
\left\langle R_{X Y} Z, Y\right\rangle+ & \left\langle R_{X Y} Z, T\right\rangle+\left\langle R_{X T} Z, Y\right\rangle+\left\langle R_{X T} Z, T\right\rangle=\left\langle R_{X Y}^{\prime} Z, Y\right\rangle \\
& +\left\langle R_{X Y}^{\prime} Z, T\right\rangle+\left\langle R_{X T}^{\prime} Z, Y\right\rangle+\left\langle R_{X T}^{\prime} Z, T\right\rangle
\end{aligned}
$$

ou seja,

$$
\left\langle R_{X Y} Z, T\right\rangle+\left\langle R_{X T} Z, Y\right\rangle=\left\langle R_{X Y}^{\prime} Z, T\right\rangle+\left\langle R_{X T}^{\prime} Z, Y\right\rangle .
$$

Rearranjando os termos chegamos a

$$
\left\langle R_{X Y} Z, T\right\rangle-\left\langle R_{X Y}^{\prime} Z, T\right\rangle=\left\langle R_{Y Z} X, T\right\rangle-\left\langle R_{Y Z}^{\prime} X, T\right\rangle .
$$

Isto nos leva a concluir que a expressão $\left\langle R_{X Y} Z, T\right\rangle-\left\langle R_{X Y}^{\prime} Z, T\right\rangle$ é invariante por permutações cíclicas dos primeiros três elementos. Portanto, por (d) da Proposição 1.6.1.2, temos

$$
3\left(\left\langle R_{X Y} Z, T\right\rangle-\left\langle R_{X Y}^{\prime} Z, T\right\rangle\right)=0 .
$$

Logo,

$$
\left\langle R_{X Y} Z, T\right\rangle=\left\langle R_{X Y}^{\prime} Z, T\right\rangle,
$$

para todo $X, Y, Z, T \in V$.

Este trabalho trata de imersões em variedades Riemannianas com curvatura constante e o lema 
a seguir permite obter uma caracterização de tais variedades, utilizando as componentes $R_{i j k l}$ do operador de curvatura.

Lema 1.6.2.4 Sejam $M$ uma variedade Riemanniana e $p$ um ponto de $M$. Defina uma aplicação trilinear $R^{\prime}: T_{p} M \times T_{p} M \times T_{p} M \rightarrow T_{p} M$ por

$$
\left\langle R_{X Y}^{\prime} Z, T\right\rangle=\langle X, Z\rangle\langle Y, T\rangle-\langle Y, Z\rangle\langle X, T\rangle, \quad \forall X, Y, Z, T \in T_{p} M .
$$

Então $M$ tem curvatura seccional constante igual a c se e só se $R=c R^{\prime}$, onde $R$ é a curvatura de $M$.

Demonstração: Primeiro verificamos que $\left\langle R_{X Y}^{\prime} Z, T\right\rangle$ satisfaz as identidades da Proposição 1.6.1.2. De fato,

$$
\begin{aligned}
\left\langle R_{X Y}^{\prime} Z, T\right\rangle & =\langle X, Z\rangle\langle Y, T\rangle-\langle Y, Z\rangle\langle X, T\rangle \\
& =-(\langle Y, Z\rangle\langle X, T\rangle-\langle X, Z\rangle\langle Y, T\rangle) \\
& =-\left\langle R_{Y X}^{\prime} Z, T\right\rangle .
\end{aligned}
$$

$$
\begin{aligned}
\left\langle R_{X Y}^{\prime} Z, T\right\rangle & =\langle X, Z\rangle\langle Y, T\rangle-\langle Y, Z\rangle\langle X, T\rangle \\
& =-(\langle X, T\rangle\langle Y, Z\rangle-\langle Y, T\rangle\langle X, Z\rangle) \\
& =-\left\langle R^{\prime}{ }_{X Y} T, Z\right\rangle .
\end{aligned}
$$

(c)

$$
\begin{aligned}
\left\langle R_{X Y}^{\prime} Z, T\right\rangle & =\langle X, Z\rangle\langle Y, T\rangle-\langle Y, Z\rangle\langle X, T\rangle \\
& =\langle Z, X\rangle\langle T, Y\rangle-\langle T, X\rangle\langle Z, Y\rangle \\
& =\left\langle R^{\prime}{ }_{Z T} X, Y\right\rangle .
\end{aligned}
$$

$$
\begin{aligned}
\left\langle R_{X Y}^{\prime} Z, T\right\rangle+\left\langle R_{Y Z}^{\prime} X, T\right\rangle+ & \left\langle R_{Z X}^{\prime} Y, T\right\rangle \\
= & \langle X, Z\rangle\langle Y, T\rangle-\langle Y, Z\rangle\langle X, T\rangle \\
& +\langle Y, X\rangle\langle Z, T\rangle-\langle Z, X\rangle\langle Y, T\rangle \\
& +\langle Z, Y\rangle\langle X, T\rangle-\langle X, Y\rangle\langle Z, T\rangle \\
= & 0 .
\end{aligned}
$$

Admita que $K_{p}(\sigma)=c, \forall \sigma \subset T_{p} M$. Como

$$
\left\langle R_{X Y}^{\prime} X, Y\right\rangle=\langle X, X\rangle\langle Y, Y\rangle-\langle X, Y\rangle^{2}=\|X \wedge Y\|^{2},
$$

temos que, para todo par de vetores $X, Y \in T_{p} M$ linearmente independentes,

$$
\left\langle R_{X Y} X, Y\right\rangle=c\|X \wedge Y\|^{2}=c\left\langle R_{X Y}^{\prime} X, Y\right\rangle .
$$

Pelo Lema 1.6.2.3, isto implica que, para todo $X, Y, Z, T \in T_{p} M$,

$$
\left\langle R_{X Y} Z, T\right\rangle=c\left\langle R_{X Y}^{\prime} Z, T\right\rangle,
$$

donde $R=c R^{\prime}$. A recíproca é imediata.

Corolário 1.6.2.5 Sejam $M^{n}$ uma variedade Riemanniana, $p$ um ponto de $M^{n}$ e $\left\{e_{1}, \ldots, e_{n}\right\}$ uma base ortonormal de $T_{p} M$. Então $K_{p}(\sigma)=c$ para todo $\sigma \subset T_{p} M$ se, e somente se,

$$
R_{i j k l}=c\left(\delta_{i k} \delta_{j l}-\delta_{i l} \delta_{j k}\right),
$$

onde $\delta_{i j}$ é o delta de Kronecker. 


\subsubsection{Curvaturas de Ricci e Escalar}

Como algumas combinações das curvaturas seccionais aparecem frequentemente, elas recebem nomes próprios e algumas serão apresentadas a seguir.

Definição 1.6.3.1 (Tensor de Ricci) Sejam $M^{n}$ uma variedade Riemanniana, $p \in M^{n} e$ $\left\{e_{1}, \ldots, e_{n}\right\}$ um referencial ortonormal. Para cada par de vetores $x, y \in T_{p} M$ definimos

$$
Q(x, y)=\text { traço da aplicação } z \rightarrow R(x, z) y=\sum_{i=1}^{n}\left\langle R_{x e_{i}} y, e_{i}\right\rangle .
$$

Observe que

$$
Q(x, y)=\sum_{i=1}^{n}\left\langle R_{x e_{i}} y, e_{i}\right\rangle=\sum_{i=1}^{n}\left\langle R_{y e_{i}} x, e_{i}\right\rangle=Q(y, x) .
$$

Logo, Q é bilinear e simétrica. Esta forma bilinear é chamada de tensor de Ricci, e seus coeficientes, em um referencial ortonormal $\left\{e_{1}, \ldots, e_{n}\right\}$, são dados por

$$
R_{i j}=Q\left(e_{i}, e_{j}\right)=\sum_{k=1}^{n}\left\langle R_{e_{i} e_{k}} e_{j}, e_{k}\right\rangle=\sum_{k=1}^{n} R_{i k j k} .
$$

Definição 1.6.3.2 (Curvatura de Ricci) Sejam $M^{n}$ uma variedade Riemanniana e $p \in M^{n}$. Dado $x \in T_{p} M$ unitário, o valor $\operatorname{Ric}_{p}(x)=Q(x, x)$ é chamado de curvatura de Ricci na direção $x$.

Definição 1.6.3.3 (Curvatura Escalar) Sejam $M^{n}$ uma variedade Riemanniana, $p \in M^{n} e$ $\left\{e_{1}, \ldots, e_{n}\right\}$ um referencial ortonormal. O valor $K(p)=\sum_{i=1}^{n} Q\left(e_{i}, e_{i}\right)$ é chamado de curvatura escalar em $p$. Como à forma bilinear $Q$ em $T_{p} M$ corresponde uma aplicação linear auto-adjunta $K$, dada por $\langle K(x), y\rangle=Q(x, y)$, temos

$$
\text { traço de } K=\sum_{i=1}^{n}\left\langle K\left(e_{i}\right), e_{i}\right\rangle=\sum_{i=1}^{n} Q\left(e_{i}, e_{i}\right)=K(p),
$$

ou seja, $K(p)$ não depende do referencial ortonormal escolhido.

\subsection{Operadores Diferenciais}

A partir da derivação covariante apresentada anteriormente, é possível estender às variedades Riemannianas certos operados diferenciais, bastante utilizados no $\mathbb{R}^{n}$.

Definição 1.7.1 (Operadores Diferenciais) Seja $f \in \mathcal{D}(M)$ uma função diferenciável em uma variedade Riemanniana $M^{n}$. O gradiente de $f$ é o campo vetorial grad $f$ em $M^{n}$ definido por

$$
\langle\operatorname{grad} f, X\rangle_{p}=d f_{p}(X), \quad \forall p \in M^{n}, \quad \forall X \in T_{p} M .
$$

Em outras palavras, grad $f$ é o dual, na métrica Riemanniana, da forma df. Considerando um referencial ortonormal $\left\{e_{1}, \ldots, e_{n}\right\}$ em um aberto $U \subset M^{n}$, podemos escrever, em $U, d f=\sum_{i=1}^{n} f_{i} \omega_{i}$, onde $f_{i}$ é chamada a derivada de $f$ na direção $e_{i}$. Com isso, em $U$,

$$
\operatorname{grad} f=\sum_{i=1}^{n} f_{i} e_{i}
$$

A diferencial covariante de df é dada por 


$$
\nabla(d f)=\sum_{i, j=1}^{n} f_{i ; j} \omega_{i} \omega_{j},
$$

onde, por (1.14),

$$
\sum_{j=1}^{n} f_{i ; j} \omega_{j}=d f_{i}+\sum_{j=1}^{n} f_{j} \omega_{j i} .
$$

A forma bilinear $\nabla(d f)$ ou Hess $f$ é chamado o Hessiano de $f$ na métrica de $M^{n}$. O traço desta forma bilinear, isto é, a função em $M^{n}$ dada por

$$
\Delta f=\sum_{i=1}^{n} f_{i ; i}
$$

é chamado o Laplaciano de f. Dado $X \in \mathcal{X}(M)$, a métrica Riemanniana de $M^{n}$ faz corresponder a $X$ uma 1-forma diferencial $\omega^{X}$ dada por

$$
\omega^{X}(Y)=\langle X, Y\rangle_{p}, \quad \forall p \in M, \quad \forall Y \in T_{p} M .
$$

Dado um referencial ortonormal local $\left\{e_{1}, \ldots, e_{n}\right\}$, se $X=\sum_{i=1}^{n} x_{i} e_{i}$, então

$$
\omega^{X}=\sum_{i=1}^{n} x_{i} \omega_{i}
$$

onde, por (1.14),

$$
\sum_{j=1}^{n} x_{i ; j} \omega_{j}=d x_{i}+\sum_{j=1}^{n} x_{j} \omega_{j i} .
$$

O traço de $\omega^{X}$, isto é, a função em $M^{n}$ dada por

$$
\operatorname{div} X=\sum_{i=1}^{n} x_{i ; i}
$$

é chamada a divergência de $X$, e vale

$$
\Delta f=\operatorname{div} \operatorname{grad} f .
$$

Lema 1.7.2 Sejam $M^{n}$ uma variedade Riemanniana, $U \subset M^{n}$ um aberto $e\left\{e_{1}, \ldots, e_{n}\right\}$ um referencial ortonormal de $U$. Se $f, g \in \mathcal{D}(U)$ são funções diferenciáveis em $U$ e $X \in \mathcal{X}(U)$, $X=\sum_{i=1}^{n} x_{i} e_{i}$, um campo de vetores diferenciáveis em $U$. Então valem

$$
\begin{gathered}
\operatorname{div}(f X)=f \operatorname{div} X+X(f), \\
\Delta(f g)=f \Delta g+g \Delta f+2\langle\operatorname{grad} f, \operatorname{grad} g\rangle .
\end{gathered}
$$

Demonstração: Observe que para $Z \in \mathcal{X}(U) \operatorname{com} Z=\sum_{i=1}^{n} z_{i} e_{i}$, por (1.24) vale

$$
\begin{aligned}
\operatorname{div} Z & =\sum_{i=1}^{n} z_{i ; i}=\sum_{i=1}^{n}\left(\sum_{j=1}^{n} z_{i ; j} \omega_{j}\left(e_{i}\right)\right) \\
& =\sum_{i=1}^{n}\left(d z_{i}\left(e_{i}\right)+\sum_{j=1}^{n} z_{j} \omega_{j i}(e i)\right) .
\end{aligned}
$$


Seja $Y \in \mathcal{X}(U)$ dado por

$$
Y=f X=\sum_{i=1}^{n} f x_{i} e_{i}=\sum_{i=1}^{n} y_{i} e_{i}
$$

Então vale

$$
\begin{aligned}
\operatorname{div}(f X)= & \operatorname{div} Y=\sum_{i=1}^{n}\left(d y_{i}\left(e_{i}\right)+\sum_{j=1}^{n} y_{j} \omega_{j i}(e i)\right)=\sum_{i=1}^{n}\left(d\left(f x_{i}\right)\left(e_{i}\right)+\sum_{j=1}^{n} f x_{j} \omega_{j i}(e i)\right) \\
= & \sum_{i=1}^{n}\left(x_{i} d f\left(e_{i}\right)+f d x_{i}\left(e_{i}\right)+f \sum_{j=1}^{n} x_{j} \omega_{j i}(e i)\right)=d f\left(\sum_{i=1}^{n} x_{i} e_{i}\right) \\
& +f \sum_{i=1}^{n}\left(d x_{i}\left(e_{i}\right)+\sum_{j=1}^{n} x_{j} \omega_{j i}(e i)\right)=d f(X)+f \operatorname{div} X=f \operatorname{div} X+X(f),
\end{aligned}
$$

o que demonstra (1.26). Seja $h \in \mathcal{D}(U)$ dada por $h=f g$, então

$$
d h=d(f g)=f d g+g d f=f \sum_{i=1}^{n} g_{i} \omega_{i}+g \sum_{i=1}^{n} f_{i} \omega_{i}=\sum_{i=1}^{n}\left(f g_{i}+g f_{i}\right) \omega_{i}=\sum_{i=1}^{n} h_{i} \omega_{i} .
$$

Logo, de (1.25) vale

$$
\begin{aligned}
\Delta(f g) & =\Delta h=\operatorname{div} \operatorname{grad} h=\operatorname{div}\left(\sum_{i=1}^{n} h_{i} e_{i}\right)=\sum_{i=1}^{n}\left(d h_{i}\left(e_{i}\right)+\sum_{j=1}^{n} h_{j} \omega_{j i}(e i)\right) \\
& =\sum_{i=1}^{n}\left(d\left(f g_{i}+g f_{i}\right)\left(e_{i}\right)+\sum_{j=1}^{n}\left(f g_{j}+g f_{j}\right) \omega_{j i}(e i)\right) \\
& =\sum_{i=1}^{n}\left(f d g_{i}\left(e_{i}\right)+g_{i} d f(e i)+g d f_{i}\left(e_{i}\right)+f_{i} d g\left(e_{i}\right)+f \sum_{j=1}^{n} g_{j} \omega_{j i}\left(e_{i}\right)+g \sum_{j=1}^{n} f_{j} \omega_{j i}\left(e_{i}\right)\right) \\
& =f \sum_{i=1}^{n}\left(d g_{i}\left(e_{i}\right)+\sum_{j=1}^{n} g_{j} \omega_{j i}(e i)\right)+g \sum_{i=1}^{n}\left(d f_{i}\left(e_{i}\right)+\sum_{j=1}^{n} f_{j} \omega_{j i}(e i)\right)+2 \sum_{i=1}^{n} f_{i} g_{i} \\
& =f \Delta g+g \Delta f+2\left\langle\sum_{i=1}^{n} f_{i} e_{i}, \sum_{i=1}^{n} g_{i} e_{i}\right\rangle=f \Delta g+g \Delta f+2\langle\operatorname{grad} f, \operatorname{grad} g\rangle,
\end{aligned}
$$

o que demonstra (1.27).

\subsection{Imersões Riemannianas}

Nesta seção serão apresentadas algumas definições e resultados, obtidos para imersões em variedades Riemannianas.

Definição 1.8.1 (Imersão Riemanniana) Sejam $M^{n}$ e $\bar{M}^{n+q}$ variedades Riemannianas $e$ $x: M^{n} \rightarrow \bar{M}^{n+q}$ uma imersão. Dizemos que $x$ é uma imersão Riemanniana (ou isométrica) se

$$
\left\langle v_{1}, v_{2}\right\rangle_{p}=\left\langle d x\left(v_{1}\right), d x\left(v_{2}\right)\right\rangle_{x(p)}, \quad \forall p \in M^{n}, \quad \forall v_{1}, v_{2} \in T_{p} M,
$$

ou seja, x é uma imersão Riemanniana se a métrica induzida coincide com a métrica original. 
Lema 1.8.2 (Referencial Ortonormal Adaptado) Sejam $M^{n}$ e $\bar{M}^{n+q}$ variedades Riemannianas e $x: M^{n} \rightarrow \bar{M}^{n+q}$ uma imersão Riemanniana. Então, para todo $p \in M^{n}$ existe uma vizinhança $U \subset M^{n}$ de $p$ e uma vizinhança $V \subset \bar{M}^{n+q}$ de $x(p)$ em $\bar{M}^{n+q}$ com $V \supset x(U)$, tal que existe um referencial ortonormal móvel $\left\{e_{1}, \ldots, e_{n}, e_{n+1}, \ldots, e_{n+q}\right\}$ em $V$ com a propriedade que, quando restritos a $x(U)$, os vetores $e_{1}, \ldots, e_{n}$ são tangentes a $x(U)$ e os vetores $e_{n+1}, \ldots, e_{n+q}$ são normais a $x(U)$. Um tal referencial ortonormal é chamado de referencial ortonormal adaptado a $x$.

Demonstração: Como uma consequência do Teorema da Função Inversa, temos a existência de $U \subset M^{n}$ e $V \subset \bar{M}^{n+q}$ com $V \supset x(U)$. Se $V$ é suficientemente pequeno, existe um difeomorfismo $g: V \rightarrow V$ tal que $g \circ x(U)$ é um aberto de uma variedade linear de dimensão $n$ em $\bar{M}^{n+q}$. Logo, existe um referencial ortonormal $\left\{f_{1}, \ldots, f_{n+q}\right\}$. A imagem inversa $\left\{d g^{-1}\left(f_{1}\right), \ldots, d g^{-1}\left(f_{n+q}\right)\right\}$ deste referencial ortonormal pode não ser ortonormal mas basta utilizar o processo de Gram-Schmidt em cada ponto de $V$, obtendo assim um referencial ortonormal adaptado a $x(U)$.

Definição 1.8.3 (Hipersuperfície) Sejam $M^{n}$ e $\bar{M}^{n+1}$ variedades Riemannianas e $x: M^{n} \rightarrow \bar{M}^{n+1}$ uma imersão isométrica. Nessas condições, $x\left(M^{n}\right) \subset \bar{M}^{n+1}$ recebe o nome de hipersuperfície.

A partir de agora utilizaremos uma usual convenção de índices. Sejam $M^{n}$ e $\bar{M}^{n+q}$ variedades Riemannianas e $x: M^{n} \rightarrow \bar{M}^{n+q}$ uma imersão isométrica. Dado um ponto $p \in M^{n}$, tomaremos uma vizinhança $U \subset M^{n}$ de $p$ de tal modo que $\left.x\right|_{U}$ seja injetiva, e uma vizinhança $V \subset \bar{M}^{n+q}$ de $x(p)$ tal que $V \supset x(U)$ de modo que em $V$ seja possível obter um referencial ortonormal $\left\{e_{1}, \ldots, e_{n+q}\right\}$, adaptado a $x$. Além disso, $U \subset M^{n}$ será identificado com $x(U) \subset \bar{M}^{n+q}$ e valerá os seguintes domínios de índices:

$$
\left\{\begin{array}{c}
1 \leq A, B, C, \ldots \leq n+q \\
1 \leq i, j, k, \ldots \leq n \\
n+1 \leq \alpha, \beta, \gamma, \ldots \leq n+q .
\end{array}\right.
$$

Definição 1.8.4 (Espaço Normal) $O$ espaço tangente $T_{p} \bar{M}$ de $\bar{M}$ em $p$ se decompõe em uma soma direta $T_{p} \bar{M}=T_{p} M \oplus N_{p} M$, onde identifica-se $d x_{p}\left(T_{p} M\right) \approx T_{p} M$ e denota-se por $N_{p} M o$ complemento ortogonal de $T_{p} M$ em $T_{p} \bar{M}$. $N_{p} M$ é chamado de espaço normal da imersão $x$ em $p$.

Definição 1.8.5 (Campo de Vetores Normal) Um campo de vetores normal $\nu$ é uma correspondência que a cada $p \in M^{n}$ associa um vetor $\nu(p) \in N_{p} M$, de tal modo que para todo referencial ortonormal adaptado $\left\{e_{1}, \ldots, e_{n+q}\right\}$ em uma vizinhança $V \subset \bar{M}^{n+q}$ de $p$, as funções $\nu_{\alpha}$ dadas por $\nu=\sum_{\alpha=n+1}^{n+q} \nu_{\alpha} e_{\alpha}$ sejam diferenciáveis em $p$. Note que tal condição não depende da escolha do referencial ortonormal.

Em $V$ existem formas $\omega_{A}, \omega_{A B}$ que satisfazem as equações de estrutura presentes no Teorema 1.2.5:

$$
\begin{gathered}
d \omega_{A}=\sum_{B=1}^{n+q} \omega_{A B} \wedge \omega_{B}=\sum_{B=1}^{n+q} \omega_{B} \wedge \omega_{B A} \\
d \omega_{A B}=\sum_{C=1}^{n+q} \omega_{A C} \wedge \omega_{C B}+\bar{\Omega}_{A B}, \quad \bar{\Omega}_{A B}=-\frac{1}{2} \sum_{C, D=1}^{n+q} \bar{R}_{A B C D} \omega_{C} \wedge \omega_{D} .
\end{gathered}
$$

Tais formas também valem para $U$, onde podemos escrever, restringindo a vetores tangentes:

$$
\begin{gathered}
d \omega_{i}=\sum_{j=1}^{n} \omega_{i j} \wedge \omega_{j}=\sum_{j=1}^{n} \omega_{j} \wedge \omega_{j i} \\
d \omega_{i j}=\sum_{k=1}^{n} \omega_{i k} \wedge \omega_{k j}+\Omega_{i j}, \quad \Omega_{i j}=-\frac{1}{2} \sum_{k, l=1}^{n} R_{i j k l} \omega_{k} \wedge \omega_{l} .
\end{gathered}
$$


As restrições destas formas em $U \subset V$ satisfazem as mesmas equações de estrutura e, como o referencial ortonormal é adaptado, $\omega_{\alpha}=0$. Logo,

$$
0=d \omega_{\alpha}=\sum_{i=1}^{n} \omega_{i} \wedge \omega_{i \alpha},
$$

e, pelo Lema 1.2.1,

$$
\omega_{i \alpha}=\sum_{j=1}^{n} h_{i j}^{\alpha} \omega_{j}, \quad h_{i j}^{\alpha}=h_{j i}^{\alpha} .
$$

No caso de uma hipersuperfície, o índice $\alpha$ não se faz necessário já que a única direção normal é $e_{n+1}, \operatorname{logo}$

$$
\omega_{i, n+1}=\sum_{j=1}^{n} h_{i j} \omega_{j}, \quad h_{i j}=h_{j i} .
$$

\subsubsection{Segunda Forma Fundamental}

Nesta seção será apresentada a segunda forma fundamental de uma imersão Riemanniana $x: M \rightarrow \bar{M}$. Uma das importâncias desta forma é relacionar as curvaturas de $M$ e $\bar{M}$, como será visto nas próximas seções.

Definição 1.8.1.1 (Segunda Forma Fundamental) Sejam $M^{n}$ e $\bar{M}^{n+q}$ variedades Riemannianas, $x: M^{n} \rightarrow \bar{M}^{n+q}$ uma imersão Riemanniana $e\left\{e_{1}, \ldots, e_{n}, e_{n+1}, \ldots, e_{n+q}\right\}$ um referencial ortonormal adaptado a $M^{n}$. A forma quadrática $h^{\alpha}=\sum_{i, j=1}^{n} h_{i j}^{\alpha} \omega_{i} \omega_{j}$ é a segunda forma fundamental de $x$ na direção $e_{\alpha}$.

Lema 1.8.1.2 Sejam $M^{n}$ e $\bar{M}^{n+q}$ variedades Riemannianas, $x: M^{n} \rightarrow \bar{M}^{n+q}$ uma imersão Riemanniana, $U \subset M^{n}$ um aberto e $\nu$ um campo unitário normal em $M^{n}$. Seja $\left\{e_{1}, \ldots, e_{n}, e_{n+1}, \ldots, e_{n+q}\right\}$ um referencial ortonormal adaptado a $U$ de modo que $e_{n+1}=\nu$. Então $h^{\nu}=h^{n+1}$, chamada de a segunda forma fundamental de $x$ na direção $\nu$, não depende da escolha do referencial ortonormal e está globalmente definida.

Demonstração: Sejam $p \in M^{n}$ e $U \subset M^{n}$ uma vizinhança de $p$. Dado $v \in T_{p} M$ qualquer, seja $\alpha:(-\epsilon, \epsilon) \rightarrow U$ uma curva parametrizada pelo comprimento de arco, com $\alpha(0)=p$ e $\alpha^{\prime}(0)=v$. Escolhendo um referencial ortonormal adaptado $\left\{e_{1}, \ldots, e_{n}, e_{n+1}, \ldots, e_{n+q}\right\} \operatorname{com} e_{1}=\alpha^{\prime}(s)$ e $e_{n+1}=\nu$, temos

$$
\begin{aligned}
h_{p}^{\nu}(v) & =h_{p}^{n+1}\left(e_{1}\right)=\left(\sum_{i, j=1}^{n} h_{i j}^{n+1} \omega_{i} \omega_{j}\right)\left(e_{1}\right)=\left(\sum_{i=1}^{n} \omega_{i, n+1} \omega_{i}\right)\left(e_{1}\right) \\
& =\sum_{i=1}^{n}\left\langle\bar{\nabla}_{e_{1}} e_{i}, e_{n+1}\right\rangle \omega_{i}\left(e_{1}\right)=\left\langle\bar{\nabla}_{e_{1}} e_{1}, \nu\right\rangle=-\left\langle\bar{\nabla}_{e_{1}} \nu, e_{1}\right\rangle=-\left\langle\bar{\nabla}_{v} \nu, v\right\rangle,
\end{aligned}
$$

já que $\langle v, \nu\rangle=0$. Portanto, $h^{\nu}$ não depende da escolha do referencial ortonormal e está globalmente definida.

Definição 1.8.1.3 (Imersão Geodésica) Uma imersão Riemanniana $x: M \rightarrow \bar{M}$ é geodésica em $p \in M$ se, para todo $\nu \in N_{p} M$ unitário, a segunda forma fundamental $h^{\nu}$ é identicamente nula em p. A imersão $x$ é totalmente geodésica se ela é geodésica para todo $p \in M$.

Associada à segunda forma fundamental $h_{p}^{\nu}$ em $T_{p} M$ existe uma transformação linear autoadjunta, indicada por

$$
-A_{p}^{\nu}: T_{p} M \rightarrow T_{p} M
$$


Por (1.34), temos

$$
\left\langle A_{p}^{\nu}(v), v\right\rangle=-h_{p}^{\nu}(v)=\left\langle\bar{\nabla}_{v} \nu, v\right\rangle .
$$

Associada à $-A_{p}^{\nu}$ existe uma aplicação bilinear $B_{p}: T_{p} M \times T_{p} M \rightarrow N_{p} M$ dada por

$$
\left\langle B_{p}(X, Y), \nu\right\rangle=\left\langle-A_{p}^{\nu}(X), Y\right\rangle, \quad X, Y \in T_{p} M, \quad \nu \in N_{p} M .
$$

Lema 1.8.1.4 Em termos de um referencial ortonormal local adaptado $\left\{e_{1}, \ldots, e_{n}, e_{n+1}, \ldots, e_{n+q}\right\}$, $B$ é dada por

$$
B(X, Y)=\sum_{\alpha=n+1}^{n+q}\left(\sum_{i, j=1}^{n} h_{i j}^{\alpha} \omega_{i}(X) \omega_{j}(Y)\right) e_{\alpha},
$$

o que mostra que $B$ é uma aplicação bilinear simétrica.

Demonstração: Sejam $X=\sum_{i=1}^{n} x_{i} e_{i}$ e $Y=\sum_{i=1}^{n} y_{i} e_{i}$. Temos, para cada $p \in M^{n}$,

$$
\begin{aligned}
h_{p}^{\alpha}(X+Y)= & \sum_{i, j=1}^{n} h_{i j}^{\alpha} \omega_{i}(X+Y) \omega_{j}(X+Y)=\sum_{i, j=1}^{n} h_{i j}^{\alpha} \omega_{i}(X) \omega_{j}(X)+\sum_{i, j=1}^{n} h_{i j}^{\alpha} \omega_{i}(X) \omega_{j}(Y) \\
& +\sum_{i, j=1}^{n} h_{i j}^{\alpha} \omega_{i}(Y) \omega_{j}(X)+\sum_{i, j=1}^{n} h_{i j}^{\alpha} \omega_{i}(Y) \omega_{j}(Y)=h_{p}^{\alpha}(X)+h_{p}^{\alpha}(Y)+\sum_{i, j=1}^{n} h_{i j}^{\alpha}\left(x_{i} y_{j}+y_{i} x_{j}\right) \\
= & h_{p}^{\alpha}(X)+h_{p}^{\alpha}(Y)+2 \sum_{i, j=1}^{n} h_{i j}^{\alpha} x_{i} y_{j}=h_{p}^{\alpha}(X)+h_{p}^{\alpha}(Y)+2 \sum_{i, j=1}^{n} h_{i j}^{\alpha} \omega_{i}(X) \omega_{j}(Y) .
\end{aligned}
$$

Por outro lado,

$$
\begin{aligned}
h_{p}^{\alpha}(X+Y) & =\left\langle-A_{p}^{\alpha}(X+Y), X+Y\right\rangle=\left\langle-A_{p}^{\alpha}(X), X\right\rangle+\left\langle-A_{p}^{\alpha}(X), Y\right\rangle+\left\langle-A_{p}^{\alpha}(Y), X\right\rangle+\left\langle-A_{p}^{\alpha}(Y), Y\right\rangle \\
& =h_{p}^{\alpha}(X)+h_{p}^{\alpha}(Y)+2\left\langle-A_{p}^{\alpha}(X), Y\right\rangle .
\end{aligned}
$$

Logo,

$$
\left\langle-A_{p}^{\alpha}(X), Y\right\rangle=\sum_{i, j=1}^{n} h_{i j}^{\alpha} \omega_{i}(X) \omega_{j}(Y)
$$

Portanto,

$$
B(X, Y)=\sum_{\alpha=n+1}^{n+q}\left\langle-A_{p}^{\alpha}(X), Y\right\rangle e_{\alpha}=\sum_{\alpha=n+1}^{n+q}\left(\sum_{i, j=1}^{n} h_{i j}^{\alpha} \omega_{i}(X) \omega_{j}(Y)\right) e_{\alpha},
$$

o que demonstra (1.35).

\subsubsection{Curvaturas Principal, Média e de Gauss-Kronecker}

A partir das matrizes simétricas $\left(h_{i j}^{\alpha}\right)$ definidas em (1.32) é possível obter mais três curvaturas de interesse ao estudo das variedades Riemannianas.

Definição 1.8.2.1 (Curvatura Principal) Como a matriz $\left(h_{i j}^{\alpha}\right)$ é simétrica, todos os seus autovalores são reais. Então, cada autovalor $\lambda_{i}^{\alpha}$ é chamado de curvatura principal.

Definição 1.8.2.2 (Curvatura Média) Seja $x: M^{n} \rightarrow \bar{M}^{n+q}$ uma imersão Riemanniana. Da expressão (1.35), o traço da aplicação bilinear simétrica $B$ em $p$,

$$
H_{p}=\frac{1}{n} \sum_{\alpha=n+1}^{n+q}\left(\sum_{i=1}^{n} h_{i i}^{\alpha}\right) e_{\alpha}
$$


dá origem a um vetor normal chamado vetor curvatura média em $p$.

Definição 1.8.2.3 (Imersão Mínima) Uma imersão Riemanniana $x: M \rightarrow \bar{M}$ é mínima se $H(p)=0$, para todo $p \in M$.

Definição 1.8.2.4 (Curvatura de Gauss-Kronecker) Ao valor $K^{\alpha}=\operatorname{det}\left(h_{i j}^{\alpha}\right)=\prod_{i=1}^{n} \lambda_{i}^{\alpha} d a ́$-se o nome de curvatura de Gauss-Kronecker, na direção $e_{\alpha}$.

\subsubsection{Equações de Gauss, Codazzi e Ricci}

Nesta seção serão apresentadas algumas relações importantes envolvendo o tensor curvatura e a segunda forma fundamental.

Proposição 1.8.3.1 (Equação de Gauss) Sejam $M^{n}$ e $\bar{M}^{n+q}$ variedades Riemannianas, $x: M^{n} \rightarrow \bar{M}^{n+q}$ uma imersão Riemanniana $e\left\{e_{1}, \ldots, e_{n}, e_{n+1}, \ldots, e_{n+q}\right\}$ um referencial ortonormal adaptado a $M^{n}$. Então o tensor curvatura $R_{i j k l}$ de $M^{n}$ está relacionado com as componentes tangentes $\bar{R}_{i j k l}$ do tensor curvatura de $\bar{M}^{n+q}$ por

$$
R_{i j k l}=\bar{R}_{i j k l}-\sum_{\alpha=n+1}^{n+q}\left(h_{i l}^{\alpha} h_{j k}^{\alpha}-h_{i k}^{\alpha} h_{j l}^{\alpha}\right),
$$

que é chamada de equação de Gauss.

Demonstração: Separando a parte tangencial da segunda equação de estrutura (1.28), obtém-se

$$
d \omega_{i j}=\sum_{k=1}^{n} \omega_{i k} \wedge \omega_{k j}+\sum_{\alpha=n+1}^{n+q} \omega_{i \alpha} \wedge \omega_{\alpha j}+\bar{\Omega}_{i j},
$$

ou seja,

$$
\Omega_{i j}=d \omega_{i j}-\sum_{k=1}^{n} \omega_{i k} \wedge \omega_{k j}=\sum_{\alpha=n+1}^{n+q} \omega_{i \alpha} \wedge \omega_{\alpha j}+\bar{\Omega}_{i j} .
$$

Por outro lado,

$$
\begin{aligned}
-\frac{1}{2} \sum_{k, l=1}^{n} R_{i j k l} \omega_{k} \wedge \omega_{l} & =\Omega_{i j}=\sum_{\alpha=n+1}^{n+q} \omega_{i \alpha} \wedge \omega_{\alpha j}+\bar{\Omega}_{i j}=-\sum_{\alpha=n+1}^{n+q} \omega_{i \alpha} \wedge \omega_{j \alpha}+\bar{\Omega}_{i j} \\
& =\frac{1}{2} \sum_{k, l=1}^{n}\left(\sum_{\alpha=n+1}^{n+q}\left(h_{i l}^{\alpha} h_{j k}^{\alpha}-h_{i k}^{\alpha} h_{j l}^{\alpha}\right)\right) \omega_{k} \wedge \omega_{l}-\frac{1}{2} \sum_{k, l=1}^{n} \bar{R}_{i j k l} \omega_{k} \wedge \omega_{l}
\end{aligned}
$$

ou seja,

$$
R_{i j k l}=\bar{R}_{i j k l}-\sum_{\alpha=n+1}^{n+q}\left(h_{i l}^{\alpha} h_{j k}^{\alpha}-h_{i k}^{\alpha} h_{j l}^{\alpha}\right),
$$

o que demonstra (1.36).

Corolário 1.8.3.2 Sejam $M^{n}$ uma variedade Riemanniana, $\bar{M}^{n+1}$ uma variedade Riemanniana com curvatura seccional constante $c, x: M^{n} \rightarrow \bar{M}^{n+1}$ uma imersão Riemanniana e $\left\{e_{1}, \ldots, e_{n+1}\right\}$ um referencial ortonormal adaptado a $M^{n}$. Então a equação de Gauss se escreve como

$$
R_{i j k l}=c\left(\delta_{i k} \delta_{j l}-\delta_{i l} \delta_{j k}\right)+h_{i k} h_{j l}-h_{i l} h_{j k} .
$$

Demonstração: Como $\bar{M}^{n+1}$ tem curvatura seccional constante $c$, por (1.21) e (1.36), segue o resultado. 
Corolário 1.8.3.3 Sejam $M^{n}$ uma variedade Riemanniana, $\bar{M}^{n+1}$ uma variedade Riemanniana com curvatura seccional constante $c, x: M^{n} \rightarrow \bar{M}^{n+1}$ uma imersão Riemanniana mínima $e$ $\left\{e_{1}, \ldots, e_{n+1}\right\}$ um referencial ortonormal adaptado a $M^{n}$. Então a curvatura de Ricci e curvatura escalar de $M^{n}$, valem, respectivamente,

$$
\begin{gathered}
R_{i j}=(n-1) c \delta_{i j}-\sum_{k=1}^{n} h_{i k} h_{k j}, \\
R=n(n-1) c-S,
\end{gathered}
$$

onde $S=\sum_{i, j=1}^{n} h_{i j}^{2}$ é o quadrado da norma de $h$. De (1.39) segue que a curvatura escalar é constante se, e somente se, $S$ é constante.

Demonstração: Por (1.22), (1.37) e pelo fato de $M^{n}$ ser mínima, temos

$$
\begin{aligned}
R_{i j} & =\sum_{k=1}^{n} R_{i k j k}=\sum_{k=1}^{n}\left(c\left(\delta_{i j} \delta_{k k}-\delta_{i k} \delta_{k j}\right)+h_{i j} h_{k k}-h_{i k} h_{k j}\right) \\
& =n c \delta_{i j}-c \delta_{i j}+H h_{i j}-\sum_{k=1}^{n} h_{i k} h_{k j}=(n-1) c \delta_{i j}-\sum_{k=1}^{n} h_{i k} h_{k j},
\end{aligned}
$$

o que demonstra (1.38). Agora, de (1.23) e (1.38), temos

$$
R=\sum_{i=1}^{n} R_{i i}=\sum_{i=1}^{n}\left((n-1) c \delta_{i i}-\sum_{k=1}^{n} h_{i k} h_{k i}\right)=n(n-1) c-\sum_{i, k=1}^{n} h_{i k}^{2}=n(n-1) c-S,
$$

o que demonstra (1.39).

Proposição 1.8.3.4 (Equação de Codazzi) Sejam $M^{n}$ uma variedade Riemanniana, $\bar{M}^{n+1}$ uma variedade Riemanniana com curvatura seccional constante $c, x: M^{n} \rightarrow \bar{M}^{n+1}$ uma imersão Riemanniana e $\left\{e_{1}, \ldots, e_{n+1}\right\}$ um referencial ortonormal adaptado a $M^{n}$. A diferencial covariante $\nabla h$ da segunda forma fundamental $h$ de $M^{n}$ com componentes $h_{i j ; k}$ é dada por

$$
\sum_{k=1}^{n} h_{i j ; k} \omega_{k}=d h_{i j}+\sum_{k=1}^{n} h_{k j} \omega_{k i}+\sum_{k=1}^{n} h_{i k} \omega_{k j} .
$$

Então vale a seguinte relação, chamada equação de Codazzi,

$$
h_{i j ; k}=h_{i k ; j}=h_{j i ; k} .
$$

Demonstração: Começamos derivando exteriormente a expressão (1.33), utilizando (1.30)

$$
d \omega_{i, n+1}=\sum_{j=1}^{n} d h_{i j} \wedge \omega_{j}+\sum_{j=1}^{n} h_{i j} d \omega_{j}=\sum_{j=1}^{n} d h_{i j} \wedge \omega_{j}+\sum_{j, k=1}^{n} h_{i j} \omega_{j k} \wedge \omega_{k} .
$$

Trocando o papel de $j$ e $k$ na última parcela da equação acima, obtemos

$$
d \omega_{i, n+1}=\sum_{j=1}^{n} d h_{i j} \wedge \omega_{j}+\sum_{j, k=1}^{n} h_{i k} \omega_{k j} \wedge \omega_{j}
$$

Por outro lado, de (1.29) e utilizando a antissimetria das formas $\omega_{A B}$, temos 


$$
d \omega_{i, n+1}=\sum_{A=1}^{n+1} \omega_{i A} \wedge \omega_{A, n+1}+\bar{\Omega}_{i, n+1}=\sum_{k=1}^{n} \omega_{i k} \wedge \omega_{k, n+1}+\omega_{i, n+1} \wedge \omega_{n+1, n+1}+\bar{\Omega}_{i, n+1},
$$

ou seja,

$$
d \omega_{i, n+1}=\sum_{k=1}^{n} \omega_{i k} \wedge \omega_{k, n+1}+\bar{\Omega}_{i, n+1} .
$$

Restrito a vetores tangentes a $M^{n}$, utilizando (1.21) e (1.29), temos

$$
\begin{aligned}
\bar{\Omega}_{i, n+1}= & -\frac{1}{2} \sum_{C, D=1}^{n+1} \bar{R}_{i, n+1, C, D} \omega_{C} \wedge \omega_{D}=-\frac{1}{2} \sum_{k, l=1}^{n} \bar{R}_{i, n+1, k, l} \omega_{k} \wedge \omega_{l} \\
& -\frac{1}{2} \sum_{k=1} \bar{R}_{i, n+1, k, n+1} \omega_{k} \wedge \omega_{n+1}-\frac{1}{2} \bar{R}_{i, n+1, n+1, n+1} \omega_{n+1} \wedge \omega_{n+1} \\
= & -\frac{1}{2} \sum_{k, l=1}^{n} \bar{R}_{i, n+1, k, l} \omega_{k} \wedge \omega_{l}=-\frac{1}{2} \sum_{k, l=1}^{n} c\left(\delta_{i k} \delta_{n+1, l}-\delta_{i l} \delta_{n+1, k}\right) \omega_{k} \wedge \omega_{l}=0 .
\end{aligned}
$$

Logo, quando restrito a vetores tangentes a $M^{n}$, por (1.43) e (1.44) obtemos

$$
d \omega_{i, n+1}=\sum_{k=1}^{n} \omega_{i k} \wedge \omega_{k, n+1} .
$$

Multiplicando exteriormente (1.40) por $\omega_{j}$, somando em $j$ e utilizando (1.33), (1.42) e (1.45), temos

$$
\begin{aligned}
\sum_{j, k=1}^{n} h_{i j ; k} \omega_{k} \wedge \omega_{j} & =\sum_{j=1}^{n} d h_{i j} \wedge \omega_{j}+\sum_{j, k=1}^{n} h_{k j} \omega_{k i} \wedge \omega_{j}+\sum_{j, k=1}^{n} h_{i k} \omega_{k j} \wedge \omega_{j} \\
& =\sum_{j=1}^{n} d h_{i j} \wedge \omega_{j}+\sum_{k=1}^{n} \omega_{k i} \wedge\left(\sum_{j=1}^{n} h_{k j} \omega_{j}\right)+\sum_{j, k=1}^{n} h_{i k} \omega_{k j} \wedge \omega_{j} \\
& =\sum_{j=1}^{n} d h_{i j} \wedge \omega_{j}+\sum_{k=1}^{n} \omega_{k i} \wedge \omega_{k, n+1}+\sum_{j, k=1}^{n} h_{i k} \omega_{k j} \wedge \omega_{j} \\
& =\sum_{j=1}^{n} d h_{i j} \wedge \omega_{j}-\sum_{k=1}^{n} \omega_{i k} \wedge \omega_{k, n+1}+\sum_{j, k=1}^{n} h_{i k} \omega_{k j} \wedge \omega_{j} \\
& =\sum_{j=1}^{n} d h_{i j} \wedge \omega_{j}-d \omega_{i, n+1}+\sum_{j, k=1}^{n} h_{i k} \omega_{k j} \wedge \omega_{j} \\
& =\sum_{j=1}^{n} d h_{i j} \wedge \omega_{j}-\left(\sum_{j=1}^{n} d h_{i j} \wedge \omega_{j}+\sum_{j, k=1}^{n} h_{i k} \omega_{k j} \wedge \omega_{j}\right)+\sum_{j, k=1}^{n} h_{i k} \omega_{k j} \wedge \omega_{j} \\
& =0 .
\end{aligned}
$$

Logo, como $\omega_{k} \wedge \omega_{j}, k<j$, são linearmente independentes,

$$
0=\sum_{j, k=1}^{n} h_{i j ; k} \omega_{k} \wedge \omega_{j}=\sum_{\substack{j, k=1 \\ k<j}}^{n}\left(h_{i j ; k}-h_{i k ; j}\right) \omega_{k} \wedge \omega_{j}
$$

ou seja,

$$
h_{i j ; k}=h_{i k ; j} .
$$


Como $h_{i j}=h_{j i}$, de (1.40) vale

$$
\begin{aligned}
\sum_{k=1}^{n} h_{i j ; k} \omega_{k} & =d h_{i j}+\sum_{k=1}^{n} h_{k j} \omega_{k i}+\sum_{k=1}^{n} h_{i k} \omega_{k j} \\
& =d h_{j i}+\sum_{k=1}^{n} h_{j k} \omega_{k i}+\sum_{k=1}^{n} h_{k i} \omega_{k j} \\
& =d h_{j i}+\sum_{k=1}^{n} h_{k i} \omega_{k j}+\sum_{k=1}^{n} h_{j k} \omega_{k i}=\sum_{k=1}^{n} h_{j i ; k} \omega_{k} .
\end{aligned}
$$

Logo,

$$
h_{i j ; k}=h_{j i ; k}
$$

o que demonstra (1.41).

Proposição 1.8.3.5 (Equação de Ricci) Sejam $M^{n}$ e $\bar{M}^{n+1}$ variedades Riemannianas, $x: M^{n} \rightarrow \bar{M}^{n+1}$ uma imersão Riemanniana $e\left\{e_{1}, \ldots, e_{n+1}\right\}$ um referencial ortonormal adaptado a $M^{n}$. A segunda diferencial covariante $\nabla^{2} h$ da segunda forma fundamental $h$ de $M^{n}$ com componentes $h_{i j k ; l}$ é dada por

$$
\sum_{l=1}^{n} h_{i j k ; l} \omega_{l}=d h_{i j ; k}+\sum_{l=1}^{n} h_{l j ; k} \omega_{l i}+\sum_{l=1}^{n} h_{i l ; k} \omega_{l j}+\sum_{l=1}^{n} h_{i j ; l} \omega_{l k} .
$$

Então vale a seguinte relação, chamada equação de Ricci,

$$
h_{i j k ; l}-h_{i j l ; k}=\sum_{m=1}^{n} h_{i m} R_{m j k l}+\sum_{m=1}^{n} h_{m j} R_{m i k l} .
$$

Demonstração: Por (1.40), temos

$$
d h_{i j}=\sum_{k=1}^{n} h_{i j ; k} \omega_{k}-\sum_{k=1}^{n} h_{k j} \omega_{k i}-\sum_{k=1}^{n} h_{i k} \omega_{k j} .
$$

Derivando exteriormente (1.40) e observando que $d h_{i j}$ é uma 1-forma exata, obtemos

$$
\begin{aligned}
\sum_{k=1}^{n} d h_{i j ; k} \wedge \omega_{k}+\sum_{k=1}^{n} h_{i j ; k} d \omega_{k} & =d^{2} h_{i j}+\sum_{k=1}^{n} d h_{k j} \wedge \omega_{k i}+\sum_{k=1}^{n} h_{k j} d \omega_{k i}+\sum_{k=1}^{n} d h_{i k} \wedge \omega_{k j}+\sum_{k=1}^{n} h_{i k} d \omega_{k j} \\
& =\sum_{k=1}^{n} d h_{k j} \wedge \omega_{k i}+\sum_{k=1}^{n} h_{k j} d \omega_{k i}+\sum_{k=1}^{n} d h_{i k} \wedge \omega_{k j}+\sum_{k=1}^{n} h_{i k} d \omega_{k j}
\end{aligned}
$$

ou seja, isolando a primeira parcela e utilizando $l$ nos somatórios à direita da igualdade,

$$
\begin{aligned}
\sum_{k=1}^{n} d h_{i j ; k} \wedge \omega_{k}= & \sum_{l=1}^{n} d h_{l j} \wedge \omega_{l i}+\sum_{l=1}^{n} h_{l j} d \omega_{l i} \\
& +\sum_{l=1}^{n} d h_{i l} \wedge \omega_{l j}+\sum_{l=1}^{n} h_{i l} d \omega_{l j}-\sum_{l=1}^{n} h_{i j ; l} d \omega_{l} .
\end{aligned}
$$

Utilizando (1.30), (1.31) e (1.48) na expressão acima, obtemos 


$$
\begin{aligned}
\sum_{k=1}^{n} d h_{i j ; k} \wedge \omega_{k}= & \sum_{l=1}^{n}\left(\sum_{k=1}^{n} h_{l j ; k} \omega_{k}-\sum_{k=1}^{n} h_{k j} \omega_{k l}-\sum_{k=1}^{n} h_{l k} \omega_{k j}\right) \wedge \omega_{l i} \\
& +\sum_{l=1}^{n} h_{l j}\left(\sum_{k=1}^{n} \omega_{l k} \wedge \omega_{k i}-\frac{1}{2} \sum_{k, m=1}^{n} R_{l i k m} \omega_{k} \wedge \omega_{m}\right) \\
& +\sum_{l=1}^{n}\left(\sum_{k=1}^{n} h_{i l ; k} \omega_{k}-\sum_{k=1}^{n} h_{k l} \omega_{k i}-\sum_{k=1}^{n} h_{i k} \omega_{k l}\right) \wedge \omega_{l j} \\
& +\sum_{l=1}^{n} h_{i l}\left(\sum_{k=1}^{n} \omega_{l k} \wedge \omega_{k j}-\frac{1}{2} \sum_{k, m=1}^{n} R_{l j k m} \omega_{k} \wedge \omega_{m}\right)-\sum_{l=1}^{n} h_{i j ; l}\left(\sum_{k=1}^{n} \omega_{l k} \wedge \omega_{k}\right) \\
= & \sum_{k, l=1}^{n} h_{l j ; k} \omega_{k} \wedge \omega_{l i}-\sum_{k, l=1}^{n} h_{k j} \omega_{k l} \wedge \omega_{l i}-\sum_{k, l=1}^{n} h_{l k} \omega_{k j} \wedge \omega_{l i} \\
& +\sum_{k, l=1}^{n} h_{l j} \omega_{l k} \wedge \omega_{k i}-\frac{1}{2} \sum_{k, l, m=1}^{n} h_{l j} R_{l i k m} \omega_{k} \wedge \omega_{m} \\
& +\sum_{k, l=1}^{n} h_{i l ; k} \omega_{k} \wedge \omega_{l j}-\sum_{k, l=1}^{n} h_{k l} \omega_{k i} \wedge \omega_{l j}-\sum_{k, l=1}^{n} h_{i k} \omega_{k l} \wedge \omega_{l j} \\
& +\sum_{k, l=1}^{n} h_{i l} \omega_{l k} \wedge \omega_{k j}-\frac{1}{2} \sum_{k, l, m=1}^{n} h_{i l} R_{l j k m} \omega_{k} \wedge \omega_{m}-\sum_{k, l=1}^{n} h_{i j ; l} \omega_{l k} \wedge \omega_{k} \\
= & \sum_{k, l=1}^{n} h_{l j ; k} \omega_{k} \wedge \omega_{l i}-\frac{1}{2} \sum_{k, l, m=1}^{n} h_{l j} R_{l i k m} \omega_{k} \wedge \omega_{m} \\
& +\sum_{k, l=1}^{n} h_{i l ; k} \omega_{k} \wedge \omega_{l j}-\frac{1}{2} \sum_{k, l, m=1}^{n} h_{i l} R_{l j k m} \omega_{k} \wedge \omega_{m}-\sum_{k, l=1}^{n} h_{i j ; l} \omega_{l k} \wedge \omega_{k} .
\end{aligned}
$$

Portanto, trocando $m$ com $l$ nos somatórios envolvendo o operador de curvatura,

$$
\begin{aligned}
\sum_{k=1}^{n} d h_{i j ; k} \wedge \omega_{k}= & \frac{1}{2} \sum_{k, l, m=1}^{n} h_{m j} R_{m i k l} \omega_{l} \wedge \omega_{k}+\frac{1}{2} \sum_{k, l, m=1}^{n} h_{i m} R_{m j k l} \omega_{l} \wedge \omega_{k} \\
& -\sum_{k, l=1}^{n} h_{l j ; k} \omega_{l i} \wedge \omega_{k}-\sum_{k, l=1}^{n} h_{i l ; k} \omega_{l j} \wedge \omega_{k}-\sum_{k, l=1}^{n} h_{i j ; l} \omega_{l k} \wedge \omega_{k} .
\end{aligned}
$$

Multiplicando exteriormente (1.46) por $\omega_{k}$, somando em $k$ e utilizando a expressão (1.49), obtemos

$$
\begin{aligned}
\sum_{k, l=1}^{n} h_{i j k ; l} \omega_{l} \wedge \omega_{k}= & \sum_{k=1}^{n} d h_{i j ; k} \wedge \omega_{k}+\sum_{k, l=1}^{n} h_{l j ; k} \omega_{l i} \wedge \omega_{k}+\sum_{k, l=1}^{n} h_{i l ; k} \omega_{l j} \wedge \omega_{k}+\sum_{k, l=1}^{n} h_{i j ; l l} \omega_{l k} \wedge \omega_{k} \\
= & \frac{1}{2} \sum_{k, l, m=1}^{n} h_{m j} R_{m i k l} \omega_{l} \wedge \omega_{k}+\frac{1}{2} \sum_{k, l, m=1}^{n} h_{i m} R_{m j k l} \omega_{l} \wedge \omega_{k} \\
& -\sum_{k, l=1}^{n} h_{l j ; k} \omega_{l i} \wedge \omega_{k}-\sum_{k, l=1}^{n} h_{i l ; k} \omega_{l j} \wedge \omega_{k}-\sum_{k, l=1}^{n} h_{i j ; l} \omega_{l k} \wedge \omega_{k} \\
& +\sum_{k, l=1}^{n} h_{l j ; k} \omega_{l i} \wedge \omega_{k}+\sum_{k, l=1}^{n} h_{i l ; k} \omega_{l j} \wedge \omega_{k}+\sum_{k, l=1}^{n} h_{i j ; l} \omega_{l k} \wedge \omega_{k}
\end{aligned}
$$




$$
=\frac{1}{2} \sum_{k, l, m=1}^{n} h_{m j} R_{m i k l} \omega_{l} \wedge \omega_{k}+\frac{1}{2} \sum_{k, l, m=1}^{n} h_{i m} R_{m j k l} \omega_{l} \wedge \omega_{k} .
$$

Logo,

$$
\sum_{\substack{k, l=1 \\ l<k}}^{n}\left(h_{i j k ; l}-h_{i j l ; k}\right) \omega_{l} \wedge \omega_{k}=\sum_{\substack{k, l, m=1 \\ l<k}}^{n} h_{m j} R_{m i k l} \omega_{l} \wedge \omega_{k}+\sum_{\substack{k, l, m=1 \\ l<k}}^{n} h_{i m} R_{m j k l} \omega_{l} \wedge \omega_{k}
$$

Como $\omega_{l} \wedge \omega_{k}, l<k$, são linearmente independentes, temos

$$
h_{i j k ; l}-h_{i j l ; k}=\sum_{m=1}^{n} h_{m j} R_{m i k l}+\sum_{m=1}^{n} h_{i m} R_{m j k l},
$$

o que demonstra (1.47).

Corolário 1.8.3.6 Sejam $M^{n}$ uma variedade Riemanniana, $\bar{M}^{n+1}$ uma variedade Riemanniana com curvatura seccional constante $c, x: M^{n} \rightarrow \bar{M}^{n+1}$ uma imersão Riemanniana e $\left\{e_{1}, \ldots, e_{n+1}\right\}$ um referencial ortonormal adaptado a $M^{n}$. Sejam, respectivamente, $h_{i j ; k}$ e $h_{i j k ; l}$ as componentes da primeira e da segunda diferencial covariante da segunda forma fundamental h. Então vale

$$
h_{i j ; k}=h_{i k ; j}=h_{j i ; k} \Longrightarrow h_{i j k ; l}=h_{i k j ; l}=h_{j i k ; l}, \quad \forall l=1, \ldots n .
$$

Demonstração: O lado esquerdo da implicação trata-se de (1.41). Mostraremos que se $h_{i j ; k}=h_{i k ; j}$, então $h_{i j k ; l}=h_{i k j ; l}, \forall l=1, \ldots n$, sendo que os outros casos são análogos. De fato, por $(1.46)$,

$$
\begin{aligned}
\sum_{l=1}^{n} h_{i j k ; l} \omega_{l} & =d h_{i j ; k}+\sum_{l=1}^{n} h_{l j ; k} \omega_{l i}+\sum_{l=1}^{n} h_{i l ; k} \omega_{l j}+\sum_{l=1}^{n} h_{i j ; l} \omega_{l k} \\
& =d h_{i k ; j}+\sum_{l=1}^{n} h_{l k ; j} \omega_{l i}+\sum_{l=1}^{n} h_{i k ; l} \omega_{l j}+\sum_{l=1}^{n} h_{i l ; j} \omega_{l k} \\
& =d h_{i k ; j}+\sum_{l=1}^{n} h_{l k ; j} \omega_{l i}+\sum_{l=1}^{n} h_{i l ; j} \omega_{l k}+\sum_{l=1}^{n} h_{i k ; l} \omega_{l j}=\sum_{l=1}^{n} h_{i k j ; l} \omega_{l}
\end{aligned}
$$

o que demonstra o afirmado.

Corolário 1.8.3.7 Sejam $M^{n}$ uma variedade Riemanniana, $\bar{M}^{n+1}$ uma variedade Riemanniana com curvatura seccional constante c e $x: M^{n} \rightarrow \bar{M}^{n+1}$ uma imersão Riemanniana mínima. Fixado qualquer ponto $p \in M^{n}$, como $\left(h_{i j}\right)$ é simétrica, é possivel escolher um referencial ortonormal $\left\{e_{1}, \ldots, e_{n+1}\right\}$ adaptado a $M^{n}$, de modo que $\left(h_{i j}\right)$ esteja na forma diagonal e valha

$$
h_{i j}=\lambda_{i} \delta_{i j} .
$$

Nessas condições, podemos escrever

$$
h_{i j i ; j}-h_{j i j ; i}=\left(\lambda_{i}-\lambda_{j}\right)\left(c+\lambda_{i} \lambda_{j}\right) .
$$

Demonstração: Do Corolário 1.8.3.6, $h_{i j j ; i}=h_{j i j ; i}$. Logo, por (1.37), (1.47) e (1.51), temos

$$
h_{i j i ; j}-h_{j i j ; i}=h_{i j i ; j}-h_{i j j ; i}=\sum_{m=1}^{n} h_{i m} R_{m j i j}+\sum_{m=1}^{n} h_{m j} R_{m i i j}
$$




$$
\begin{aligned}
= & \sum_{m=1}^{n} h_{i m}\left(c\left(\delta_{m i} \delta_{j j}-\delta_{m j} \delta_{j i}\right)+h_{m i} h_{j j}-h_{m j} h_{j i}\right) \\
& +\sum_{m=1}^{n} h_{m j}\left(c\left(\delta_{m i} \delta_{i j}-\delta_{m j} \delta_{i i}\right)+h_{m i} h_{i j}-h_{m j} h_{i i}\right) \\
= & c h_{i i}-c h_{j i} \delta_{i j}+\sum_{m=1}^{n} h_{i m} h_{m i} h_{j j}-\sum_{m=1}^{n} h_{i m} h_{m j} h_{j i} \\
& +c h_{i j} \delta_{i j}-c h_{j j}+\sum_{m=1}^{n} h_{m j} h_{m i} h_{i j}-\sum_{m=1}^{n} h_{m j} h_{m j} h_{i i} \\
= & c h_{i i}+\sum_{m=1}^{n} h_{i m}^{2} h_{j j}-c h_{j j}-\sum_{m=1}^{n} h_{j m}^{2} h_{i i} \\
= & c \lambda_{i} \delta_{i i}+\sum_{m=1}^{n}\left(\lambda_{i} \delta_{i m}\right)^{2} \lambda_{j} \delta_{j j}-c \lambda_{j} \delta_{j j}-\sum_{m=1}^{n}\left(\lambda_{j} \delta_{j m}\right)^{2} \lambda_{i} \delta_{i i} \\
= & c \lambda_{i}+\lambda_{i}^{2} \lambda_{j}-c \lambda_{j}-\lambda_{j}^{2} \lambda_{i}=\lambda_{i}\left(c+\lambda_{i} \lambda_{j}\right)-\lambda_{j}\left(c+\lambda_{i} \lambda_{j}\right) \\
= & \left(\lambda_{i}-\lambda_{j}\right)\left(c+\lambda_{i} \lambda_{j}\right),
\end{aligned}
$$

o que demonstra (1.52).

Proposição 1.8.3.8 (Simons [26]) Sejam $M^{n}$ uma variedade Riemanniana, $\bar{M}^{n+1}$ uma variedade Riemanniana com curvatura seccional constante c, $x: M^{n} \rightarrow \bar{M}^{n+1}$ uma imersão Riemanniana minima e $\left\{e_{1}, \ldots, e_{n+1}\right\}$ um referencial ortonormal adaptado a $M^{n}$. Então o Laplaciano $\Delta h_{i j}$, das componentes $h_{i j}$ da segunda forma fundamental $h$, é dado por

$$
\Delta h_{i j}=\sum_{k=1}^{n} h_{i j k ; k}=(n c-S) h_{i j}
$$

Além disso,

$$
\frac{1}{2} \Delta S=(n c-S) S+\sum_{i, j, k=1}^{n} h_{i j ; k}^{2},
$$

onde $S=\sum_{i, j=1}^{n} h_{i j}^{2}$ é o quadrado da norma de $h$.

Demonstração: Como a imersão é mínima, temos

$$
\operatorname{grad} H=\sum_{i=1}^{n} H_{i} e_{i}=0 \quad e \quad \operatorname{Hess} H=\sum_{i, j=1}^{n} H_{i ; j} \omega_{j}=0,
$$

e concluímos que

$$
H_{i ; j}=\sum_{k=1}^{n} h_{k k i ; j}=0, \quad \forall i, j=1, \ldots, n .
$$

Por (1.37), (1.47), (1.50), (1.55) e pelo fato de $M$ ser mínima, segue que

$$
\begin{aligned}
\Delta h_{i j} & =\sum_{k=1}^{n} h_{i j k ; k}=\sum_{k=1}^{n} h_{i k j ; k} \\
& =\sum_{k=1}^{n}\left(h_{i k j ; k}+\left(h_{i k k ; j}-h_{i k k ; j}\right)\right)
\end{aligned}
$$




$$
\begin{aligned}
= & \sum_{k=1}^{n}\left(h_{i k j ; k}-h_{i k k ; j}\right)+\sum_{k=1}^{n} h_{i k k ; j} \\
= & \sum_{k=1}^{n}\left(h_{i k j ; k}-h_{i k k ; j}\right)+\sum_{k=1}^{n} h_{k k i ; j} \\
= & \sum_{k=1}^{n}\left(h_{i k j ; k}-h_{i k k ; j}\right) \\
= & \sum_{k=1}^{n}\left(\sum_{m=1}^{n} h_{i m} R_{m k j k}+\sum_{m=1}^{n} h_{m k} R_{m i j k}\right) \\
= & \sum_{k=1}^{n}\left(\sum_{m=1}^{n} h_{i m}\left(c\left(\delta_{m j} \delta_{k k}-\delta_{m k} \delta_{k j}\right)+h_{m j} h_{k k}-h_{m k} h_{k j}\right)\right. \\
& \left.+\sum_{m=1}^{n} h_{m k}\left(c\left(\delta_{m j} \delta_{i k}-\delta_{m k} \delta_{i j}\right)+h_{m j} h_{i k}-h_{m k} h_{i j}\right)\right) \\
= & n c h_{i j}-c h_{i j}+\sum_{k, m=1}^{n} h_{i m} h_{m j} h_{k k}-\sum_{k, m=1}^{n} h_{i m} h_{m k} h_{k j} \\
& +c h_{j i}-\sum_{k=1}^{n} c h_{k k} \delta_{i j}+\sum_{k, m=1}^{n} h_{m k} h_{m j} h_{i k}-\sum_{k, m=1}^{n} h_{m k}^{2} h_{i j} \\
= & n c h_{i j}+H \sum_{m=1}^{n} h_{i m} h_{m j}-H c \delta_{i j}-S h_{i j} \\
= & (n c-S) h_{i j}, \quad
\end{aligned}
$$

o que demonstra (1.53). Para provar (1.54), utilizando (1.27) e (1.53), segue que

$$
\begin{aligned}
\Delta S & =\Delta\left(\sum_{i, j=1}^{n} h_{i j}^{2}\right)=\sum_{i, j=1}^{n} \Delta\left(h_{i j}^{2}\right)=2 \sum_{i, j=1}^{n}\left(h_{i j} \Delta h_{i j}+\left\langle\operatorname{grad} h_{i j}, \operatorname{grad} h_{i j}\right\rangle\right) \\
& =2 \sum_{i, j=1}^{n} h_{i j} \Delta h_{i j}+2 \sum_{i, j, k=1}^{n} h_{i j ; k}^{2}=2(n c-S) S+2 \sum_{i, j, k=1}^{n} h_{i j ; k}^{2} .
\end{aligned}
$$

\subsection{Os Espaços Forma $\mathbb{Q}^{n}(c)$}

As variedades Riemannianas que apresentam curvatura seccional constante são clássicas e tem uma geometria mais simples.

Definição 1.9.1 (Espaço Forma) Um espaço forma é uma variedade Riemanniana completa com curvatura seccional constante.

Ao longo deste texto, $\mathbb{Q}^{n}(c)$ denotará um espaço forma n-dimensional simplesmente conexo, com curvatura seccional constante $c$. Se $c>0, \mathbb{Q}^{n}(c)$ é a esfera $\mathbb{S}^{n}(c)$, se $c=0$, é o espaço Euclidiano $n$-dimensional $\mathbb{R}^{n}$, e se $c<0$, é o espaço hiperbólico $n$-dimensional $\mathbb{H}^{n}(c)$. A menos de uma semelhança, é possível supor que a curvatura seccional constante vale $1,0 \mathrm{ou}-1$, e, nestes casos, as notações são, respectivamente, $\mathbb{S}^{n}, \mathbb{R}^{n}$ ou $\mathbb{H}^{n}$.

Assim como feito por Asperti et al. em [1], a seguir apresentaremos brevemente os espaços hiperbólico $\mathbb{H}^{n}$ e de De Sitter $\mathbb{S}_{1}^{n}$. 
Definição 1.9.2 (Espaço Lorentziano) $O$ espaço Lorentziano, denotado por $\mathbb{R}_{1}^{n+1}$, é o espaço vetorial real $\mathbb{R}^{n+1}$, munido com o tensor métrico Lorentziano $\langle$,$\rangle dado por$

$$
\langle x, y\rangle=-x_{0} y_{0}+\sum_{i=1}^{n} x_{i} y_{i}
$$

onde $x=\left(x_{0}, x_{1}, \ldots, x_{n}\right), y=\left(y_{0}, y_{1}, \ldots, y_{n}\right) \in \mathbb{R}^{n+1}$.

Observação 1.9.3 No espaço Lorentziano é possível classificar um campo de vetores $w$ em uma das seguintes categorias:

(i) tipo-espaço, se $\langle w, w\rangle>0$ ou $w=0$;

(ii) tipo-tempo, se $\langle w, w\rangle<0$;

(iii) tipo-luz, se $\langle w, w\rangle=0$ e $w \neq 0$.

\subsubsection{O Espaço Hiperbólico $\mathbb{H}^{n}$}

O modelo de Minkowski para o espaço hiperbólico simplesmente conexo de curvatura seccional constante -1 é a hiperquádrica

$$
\mathbb{H}^{n}=\left\{x \in \mathbb{R}_{1}^{n+1} ;\langle x, x\rangle=-1, x_{0}>0\right\} .
$$

O resultado a seguir, presente no Capítulo 2 de [28], apresenta como obter as subvariedades umbílicas do espaço hiperbólico.

Proposição 1.9.1.1 (Suzuki [28]) As esferas geodésicas, as horoesferas, as superfícies equidistantes e as superfícies totalmente geodésicas são as subvariedades umbílicas de $\mathbb{H}^{n}$ e são obtidas tomando-se intersecções de $\mathbb{H}^{n}$ com subespaços afins de $\mathbb{R}_{1}^{n+1}$.

\subsubsection{O Espaço de De Sitter $\mathbb{S}_{1}^{n}$}

A hiperquádrica de $\mathbb{R}_{1}^{n+1}$ dada por

$$
\mathbb{S}_{1}^{n}=\left\{x \in \mathbb{R}_{1}^{n+1} ;\langle x, x\rangle=1\right\}
$$

é um modelo padrão para o espaço forma Lorentziano com curvatura constante 1, e é chamado de espaço de De Sitter.

Alguns dos resultados discutidos por Asperti et al. em [1] tratam especificamente do caso de imersões de variedades Riemannianas bidimensionais em $\mathbb{S}_{1}^{4}$, e por isso introduziremos as seguintes definições.

Definição 1.9.2.1 (Imersão Tipo-Espaço) Uma imersão $g: M^{2} \rightarrow \mathbb{S}_{1}^{4}$ é chamada de tipo-espaço se a métrica induzida em $M^{2}$, por $g$, for Riemanniana. (É usual continuar a denotar a métrica induzida também por $\langle\rangle$,$) .$

Definição 1.9.2.2 (Fibrado Normal) Sejam $g: M^{2} \rightarrow \mathbb{S}_{1}^{4}$ uma imersão de tipo-espaço e $i: \mathbb{S}_{1}^{4} \rightarrow \mathbb{R}_{1}^{5}$ a aplicação inclusão. Denote por

$$
\begin{aligned}
(i \circ g)^{*}\left(T \mathbb{R}_{1}^{5}\right) & =\left\{(p, w) ; p \in M^{2}, w \in T_{i \circ g(p)} \mathbb{R}_{1}^{5}\right\}, \\
g^{*}\left(T \mathbb{S}_{1}^{4}\right) & =\left\{(p, w) ; p \in M^{2}, w \in T_{g(p)} \mathbb{S}_{1}^{4}\right\},
\end{aligned}
$$

os fibrados induzidos de, respectivamente, $i \circ g$ e g. Desta forma, o fibrado normal $\mathcal{N}(g)$ de $g$ é dado por

$$
\mathcal{N}(g)=\left\{(p, w) \in g^{*}\left(T \mathbb{S}_{1}^{4}\right) ; w \perp d g\left(T_{p} M^{2}\right)\right\} .
$$


Definição 1.9.2.3 (Imersão Estacionária) Seja $g: M^{2} \rightarrow \mathbb{S}_{1}^{4}$ uma imersão de tipo-espaço isométrica. Tal imersão é chamada de estacionária se o vetor curvatura média $H$ de $g$ for identicamente zero.

Definição 1.9.2.4 (Aplicação Normal) Sejam $g: M^{2} \rightarrow \mathbb{S}_{1}^{4}$ uma imersão de tipo-espaço estacionária isométrica e $M^{2}$ uma variedade orientada. Considere o fibrado normal unitário tipo-tempo $\mathcal{N}^{1}(g)$ de $g$, definido por

$$
\mathcal{N}^{1}(g)=\{(p, w) \in \mathcal{N}(g) ;\langle w, w\rangle=-1\} .
$$

Denote por $\pi: \mathcal{N}^{1}(g) \rightarrow M^{2}$ a projeção no primeiro fator e por $\psi_{g}: \mathcal{N}^{1}(g) \rightarrow \mathbb{H}^{4}$ a projeção no segundo fator. A aplicação $\psi_{g}$ é chamada de aplicação normal associada a $g$. 


\section{Capítulo 2}

\section{Os Casos Euclideano $\mathbb{R}^{4}$ e Hiperbólico $\mathbb{H}^{4}$}

Neste capítulo apresentamos os resultados obtidos por Asperti et al. em [1] referentes a imersões de hipersuperfícies tridimensionais em espaços forma quadridimensionais $\mathbb{Q}^{4}(c)$, com $c \leq 0$.

Começamos com uma definição dada por Takegoshi em [29], acerca de funções suaves definidas em uma variedade Riemanniana.

Definição 2.1 Uma função suave $f$, definida em uma variedade Riemanniana $M$, é dita fortemente super-harmônica (respectivamente super-harmônica) se $f$ satisfaz em $M$ a seguinte inequação diferencial:

$$
\Delta f=\operatorname{div} \operatorname{grad} f<0 \quad \text { (respectivamente } \Delta f \leq 0) .
$$

Na prova do Teorema 2.7 é utilizada uma generalização do Princípio do Máximo de Yau [32], que será apresentada no lema a seguir.

Lema 2.2 Seja $M^{n}$ uma variedade Riemanniana completa $n$-dimensional com curvatura de Ricci limitada inferiormente e seja $f: M^{n} \rightarrow \mathbb{R}$ uma função de classe $C^{2}$, que é limitada inferiormente em $M^{n}$. Então existe uma sequência de pontos $\left\{p_{k}\right\}_{k \in \mathbb{N}}$ em $M^{n}$ tal que

$$
\lim _{k \rightarrow \infty} f\left(p_{k}\right)=\inf f, \quad \lim _{k \rightarrow \infty}\left\|\operatorname{grad} f\left(p_{k}\right)\right\|=0 \quad e \quad \liminf _{k \rightarrow \infty} \Delta f\left(p_{k}\right) \geq 0 .
$$

De Peng e Terng [24], temos o seguinte lema auxiliar.

Lema 2.3 Sejam $M^{n}$ uma variedade Riemanniana conexa, $x: M^{n} \rightarrow \mathbb{Q}^{n+1}(c)$ uma imersão minima com curvatura de Gauss-Kronecker $K$ nunca nula. Para qualquer $p \in M^{n}$ fixado, é possivel escolher um referencial ortonormal $\left\{e_{1}, \ldots, e_{n+1}\right\}$, adaptado a $M^{n}$, tal que $h_{i j}=\lambda_{i} \delta_{i j}$ em $p$. Nessas condições, o Laplaciano de $F=\ln |K|$ satisfaz

$$
\Delta F=-\sum_{i, j, k=1}^{n} \frac{1}{\lambda_{i} \lambda_{j}} h_{i j ; k}^{2}-n(S-n c)
$$

onde $S=\sum_{i, j=1}^{n} h_{i j}^{2}$ é o quadrado da norma de $h$.

Demonstração: Como $K$ nunca se anula, sem perda de generalidade, podemos assumir $K>0$ e tomar $F=\ln K$. Assim, $F$ é suave e está globalmente definida em $M^{n}$. Usaremos a notação $h^{i j}$ para o elemento na posição $i j$ da matriz inversa de $\left(h_{i j}\right)$, e por isso, $h^{i j}=\frac{1}{\lambda_{i}} \delta_{i j}$ em $p$. Pela fórmula de Jacobi para matrizes invertíveis, começamos calculando $F_{k}$ :

$$
F_{k}=(\ln K)_{k}=\left(\ln \operatorname{det}\left(h_{i j}\right)\right)_{k}=\frac{1}{\operatorname{det}\left(h_{i j}\right)} \operatorname{det}\left(h_{i j}\right) \text { Traço de }\left(\left(h^{i j}\right)\left(h_{i j ; k}\right)\right)=\sum_{i, j=1}^{n} h^{i j} h_{i j ; k} .
$$

Logo, 


$$
\Delta F=\sum_{k=1}^{n} F_{k ; k}=\sum_{i, j, k=1}^{n}\left(h_{k}^{i j} h_{i j ; k}+h^{i j} h_{i j k ; k}\right)=\sum_{i, j, k=1}^{n} h_{k}^{i j} h_{i j ; k}+\sum_{i, j=1}^{n} h^{i j} \Delta h_{i j} .
$$

Como $\left(h^{i j}\right)$ é a matriz inversa de $\left(h_{i j}\right)$, temos

$$
\left(h^{i j}\right)\left(h_{i j}\right)=\mathbb{1}_{n \times n} .
$$

Derivando na direção $e_{k}$ e utilizando a regra do produto, obtemos

$$
\left(h_{k}^{i j}\right)\left(h_{i j}\right)+\left(h^{i j}\right)\left(h_{i j ; k}\right)=\mathbb{O}_{n \times n},
$$

ou seja,

$$
\left(h_{k}^{i j}\right)\left(h_{i j}\right)=-\left(h^{i j}\right)\left(h_{i j ; k}\right) .
$$

Multiplicando à direita por $\left(h^{i j}\right)$ os dois lados, chegamos a

$$
\left(h_{k}^{i j}\right)=-\left(h^{i j}\right)\left(h_{i j ; k}\right)\left(h^{i j}\right) .
$$

Desta forma, cada entrada $h_{k}^{i j}$ é dada por

$$
h_{k}^{i j}=-\sum_{l, m=1}^{n} h^{i l} h_{l m ; k} h^{m j}=-\sum_{l, m=1}^{n} h^{i l} h^{j m} h_{l m ; k} .
$$

Mas $h^{i j}=\frac{1}{\lambda_{i}} \delta_{i j}$, e portanto

$$
h_{k}^{i j}=-\sum_{l, m=1}^{n} \frac{\delta_{i l} \delta_{j m}}{\lambda_{i} \lambda_{j}} h_{l m ; k} .
$$

Logo, por (1.53), (2.2) e (2.3), temos

$$
\begin{aligned}
\Delta F & =\sum_{i, j, k=1}^{n} h_{k}^{i j} h_{i j ; k}+\sum_{i, j=1}^{n} h^{i j} \Delta h_{i j} \\
& =-\sum_{i, j, k, l, m=1}^{n} \frac{\delta_{i l} \delta_{j m}}{\lambda_{i} \lambda_{j}} h_{l m ; k} h_{i j ; k}+\sum_{i, j=1}^{n} h^{i j} h_{i j}(n c-S) \\
& =-\sum_{i, j, k=1}^{n} \frac{1}{\lambda_{i} \lambda_{j}} h_{i j ; k}^{2}+(n c-S) \sum_{i=1}^{n}\left(\sum_{j=1}^{n} h^{i j} h_{j i}\right) \\
& =-\sum_{i, j, k=1}^{n} \frac{1}{\lambda_{i} \lambda_{j}} h_{i j ; k}^{2}+(n c-S) \sum_{i=1}^{n} \delta_{i i} \\
& =-\sum_{i, j, k=1}^{n} \frac{1}{\lambda_{i} \lambda_{j}} h_{i j ; k}^{2}-n(S-n c),
\end{aligned}
$$

o que demonstra (2.1).

O lema a seguir é responsável por fornecer uma expressão para o Laplaciano de $\ln |K|$, onde $K$ é a curvatura de Gauss-Kronecker de uma variedade Riemanniana tridimensional minimamente imersa em um espaço forma quadridimensional, com curvatura seccional constante $c$. Tal lema exerce um papel central nas demonstrações dos resultados obtidos por Asperti et al. [1].

Lema 2.4 Sejam $M^{3}$ uma variedade Riemanniana conexa tridimensional, $x: M^{3} \rightarrow \mathbb{Q}^{4}(c)$ uma imersão minima com curvatura de Gauss-Kronecker $K$ nunca nula. Para qualquer $p \in M^{3}$ fixado, é possivel escolher um referencial ortonormal $\left\{e_{1}, e_{2}, e_{3}, e_{4}\right\}$, adaptado a $M^{3}$, tal que $h_{i j}=\lambda_{i} \delta_{i j}$ em p. Nessas condições, o Laplaciano de $F=\ln |K|$ satisfaz 


$$
\begin{gathered}
\Delta F=-\frac{1}{K^{2}}\left(\left(\lambda_{3}^{2} h_{22 ; 1}+\lambda_{2}^{2} h_{33 ; 1}\right)^{2}+\left(\lambda_{3}^{2} h_{11 ; 2}+\lambda_{1}^{2} h_{33 ; 2}\right)^{2}\right. \\
\left.+\left(\lambda_{2}^{2} h_{11 ; 3}+\lambda_{1}^{2} h_{22 ; 3}\right)^{2}\right)-3(S-3 c),
\end{gathered}
$$

onde $S=\sum_{i, j=1}^{3} h_{i j}^{2}$ é o quadrado da norma de $h$.

Demonstração: Por (2.1), temos

$$
\Delta F=-\sum_{i, j, k=1}^{3} \frac{1}{\lambda_{i} \lambda_{j}} h_{i j ; k}^{2}-3(S-3 c)
$$

Utilizando a Equação de Codazzi (1.41), analisando o coeficiente de $h_{12 ; 3}^{2}$ em $\sum_{i, j, k=1}^{3} \frac{1}{\lambda_{i} \lambda_{j}} h_{i j ; k}^{2}$ temos

$$
2\left(\frac{1}{\lambda_{1} \lambda_{2}}+\frac{1}{\lambda_{1} \lambda_{3}}+\frac{1}{\lambda_{2} \lambda_{3}}\right)=\frac{6 H}{K}=0
$$

Logo,

$$
\sum_{i, j, k=1}^{3} \frac{1}{\lambda_{i} \lambda_{j}} h_{i j ; k}^{2}=\sum_{i=1}^{3} \sum_{\substack{j, k=1 \\ j \neq i, k \neq i \\ j<k}}^{3}\left(\frac{1}{\lambda_{i}^{2}} h_{i i ; i}^{2}+\left(\frac{1}{\lambda_{j}^{2}}+\frac{2}{\lambda_{i} \lambda_{j}}\right) h_{j j ; i}^{2}+\left(\frac{1}{\lambda_{k}^{2}}+\frac{2}{\lambda_{i} \lambda_{k}}\right) h_{k k ; i}^{2}\right) .
$$

Sejam $i, j, k$ três índices distintos. Então, como a imersão é mínima, temos que $\lambda_{i}+\lambda_{j}=-\lambda_{k}$, $\lambda_{i}+\lambda_{k}=-\lambda_{j}$ e $h_{i i ; i}=-\left(h_{j j ; i}+h_{k k ; i}\right)$. Nessas condições,

$$
\begin{aligned}
\frac{1}{\lambda_{i}^{2}} h_{i i ; i}^{2}+\left(\frac{1}{\lambda_{j}^{2}}+\frac{2}{\lambda_{i} \lambda_{j}}\right) h_{j j ; i}^{2}+\left(\frac{1}{\lambda_{k}^{2}}+\frac{2}{\lambda_{i} \lambda_{k}}\right) h_{k k ; i}^{2} \\
=\frac{1}{\lambda_{i}^{2}}\left(h_{j j ; i}+h_{k k ; i}\right)^{2}+\left(\frac{1}{\lambda_{j}^{2}}+\frac{2}{\lambda_{i} \lambda_{j}}\right) h_{j j ; i}^{2}+\left(\frac{1}{\lambda_{k}^{2}}+\frac{2}{\lambda_{i} \lambda_{k}}\right) h_{k k ; i}^{2} \\
=\left(\frac{1}{\lambda_{i}^{2}}+\frac{2}{\lambda_{i} \lambda_{j}}+\frac{1}{\lambda_{j}^{2}}\right) h_{j j ; i}^{2}+\left(\frac{1}{\lambda_{i}^{2}}+\frac{2}{\lambda_{i} \lambda_{k}}+\frac{1}{\lambda_{k}^{2}}\right) h_{k k ; i}^{2}+\frac{2}{\lambda_{i}^{2}} h_{j j ; i} h_{k k ; i} \\
=\left(\frac{1}{\lambda_{i}}+\frac{1}{\lambda_{j}}\right)^{2} h_{j j ; i}^{2}+\left(\frac{1}{\lambda_{i}}+\frac{1}{\lambda_{k}}\right)^{2} h_{k k ; i}^{2}+\frac{2}{\lambda_{i}^{2}} h_{j j ; i} h_{k k ; i} \\
=\frac{\left(\lambda_{i}+\lambda_{j}\right)^{2}}{\lambda_{i}^{2} \lambda_{j}^{2}} h_{j j ; i}^{2}+\frac{\left(\lambda_{i}+\lambda_{k}\right)^{2}}{\lambda_{i}^{2} \lambda_{k}^{2}} h_{k k ; i}^{2}+\frac{2}{\lambda_{i}^{2}} h_{j j ; i} h_{k k ; i} \\
=\frac{\lambda_{k}^{2}}{\lambda_{i}^{2} \lambda_{j}^{2}} h_{j j ; i}^{2}+\frac{\lambda_{j}^{2}}{\lambda_{i}^{2} \lambda_{k}^{2}} h_{k k ; i}^{2}+\frac{2}{\lambda_{i}^{2}} h_{j j ; i} h_{k k ; i} \\
=\frac{1}{\lambda_{i}^{2} \lambda_{j}^{2} \lambda_{k}^{2}}\left(\lambda_{k}^{4} h_{j j ; i}^{2}+\lambda_{j}^{4} h_{k k ; i}^{2}+2 \lambda_{j}^{2} \lambda_{k}^{2} h_{j j ; i} h_{k k ; i}\right) \\
=\frac{1}{K^{2}}\left(\lambda_{k}^{2} h_{j j ; i}+\lambda_{j}^{2} h_{k k ; i}\right)^{2} .
\end{aligned}
$$

Desta forma, substituindo (2.7) em (2.6), obtemos

$$
\sum_{i, j, k=1}^{3} \frac{1}{\lambda_{i} \lambda_{j}} h_{i j ; k}^{2}=\frac{1}{K^{2}}\left(\left(\lambda_{3}^{2} h_{22 ; 1}+\lambda_{2}^{2} h_{33 ; 1}\right)^{2}+\left(\lambda_{3}^{2} h_{11 ; 2}+\lambda_{1}^{2} h_{33 ; 2}\right)^{2}+\left(\lambda_{2}^{2} h_{11 ; 3}+\lambda_{1}^{2} h_{22 ; 3}\right)^{2}\right) .
$$


Por fim, combinando (2.5) e (2.8), segue que

$$
\Delta F=-\frac{1}{K^{2}}\left(\left(\lambda_{3}^{2} h_{22 ; 1}+\lambda_{2}^{2} h_{33 ; 1}\right)^{2}+\left(\lambda_{3}^{2} h_{11 ; 2}+\lambda_{1}^{2} h_{33 ; 2}\right)^{2}+\left(\lambda_{2}^{2} h_{11 ; 3}+\lambda_{1}^{2} h_{22 ; 3}\right)^{2}\right)-3(S-3 c),
$$

o que demonstra (2.4).

Agora podemos enunciar e demonstrar três dos teoremas presentes em [1].

Teorema 2.5 Seja $M^{3}$ uma variedade Riemanniana tridimensional conexa minimamente imersa em $\mathbb{Q}^{4}(c), c \leq 0$, com curvatura de Gauss-Kronecker $K$ nunca nula. Então a função $F=\ln |K|$ é uma das seguintes opções:

(i) super-harmônica se $c=0$; ou

(ii) fortemente super-harmônica se $c<0$.

Demonstração: Por (2.4) e como $S=\sum_{i, j=1}^{3} h_{i j}^{2} \geq 0$, temos que $\Delta F \leq 9 c$. Desta forma, concluímos que

(i) se $c=0$, então $\Delta F \leq 0$, ou seja, $F$ é super-harmônica; ou

(ii) se $c<0$, então $\Delta F<0$, ou seja, $F$ é fortemente super-harmônica,

o que demonstra o afirmado no enunciado.

Teorema 2.6 Sejam $M^{3}$ uma variedade Riemanniana tridimensional conexa e $f: M^{3} \rightarrow \mathbb{Q}^{4}(c)$, $c \leq 0$, uma imersão mínima com curvatura de Gauss-Kronecker $K$ constante. Então $K$ é identicamente nula.

Demonstração: Suponha, por absurdo, que $K$ não é identicamente nula. Como $|K| \neq 0$ é constante, temos

$$
\Delta F=\Delta(\ln |K|)=0 .
$$

Por (2.4), segue que

$$
-\frac{1}{K^{2}}\left(\left(\lambda_{3}^{2} h_{22 ; 1}+\lambda_{2}^{2} h_{33 ; 1}\right)^{2}+\left(\lambda_{3}^{2} h_{11 ; 2}+\lambda_{1}^{2} h_{33 ; 2}\right)^{2}+\left(\lambda_{2}^{2} h_{11 ; 3}+\lambda_{1}^{2} h_{22 ; 3}\right)^{2}\right)-3(S-3 c)=0,
$$

ou seja,

$$
S=-\frac{1}{3 K^{2}}\left(\left(\lambda_{3}^{2} h_{22 ; 1}+\lambda_{2}^{2} h_{33 ; 1}\right)^{2}+\left(\lambda_{3}^{2} h_{11 ; 2}+\lambda_{1}^{2} h_{33 ; 2}\right)^{2}+\left(\lambda_{2}^{2} h_{11 ; 3}+\lambda_{1}^{2} h_{22 ; 3}\right)^{2}\right)+3 c .
$$

Como $c \leq 0$, obtemos que $S \leq 0$. Mas $S=\sum_{i, j=1}^{3} h_{i j}^{2} \geq 0$. Logo, concluímos que $S=0$, e chegamos que $K=0$, o que contradiz a hipótese de $K$ não ser identicamente nula, finalizando a prova.

Teorema 2.7 Seja $M^{3}$ uma variedade Riemanniana tridimensional completa minimamente imersa em $\mathbb{Q}^{4}(c), c \leq 0$, com curvatura de Ricci limitada inferiormente. Então a curvatura de GaussKronecker $K$ satisfaz $\inf |K|=0$.

Demonstração: Suponha, por absurdo, que exista $\epsilon>0$ tal que $\inf |K|=\epsilon$. Nesta situação, a função $F=\ln |K|$ está globalmente definida em $M^{3}$, é suave e limitada inferiormente. Por (2.4), obtemos

$$
\Delta F \leq-3(S-3 c)
$$

Por hipótese, a curvatura de Ricci de $M^{3}$ é limitada inferiormente e como $F$ é uma função suave e limitada inferiormente, podemos aplicar o Lema 2.2 para $F$. Desta forma, é possível obter uma sequência de pontos $\left\{p_{k}\right\}_{k \in \mathbb{N}}$ em $M^{3}$ tal que 


$$
\lim _{k \rightarrow \infty} F\left(p_{k}\right)=\inf F, \quad \lim _{k \rightarrow \infty}\left\|\operatorname{grad} F\left(p_{k}\right)\right\|=0 \quad e \quad \liminf _{k \rightarrow \infty} \Delta F\left(p_{k}\right) \geq 0 .
$$

Avaliando a inequação (2.9) em $p_{k}$, tomando o limite e utilizando (2.10), obtemos

$$
0 \leq \liminf _{k \rightarrow \infty} \Delta F\left(p_{k}\right) \leq \liminf _{k \rightarrow \infty}-3\left(S\left(p_{k}\right)-3 c\right),
$$

ou seja,

$$
-3(\inf S-3 c) \geq 0
$$

valendo,

$$
\inf S \leq 3 c \text {. }
$$

Como $c \leq 0$,

$$
\inf S \leq 0
$$

Mas $S=\sum_{i, j=1}^{3} h_{i j}^{2} \geq 0$, então

$$
\inf S \geq 0 \text {. }
$$

Combinando (2.11) e (2.12), chegamos a inf $S=0$, e concluímos que $\inf |K|=0$, o que contradiz a hipótese de que $\inf |K|=\epsilon>0$, concluindo a demonstração.

Observação 2.8 Note que nas demonstrações dos teoremas 2.5, 2.6 e 2.7, utilizamos fortemente a hipótese de $c \leq 0$ para obtermos os resultados desejados, e essa é uma das razões para separarmos os casos Euclideano e hiperbólico do caso esférico.

Existem diversos exemplos de hipersuperfícies mínimas e completas em $\mathbb{R}^{4}$ e $\mathbb{H}^{4}$ com curvatura de Gauss-Kronecker identicamente nula e que não são totalmente geodésicas. A seguir mostraremos alguns deles, que foram apresentados por Asperti et al. em [1].

Exemplo 2.9 Seja $g: M^{2} \rightarrow \mathbb{R}^{3}$ uma superfície mínima, completa e não totalmente geodésica de $\mathbb{R}^{3}$. Seja a um vetor unitário de $\mathbb{R}^{4}$ normal a $\mathbb{R}^{3}$. Desta forma, o cilindro $f: M^{2} \times \mathbb{R} \rightarrow \mathbb{R}^{4}$ dado por $f(p, t)=g(p)+t a$ é uma hipersuperfície mínima e não totalmente geodésica de $\mathbb{R}^{4}$, com curvatura de Gauss-Kronecker identicamente zero.

Um exemplo concreto desta construção pode ser dado tomando-se $x(u, v)=(v \cos u, v \operatorname{sen} u, u)$, que é uma parametrização do helicoide imerso em $\mathbb{R}^{3}$. Construindo um cilindro sobre o helicoide, obtemos uma hipersuperfície mínima e não totalmente geodésica de $\mathbb{R}^{4}$, com curvatura de GaussKronecker identicamente zero.

Exemplo 2.10 Sejam $M^{2}$ uma variedade Riemanniana bidimensional orientada e $g: M^{2} \rightarrow N^{3}$ uma imersão isométrica, onde $N^{3}$ é uma hipersuperfície umbílica de $\mathbb{H}^{4}$. Seja $\eta$ um campo de vetores normal e unitário de $N^{3}$ em $\mathbb{H}^{4}$. A aplicação $F_{g}: M^{2} \times \mathbb{R} \rightarrow \mathbb{H}^{4}$, definida por

$$
F_{g}(p, t)=\cosh t g(p)+\operatorname{senh} t \eta \circ g(p), \quad(p, t) \in M^{2} \times \mathbb{R},
$$

é chamada de suspensão de $g$ em $\mathbb{H}^{4}$. Verifica-se que quando $g$ é uma superfície mínima completa de $\mathbb{H}^{3}$, sua suspensão é uma hipersuperfície mínima e não totalmente geodésica de $\mathbb{H}^{4}$, com curvatura de Gauss-Kronecker identicamente zero.

Tomando-se $x(u, v)=(\cosh u \cosh v, \operatorname{senh} u \cos v, \operatorname{senh} u \operatorname{sen} v, \cosh u \operatorname{senh} v)$, que é uma parametrização do helicoide hiperbólico imerso em $\mathbb{H}^{3} \subset \mathbb{L}^{4}$, podemos obter um exemplo concreto da construção acima, ou seja, a suspensão do helicoide hiperbólico é uma hipersuperfície mínima e não totalmente geodésica de $\mathbb{H}^{4}$, com curvatura de Gauss-Kronecker identicamente zero. 
44 OS CASOS EUCLIDEANO $\mathbb{R}^{4}$ E HIPERBÓLICO $\mathbb{H}^{4}$ 


\section{Capítulo 3}

\section{O Caso Esférico $\mathbb{S}^{4}$}

Neste capítulo apresentamos o resultado obtido por Asperti et al. em [1], referente a imersões de hipersuperfícies tridimensionais em espaços forma quadridimensionais $\mathbb{Q}^{4}(c)$, com $c=1$.

Além do Lema 2.4 provado no capítulo anterior, precisaremos de mais dois resultados, sendo que o primeiro é uma generalização do Princípio do Máximo de Omori [23] e o segundo é um resultado puramente algébrico.

Lema 3.1 (Omori [23]) Seja $M^{n}$ uma variedade Riemanniana completa $n$-dimensional com curvaturas seccionais limitadas inferiormente e seja $f: M^{n} \rightarrow \mathbb{R}$ uma função suave que é limitada superiormente em $M^{n}$. Então existe uma sequência de pontos $\left\{p_{k}\right\}_{k \in \mathbb{N}}$ em $M^{n}$ tal que

$$
\lim _{k \rightarrow \infty} f\left(p_{k}\right)=\sup f, \quad \lim _{k \rightarrow \infty}\left\|\operatorname{grad} f\left(p_{k}\right)\right\|=0 \quad e \quad \limsup _{k \rightarrow \infty} \max _{\|X\|=1}\left\{\left(\operatorname{Hess} f\left(p_{k}\right)\right)(X, X)\right\} \leq 0 .
$$

Lema 3.2 Sejam $\lambda_{i}, i=1,2,3$, números reais tais que $3 H=\sum_{i=1}^{3} \lambda_{i}=0$ e $K=\prod_{i=1}^{3} \lambda_{i}$. Então

$$
S=\sum_{i=1}^{3} \lambda_{i}^{2} \geq 3 \sqrt[3]{2 K^{2}}
$$

e a igualdade é válida se, e somente se, pelo menos dois $\lambda_{i}$ são iguais. Além disso, vale que

$$
\sum_{i=1}^{3} \lambda_{i}^{3}=3 K
$$

Demonstração: Seja $p(\lambda)=\lambda^{3}-\frac{S}{2} \lambda-K$ o polinômio cúbico cujas raízes são $\lambda_{i}, i=1,2,3$ e defina $D=\prod_{\substack{i, j=1 \\ i<j}}^{3}\left(\lambda_{j}-\lambda_{i}\right)$. De acordo com [31] é possível escrever

$$
0 \leq D^{2}=\frac{1}{2}\left(S^{3}-54 K^{2}\right)
$$

Disso implica que $S=\sum_{i=1}^{3} \lambda_{i}^{2} \geq 3 \sqrt[3]{2 K^{2}}$ e a igualdade é válida se, e somente se, pelo menos dois $\lambda_{i}$ são iguais, demonstrando (3.1). Para provar (3.2), note que como $3 H=0$, vale $\lambda_{1}=-\left(\lambda_{2}+\lambda_{3}\right)$, $\lambda_{2}=-\left(\lambda_{1}+\lambda_{3}\right), \lambda_{3}=-\left(\lambda_{1}+\lambda_{2}\right)$. Logo

$$
\begin{aligned}
0 & =(3 H)^{3}=\left(\lambda_{1}+\lambda_{2}+\lambda_{3}\right)^{3} \\
& =\lambda_{1}^{3}+3 \lambda_{1}^{2} \lambda_{2}+3 \lambda_{1}^{2} \lambda_{3}+\lambda_{2}^{3}+3 \lambda_{2}^{2} \lambda_{1}+3 \lambda_{2}^{2} \lambda_{3}+\lambda_{3}^{3}+3 \lambda_{3}^{2} \lambda_{1}+3 \lambda_{3}^{2} \lambda_{2}+6 \lambda_{1} \lambda_{2} \lambda_{3} \\
& =\lambda_{1}^{3}+3 \lambda_{1}^{2}\left(\lambda_{2}+\lambda_{3}\right)+\lambda_{2}^{3}+3 \lambda_{2}^{2}\left(\lambda_{1}+\lambda_{3}\right)+\lambda_{3}^{3}+3 \lambda_{3}^{2}\left(\lambda_{1}+\lambda_{2}\right)+6 \lambda_{1} \lambda_{2} \lambda_{3}
\end{aligned}
$$




$$
\begin{aligned}
& =\lambda_{1}^{3}-3 \lambda_{1}^{3}+\lambda_{2}^{3}-3 \lambda_{2}^{3}+\lambda_{3}^{3}-3 \lambda_{3}^{3}+6 \lambda_{1} \lambda_{2} \lambda_{3} \\
& =-2 \lambda_{1}^{3}-2 \lambda_{2}^{3}-2 \lambda_{3}^{3}+6 \lambda_{1} \lambda_{2} \lambda_{3},
\end{aligned}
$$

e portanto,

$$
\sum_{i=1}^{3} \lambda_{i}^{3}=\lambda_{1}^{3}+\lambda_{2}^{3}+\lambda_{3}^{3}=3 \lambda_{1} \lambda_{2} \lambda_{3}=3 K
$$

Agora podemos enunciar e demonstrar o teorema presente em [1].

Teorema 3.3 Seja $f: M^{3} \rightarrow \mathbb{S}^{4}$ uma imersão mínima de uma variedade Riemanniana completa com curvatura de Gauss-Kronecker constante $K \neq 0$. Então $f\left(M^{3}\right)$ é isométrico ao toro de Clifford $\mathbb{S}^{1}\left(\frac{\sqrt{3}}{3}\right) \times \mathbb{S}^{2}\left(\frac{\sqrt{6}}{3}\right)$.

Demonstração: Começamos mostrando que a função $S$ é limitada $(0<S \leq 3)$, para podermos aplicar o Lema 3.1 para $S$. Como $K$ é constante, temos

$$
\Delta F=\Delta(\ln |K|)=0 .
$$

Por (2.4) e pelo fato de $K \neq 0$ e de $c=1$, segue que

$$
-\frac{1}{K^{2}}\left(\left(\lambda_{3}^{2} h_{22 ; 1}+\lambda_{2}^{2} h_{33 ; 1}\right)^{2}+\left(\lambda_{3}^{2} h_{11 ; 2}+\lambda_{1}^{2} h_{33 ; 2}\right)^{2}+\left(\lambda_{2}^{2} h_{11 ; 3}+\lambda_{1}^{2} h_{22 ; 3}\right)^{2}\right)-3(S-3)=0 .
$$

Isolando $S$, obtemos

$$
S=-\frac{1}{3 K^{2}}\left(\left(\lambda_{3}^{2} h_{22 ; 1}+\lambda_{2}^{2} h_{33 ; 1}\right)^{2}+\left(\lambda_{3}^{2} h_{11 ; 2}+\lambda_{1}^{2} h_{33 ; 2}\right)^{2}+\left(\lambda_{2}^{2} h_{11 ; 3}+\lambda_{1}^{2} h_{22 ; 3}\right)^{2}\right)+3,
$$

ou seja,

$$
S \leq 3
$$

Note que $S=\sum_{i, j=1}^{3} h_{i j}^{2}>0$, pois, caso contrário, $K=0$. Por (3.4) obtemos

$$
0<S \leq 3
$$

Definimos agora $f_{3}: M^{3} \rightarrow \mathbb{R}$ por

$$
f_{3}=\sum_{i=1}^{3} \lambda_{i}^{3}=\text { Traço de }\left(h_{i j}\right)^{3}=\sum_{i, j, k=1}^{3} h_{i j} h_{j k} h_{k i} .
$$

Como a imersão é mínima e $K$ é constante, por (3.2), temos que $f_{3}=3 K$, e obtemos as seguintes condições de curvatura

$$
\begin{gathered}
\sum_{i=1}^{3} h_{i i}=3 H=0, \\
\sum_{i, j=1}^{3} h_{i j}^{2}=S, \\
\sum_{i, j, k=1}^{3} h_{i j} h_{j k} h_{k i}=f_{3}=3 K .
\end{gathered}
$$

Diferenciando (3.6) em relação a $e_{k}$, obtemos 


$$
0=3 H_{k}=\sum_{i=1}^{3} h_{i i ; k}
$$

Diferenciando (3.9) novamente em relação a $e_{k}$, obtemos

$$
0=3 H_{k ; k}=\sum_{i=1}^{3} h_{i i k ; k} .
$$

Diferenciando (3.7) em relação a $e_{k}$, obtemos

$$
S_{k}=2 \sum_{i, j=1}^{3} h_{i j} h_{i j ; k} .
$$

Diferenciando (3.11) novamente em relação a $e_{k}$, obtemos

$$
S_{k ; k}=2 \sum_{i, j=1}^{3}\left(h_{i j ; k}^{2}+h_{i j} h_{i j k ; k}\right) .
$$

Como $h_{i j}=\lambda_{i} \delta_{i j}$, de (3.11) e (3.12), chegamos a

$$
S_{k}=2 \sum_{i, j=1}^{3} h_{i j} h_{i j ; k}=2 \sum_{i, j=1}^{3} \lambda_{i} \delta_{i j} h_{i j ; k}=2 \sum_{i=1}^{3} \lambda_{i} h_{i i ; k},
$$

e

$$
\begin{aligned}
S_{k ; k} & =2 \sum_{i, j=1}^{3}\left(h_{i j ; k}^{2}+h_{i j} h_{i j k ; k}\right)=2 \sum_{i, j=1}^{3}\left(h_{i j ; k}^{2}+\lambda_{i} \delta_{i j} h_{i j k ; k}\right) \\
& =2 \sum_{i, j=1}^{3} h_{i j ; k}^{2}+2 \sum_{i=1}^{3} \lambda_{i} h_{i i k ; k} .
\end{aligned}
$$

Diferenciando (3.8) em relação a $e_{l}$, obtemos

$$
\begin{aligned}
0 & =(3 K)_{l}=\left(\sum_{i, j, k=1}^{3} h_{i j} h_{j k} h_{k i}\right)_{l} \\
& =\sum_{i, j, k=1}^{3}\left(h_{i j ; l} h_{j k} h_{k i}+h_{i j} h_{j k ; l} h_{k i}+h_{i j} h_{j k} h_{k i ; l}\right) .
\end{aligned}
$$

Logo, utilizando (1.41) e renomeando índices, obtemos

$$
3 \sum_{i, j, k=1}^{3} h_{i k} h_{j k} h_{i j ; l}=0
$$

ou seja,

$$
\sum_{i, j, k=1}^{3} h_{i k} h_{j k} h_{i j ; l}=0 .
$$

Diferenciando (3.15) novamente em relação a $e_{l}$, obtemos 


$$
\begin{aligned}
0 & =\left(\sum_{i, j, k=1}^{3} h_{i k} h_{j k} h_{i j ; l}\right)_{l} \\
& =\sum_{i, j, k=1}^{3}\left(h_{i k ; l} h_{j k} h_{i j ; l}+h_{i k} h_{j k ; l} h_{i j ; l}+h_{i k} h_{j k} h_{i j l ; l}\right) .
\end{aligned}
$$

Como $h_{i j}=\lambda_{i} \delta_{i j}$, de (3.15) e (3.16), chegamos a

$$
0=\sum_{i, j, k=1}^{3} h_{i k} h_{j k} h_{i j ; l}=\sum_{i, j, k=1}^{3} \lambda_{i} \delta_{i k} \lambda_{j} \delta_{j k} h_{i j ; l}=\sum_{i=1}^{3} \lambda_{i}^{2} h_{i i ; l},
$$

e

$$
\begin{aligned}
0 & =\sum_{i, j, k=1}^{3}\left(h_{i k ; l} h_{j k} h_{i j ; l}+h_{i k} h_{j k ; l} h_{i j ; l}+h_{i k} h_{j k} h_{i j l ; l}\right) \\
& =\sum_{i, j, k=1}^{3}\left(h_{i k ; l} \lambda_{j} \delta_{j k} h_{i j ; l}+\lambda_{i} \delta_{i k} h_{j k ; l} h_{i j ; l}+\lambda_{i} \delta_{i k} \lambda_{j} \delta_{j k} h_{i j l ; l}\right),
\end{aligned}
$$

ou seja,

$$
\sum_{i=1}^{3} \lambda_{i}^{2} h_{i i l ; l}=-2 \sum_{i, j=1}^{3} \lambda_{i} h_{i j ; l}^{2}
$$

Assim, combinando (3.9), (3.13) e (3.17), obtemos o seguinte sistema linear

$$
\left\{\begin{array}{l}
\sum_{i=1}^{3} h_{i i ; j}=0 \\
\sum_{i=1}^{3} \lambda_{i} h_{i i ; j}=\frac{1}{2} S_{j} \\
\sum_{i=1}^{3} \lambda_{i}^{2} h_{i i ; j}=0
\end{array}\right.
$$

Combinando (3.10), (3.14) e (3.18), obtemos o seguinte sistema linear

$$
\left\{\begin{array}{l}
\sum_{i=1}^{3} h_{i i l ; l}=0 \\
\sum_{i=1}^{3} \lambda_{i} h_{i i l ; l}=-\sum_{i, j=1}^{3} h_{i j ; l}^{2}+\frac{1}{2} S_{l ; l} ; \\
\sum_{i=1}^{3} \lambda_{i}^{2} h_{i i l ; l}=-2 \sum_{i, j=1}^{3} \lambda_{i} h_{i j ; l}^{2} .
\end{array}\right.
$$

Por (1.39), temos que a curvatura escalar $R$ de $M^{3}$ vale

$$
R=3(3-1) 1-S=6-S,
$$

e, como $S$ é limitada, $R$ também o é. Desta forma, vemos que as curvaturas seccionais de $M^{3}$ são limitadas inferiormente e podemos aplicar o Lema 3.1 para obter uma sequência $\left\{p_{k}\right\}_{k \in \mathbb{N}}$ de pontos em $M^{3}$ tal que 


$$
\lim _{k \rightarrow \infty} S\left(p_{k}\right)=\sup S, \quad \lim _{k \rightarrow \infty}\left\|\operatorname{grad} S\left(p_{k}\right)\right\|=0 \quad e \quad \limsup _{k \rightarrow \infty} \max _{\|X\|=1}\left\{\left(\operatorname{Hess} S\left(p_{k}\right)\right)(X, X)\right\} \leq 0 .
$$

Além disso, como $S$ é limitada, tomando uma subsequência se necessário, podemos assumir também que

$$
\lim _{k \rightarrow \infty} \lambda_{i}\left(p_{k}\right)=\tilde{\lambda}_{i}, \quad i=1,2,3
$$

Note que existem três possibilidades para os valores $\tilde{\lambda}_{i}$ :

(i) todos os $\tilde{\lambda}_{i}$ são iguais; ou

(ii) existem apenas dois índices $i, j \in\{1,2,3\}, i \neq j$, tais que $\tilde{\lambda}_{i}=\tilde{\lambda}_{j}$; ou

(iii) todos os $\tilde{\lambda}_{i}$ são distintos.

Se supomos que todos os $\tilde{\lambda}_{i}$ são iguais e valem $\tilde{\lambda}$, como a imersão é mínima, temos

$$
0=\lim _{k \rightarrow \infty} H\left(p_{k}\right)=\lim _{k \rightarrow \infty} \sum_{i=1}^{3} \lambda_{i}\left(p_{k}\right)=\sum_{i=1}^{3} \lim _{k \rightarrow \infty} \lambda_{i}\left(p_{k}\right)=\sum_{i=1}^{3} \tilde{\lambda}_{i}=3 \tilde{\lambda},
$$

ou seja,

$$
\tilde{\lambda}=0
$$

o que é um absurdo, já que isto implicaria que $K=0$.

De (3.1) segue que $3 \sqrt[3]{2 K^{2}}$ é um limitante inferior para $S$ e, por isso, temos

$$
3 \sqrt[3]{2 K^{2}} \leq \inf S \leq \sup S
$$

Supondo $\tilde{\lambda}_{i}=\tilde{\lambda}_{j}$, com $i \neq j$, mostraremos que $S$, além de limitada, é constante. Por (3.1) e (3.22), obtemos

$$
\sup S=3 \sqrt[3]{2 K^{2}}
$$

Combinando (3.23) com (3.24) chegamos a

$$
3 \sqrt[3]{2 K^{2}} \leq \inf S \leq \sup S=3 \sqrt[3]{2 K^{2}}
$$

ou seja,

$$
\inf S=\sup S,
$$

e concluímos que $S$ é constante. Por (1.54), temos

$$
0=\frac{1}{2} \Delta S \geq S(3-S)
$$

ou seja,

$$
S(S-3) \geq 0 .
$$

Mas, como visto em (3.5), $0<S \leq 3$. Então concluímos que $S=3$ e segue, pela versão local do resultado clássico de Chern et al. [6], que a imagem de $M^{3}$ está contida no toro de Clifford $\mathbb{S}^{1}\left(\frac{\sqrt{3}}{3}\right) \times \mathbb{S}^{2}\left(\frac{\sqrt{6}}{3}\right)$. Como $M^{3}$ é completa, $f\left(M^{3}\right)$ é isométrico ao toro.

Mostraremos que se supusermos $\tilde{\lambda}_{i} \neq \tilde{\lambda}_{j}$, para $i \neq j$ chegaremos a uma contradição. De fato, se isto ocorre, então existe um número natural $N>0$ tal que $\lambda_{i}\left(p_{k}\right) \neq \lambda_{j}\left(p_{k}\right)$, para $i \neq j$ e $k>N$. Variando-se o índice $i$ de 1 a 3 , em (3.19), obtemos

$$
\left\{\begin{array}{l}
h_{11 ; j}+h_{22 ; j}+h_{33 ; j}=0 ; \\
\lambda_{1} h_{11 ; j}+\lambda_{2} h_{22 ; j}+\lambda_{3} h_{33 ; j}=\frac{1}{2} S_{j} \\
\lambda_{1}^{2} h_{11 ; j}+\lambda_{2}^{2} h_{22 ; j}+\lambda_{3}^{2} h_{33 ; j}=0 .
\end{array}\right.
$$


Analisando as soluções de (3.25), mostraremos que $\lim _{k \rightarrow \infty} h_{i j ; l}\left(p_{k}\right)=0$, para qualquer combinação de índices $i, j, l$. Substituindo a segunda equação de (3.25) pela soma dela com o resultado da multiplicação da primeira equação por $-\lambda_{1}$, e substituindo a terceira equação pela soma dela com o resultado da multiplicação da primeira equação por $-\lambda_{1}^{2}$, obtemos

$$
\left\{\begin{array}{l}
h_{11 ; j}+h_{22 ; j}+h_{33 ; j}=0 ; \\
\left(\lambda_{2}-\lambda_{1}\right) h_{22 ; j}+\left(\lambda_{3}-\lambda_{1}\right) h_{33 ; j}=\frac{1}{2} S_{j} \\
\left(\lambda_{2}^{2}-\lambda_{1}^{2}\right) h_{22 ; j}+\left(\lambda_{3}^{2}-\lambda_{1}^{2}\right) h_{33 ; j}=0 .
\end{array}\right.
$$

Substituindo a terceira equação de (3.26) pela soma dela com o resultado da multiplicação da segunda equação por $-\left(\lambda_{2}+\lambda_{1}\right)$, obtemos

$$
\left\{\begin{array}{l}
h_{11 ; j}+h_{22 ; j}+h_{33 ; j}=0 ; \\
\left(\lambda_{2}-\lambda_{1}\right) h_{22 ; j}+\left(\lambda_{3}-\lambda_{1}\right) h_{33 ; j}=\frac{1}{2} S_{j} ; \\
\left(\lambda_{3}^{2}-\lambda_{1}^{2}-\left(\lambda_{3}-\lambda_{1}\right)\left(\lambda_{2}+\lambda_{1}\right)\right) h_{33 ; j}=-\frac{\lambda_{2}+\lambda_{1}}{2} S_{j} .
\end{array}\right.
$$

Pela terceira equação de (3.27), temos

$$
-\frac{\lambda_{2}+\lambda_{1}}{2} S_{j}=\left(\lambda_{3}^{2}-\lambda_{1}^{2}-\left(\lambda_{3}-\lambda_{1}\right)\left(\lambda_{2}+\lambda_{1}\right)\right) h_{33 ; j}=\left(\lambda_{3}-\lambda_{1}\right)\left(\lambda_{3}-\lambda_{2}\right) h_{33 ; j} .
$$

Utilizando que $\lambda_{3}=-\left(\lambda_{1}+\lambda_{2}\right)$,

$$
h_{33 ; j}=-\frac{\lambda_{1}+\lambda_{2}}{2\left(\lambda_{3}-\lambda_{1}\right)\left(\lambda_{3}-\lambda_{2}\right)} S_{j}=\frac{\lambda_{3}}{2\left(\lambda_{3}-\lambda_{1}\right)\left(\lambda_{3}-\lambda_{2}\right)} S_{j} .
$$

Substituindo (3.28) na segunda equação de (3.27), obtemos

$$
\left(\lambda_{2}-\lambda_{1}\right) h_{22 ; j}+\left(\lambda_{3}-\lambda_{1}\right) \frac{\lambda_{3}}{2\left(\lambda_{3}-\lambda_{1}\right)\left(\lambda_{3}-\lambda_{2}\right)} S_{j}=\frac{1}{2} S_{j},
$$

ou seja,

$$
\left(\lambda_{2}-\lambda_{1}\right) h_{22 ; j}=\frac{1}{2} S_{j}-\frac{\lambda_{3}}{2\left(\lambda_{3}-\lambda_{2}\right)} S_{j}=\frac{\lambda_{3}-\lambda_{2}-\lambda_{3}}{2\left(\lambda_{3}-\lambda_{2}\right)} S_{j}=-\frac{\lambda_{2}}{2\left(\lambda_{3}-\lambda_{2}\right)} S_{j} .
$$

Logo,

$$
h_{22 ; j}=\frac{\lambda_{2}}{2\left(\lambda_{2}-\lambda_{1}\right)\left(\lambda_{2}-\lambda_{3}\right)} S_{j} .
$$

Por fim, substituindo (3.28) e (3.29) na primeira equação de (3.27), obtemos

$$
\begin{aligned}
h_{11 ; j} & =-\left(h_{22 ; j}+h_{33 ; j}\right) \\
& =-\left(\frac{\lambda_{2}}{2\left(\lambda_{2}-\lambda_{1}\right)\left(\lambda_{2}-\lambda_{3}\right)} S_{j}+\frac{\lambda_{3}}{2\left(\lambda_{3}-\lambda_{1}\right)\left(\lambda_{3}-\lambda_{2}\right)} S_{j}\right) \\
& =-\frac{\lambda_{2}\left(\lambda_{3}-\lambda_{1}\right)-\lambda_{3}\left(\lambda_{2}-\lambda_{1}\right)}{2\left(\lambda_{2}-\lambda_{1}\right)\left(\lambda_{2}-\lambda_{3}\right)\left(\lambda_{3}-\lambda_{1}\right)} S_{j} \\
& =\frac{\lambda_{1}\left(\lambda_{2}-\lambda_{3}\right)}{2\left(\lambda_{2}-\lambda_{1}\right)\left(\lambda_{2}-\lambda_{3}\right)\left(\lambda_{3}-\lambda_{1}\right)} S_{j} \\
& =\frac{\lambda_{1}}{2\left(\lambda_{1}-\lambda_{2}\right)\left(\lambda_{1}-\lambda_{3}\right)} S_{j} .
\end{aligned}
$$


Combinando (3.28), (3.29) e (3.30), obtemos o seguinte sistema linear

$$
\left\{\begin{aligned}
h_{11 ; j} & =\frac{\lambda_{1}}{2\left(\lambda_{1}-\lambda_{2}\right)\left(\lambda_{1}-\lambda_{3}\right)} S_{j} \\
h_{22 ; j} & =\frac{\lambda_{2}}{2\left(\lambda_{2}-\lambda_{1}\right)\left(\lambda_{2}-\lambda_{3}\right)} S_{j} \\
h_{33 ; j} & =\frac{\lambda_{3}}{2\left(\lambda_{3}-\lambda_{1}\right)\left(\lambda_{3}-\lambda_{2}\right)} S_{j},
\end{aligned}\right.
$$

ou seja,

$$
h_{i i ; j}=\frac{\lambda_{i}}{2}\left(\prod_{\substack{l=1 \\ l \neq i}}^{3} \frac{1}{\lambda_{i}-\lambda_{l}}\right) S_{j} .
$$

Por $(3.21)$, temos

$$
0=\lim _{k \rightarrow \infty}\left\|\operatorname{grad} S\left(p_{k}\right)\right\|=\lim _{k \rightarrow \infty}\left\|\sum_{i=1}^{3} S_{i}\left(p_{k}\right) e_{i}\right\|,
$$

ou seja,

$$
\lim _{k \rightarrow \infty} S_{i}\left(p_{k}\right)=0, \quad \forall i=1,2,3 .
$$

Avaliando (3.31) em $p_{k}$, tomando o limite para $k \rightarrow \infty$, utilizando (3.22) e (3.32), obtemos

$$
\begin{aligned}
\lim _{k \rightarrow \infty} h_{i i ; j}\left(p_{k}\right) & =\lim _{k \rightarrow \infty} \frac{\lambda_{i}\left(p_{k}\right)}{2}\left(\prod_{\substack{l=1 \\
l \neq i}}^{3} \frac{1}{\lambda_{i}\left(p_{k}\right)-\lambda_{l}\left(p_{k}\right)}\right) S_{j}\left(p_{k}\right) \\
& =\frac{\tilde{\lambda}_{i}}{2}\left(\prod_{\substack{l=1 \\
l \neq i}}^{3} \frac{1}{\tilde{\lambda}_{i}-\tilde{\lambda}_{l}}\right) \lim _{k \rightarrow \infty} S_{j}\left(p_{k}\right) \\
& =0 .
\end{aligned}
$$

Avaliando (3.3) em $p_{k}$, tomando o limite para $k \rightarrow \infty$, utilizando (3.21) e (3.33), obtemos

$$
\sup S=3 .
$$

Por (3.21), temos

$$
\limsup _{k \rightarrow \infty} \max _{\|X\|=1}\left\{\left(\operatorname{Hess} S\left(p_{k}\right)\right)(X, X)\right\} \leq 0
$$

Logo, em particular, vale

$$
\limsup _{k \rightarrow \infty} S_{i ; i}\left(p_{k}\right)=\limsup _{k \rightarrow \infty}\left(\operatorname{Hess} S\left(p_{k}\right)\right)\left(e_{i}, e_{i}\right) \leq 0, \quad \forall i=1,2,3,
$$

e, portanto,

$$
\limsup _{k \rightarrow \infty} \Delta S\left(p_{k}\right)=\limsup _{k \rightarrow \infty} \sum_{i=1}^{3} S_{i ; i}\left(p_{k}\right) \leq 0 .
$$

Note que, utilizando (1.41), vale

$$
\sum_{i, j, k=1}^{3} h_{i j ; k}^{2}=\sum_{i=1}^{3} h_{i i ; i}^{2}+3 \sum_{\substack{i, j=1 \\ j \neq i}}^{3} h_{i i ; j}^{2}+6 h_{12 ; 3}^{2} .
$$


Substituindo (3.36) em (1.54), temos

$$
\frac{1}{2} \Delta S=(3-S) S+\sum_{i, j, k=1}^{3} h_{i j ; k}^{2}=(3-S) S+\sum_{i=1}^{3} h_{i i ; i}^{2}+3 \sum_{\substack{i, j=1 \\ j \neq i}}^{3} h_{i i ; j}^{2}+6 h_{12 ; 3}^{2},
$$

ou seja,

$$
h_{12 ; 3}^{2}=\frac{1}{6}\left(\frac{1}{2} \Delta S+(S-3) S-\sum_{i=1}^{3} h_{i i ; i}^{2}-3 \sum_{\substack{i, j=1 \\ j \neq i}}^{3} h_{i i ; j}^{2}\right) .
$$

Avaliando (3.37) em $p_{k}$, tomando o limite para $k \rightarrow \infty$, utilizando (3.21), (3.33), (3.34) e (3.35), obtemos

$$
\limsup _{k \rightarrow \infty} h_{12 ; 3}^{2}\left(p_{k}\right)=\frac{1}{12} \limsup _{k \rightarrow \infty} \Delta S\left(p_{k}\right) \leq 0 .
$$

Mas $h_{12 ; 3}^{2} \geq 0$. Então

$$
\lim _{k \rightarrow \infty} h_{12 ; 3}^{2}\left(p_{k}\right)=0,
$$

e,

$$
\lim _{k \rightarrow \infty} h_{12 ; 3}\left(p_{k}\right)=0 .
$$

Desta forma, combinando (3.33) e (3.38), temos

$$
\lim _{k \rightarrow \infty} h_{i j ; l}\left(p_{k}\right)=0, \quad \forall i, j, l=1,2,3 .
$$

Além disso, avaliando (1.54) em $p_{k}$, tomando o limite para $k \rightarrow \infty$, utilizando (3.21), (3.34) e (3.39), obtemos

$$
\lim _{k \rightarrow \infty} \Delta S\left(p_{k}\right)=0
$$

e,

$$
\lim _{k \rightarrow \infty} S_{i ; i}\left(p_{k}\right)=0, \quad \forall i=1,2,3 .
$$

Variando-se o índice $i$ de 1 a 3 , em (3.20), obtemos

$$
\left\{\begin{array}{l}
h_{11 l ; l}+h_{22 l ; l}+h_{33 l ; l}=0 ; \\
\lambda_{1} h_{11 l ; l}+\lambda_{2} h_{22 l ; l}+\lambda_{3} h_{33 l ; l}=A \\
\lambda_{1}^{2} h_{11 l ; l}+\lambda_{2}^{2} h_{22 l ; l}+\lambda_{3}^{2} h_{33 l ; l}=B
\end{array}\right.
$$

onde

$$
\left\{\begin{array}{l}
A=-\sum_{i, j=1}^{3} h_{i j ; l}^{2}+\frac{1}{2} S_{l ; l} \\
B=-2 \sum_{i, j=1}^{3} \lambda_{i} h_{i j ; l}^{2} .
\end{array}\right.
$$

Analisando as soluções de (3.41), mostraremos que $\lim _{k \rightarrow \infty} h_{i i l ; l}\left(p_{k}\right)=0$, para qualquer combinação de índices $i, l$. Substituindo a segunda equação de (3.41) pela soma dela com o resultado da multiplicação da primeira equação por $-\lambda_{1}$, e substituindo a terceira equação pela soma dela com o resultado da multiplicação da primeira equação por $-\lambda_{1}^{2}$, obtemos 


$$
\left\{\begin{array}{l}
h_{11 l ; l}+h_{22 l ; l}+h_{33 l ; l}=0 ; \\
\left(\lambda_{2}-\lambda_{1}\right) h_{22 l ; l}+\left(\lambda_{3}-\lambda_{1}\right) h_{33 l ; l}=A ; \\
\left(\lambda_{2}^{2}-\lambda_{1}^{2}\right) h_{22 l ; l}+\left(\lambda_{3}^{2}-\lambda_{1}^{2}\right) h_{33 l ; l}=B .
\end{array}\right.
$$

Substituindo a terceira equação de (3.43) pela soma dela com o resultado da multiplicação da segunda equação por $-\left(\lambda_{2}+\lambda_{1}\right)$, obtemos

$$
\left\{\begin{array}{l}
h_{11 l ; l}+h_{22 l ; l}+h_{33 l ; l}=0 \\
\left(\lambda_{2}-\lambda_{1}\right) h_{22 l ; l}+\left(\lambda_{3}-\lambda_{1}\right) h_{33 l ; l}=A ; \\
\left(\lambda_{3}^{2}-\lambda_{1}^{2}-\left(\lambda_{3}-\lambda_{1}\right)\left(\lambda_{2}+\lambda_{1}\right)\right) h_{33 l ; l}=B-\left(\lambda_{2}+\lambda_{1}\right) A .
\end{array}\right.
$$

Pela terceira equação de (3.44), temos

$$
B-\left(\lambda_{2}+\lambda_{1}\right) A=\left(\lambda_{3}^{2}-\lambda_{1}^{2}-\left(\lambda_{3}-\lambda_{1}\right)\left(\lambda_{2}+\lambda_{1}\right)\right) h_{33 l ; l}=\left(\lambda_{3}-\lambda_{1}\right)\left(\lambda_{3}-\lambda_{2}\right) h_{33 l ; l},
$$

ou seja,

$$
h_{33 l ; l}=\frac{B-\left(\lambda_{1}+\lambda_{2}\right) A}{\left(\lambda_{3}-\lambda_{1}\right)\left(\lambda_{3}-\lambda_{2}\right)} .
$$

Substituindo (3.45) na segunda equação de (3.44), obtemos

$$
\left(\lambda_{2}-\lambda_{1}\right) h_{22 l ; l}+\left(\lambda_{3}-\lambda_{1}\right) \frac{B-\left(\lambda_{1}+\lambda_{2}\right) A}{\left(\lambda_{3}-\lambda_{1}\right)\left(\lambda_{3}-\lambda_{2}\right)}=A,
$$

ou seja,

$$
\begin{aligned}
\left(\lambda_{2}-\lambda_{1}\right) h_{22 l ; l} & =A-\frac{B-\left(\lambda_{1}+\lambda_{2}\right) A}{\lambda_{3}-\lambda_{2}} \\
& =\frac{\left(\lambda_{3}-\lambda_{2}\right) A-B+\left(\lambda_{1}+\lambda_{2}\right) A}{\lambda_{3}-\lambda_{2}} \\
& =\frac{B-\left(\lambda_{1}+\lambda_{3}\right) A}{\lambda_{2}-\lambda_{3}} .
\end{aligned}
$$

Logo,

$$
h_{22 l ; l}=\frac{B-\left(\lambda_{1}+\lambda_{3}\right) A}{\left(\lambda_{2}-\lambda_{1}\right)\left(\lambda_{2}-\lambda_{3}\right)} .
$$

Por fim, substituindo (3.45) e (3.46) na primeira equação de (3.44), obtemos

$$
\begin{aligned}
h_{11 l ; l} & =-\left(h_{22 l ; l}+h_{33 l ; l}\right) \\
& =-\left(\frac{B-\left(\lambda_{1}+\lambda_{3}\right) A}{\left(\lambda_{2}-\lambda_{1}\right)\left(\lambda_{2}-\lambda_{3}\right)}+\frac{B-\left(\lambda_{1}+\lambda_{2}\right) A}{\left(\lambda_{3}-\lambda_{1}\right)\left(\lambda_{3}-\lambda_{2}\right)}\right) \\
& =-\frac{\left(\lambda_{3}-\lambda_{1}\right)\left(B-\left(\lambda_{1}+\lambda_{3}\right) A\right)-\left(\lambda_{2}-\lambda_{1}\right)\left(B-\left(\lambda_{1}+\lambda_{2}\right) A\right)}{\left(\lambda_{2}-\lambda_{1}\right)\left(\lambda_{2}-\lambda_{3}\right)\left(\lambda_{3}-\lambda_{1}\right)} \\
& =-\frac{\left(\lambda_{3}-\lambda_{2}\right)\left(B-\left(\lambda_{2}+\lambda_{3}\right) A\right)}{\left(\lambda_{2}-\lambda_{1}\right)\left(\lambda_{2}-\lambda_{3}\right)\left(\lambda_{3}-\lambda_{1}\right)} \\
& =\frac{B-\left(\lambda_{2}+\lambda_{3}\right) A}{\left(\lambda_{1}-\lambda_{2}\right)\left(\lambda_{1}-\lambda_{3}\right)} .
\end{aligned}
$$

Combinando (3.45), (3.46) e (3.47), obtemos o seguinte sistema linear 


$$
\left\{\begin{aligned}
h_{11 l ; l} & =\frac{B-\left(\lambda_{2}+\lambda_{3}\right) A}{\left(\lambda_{1}-\lambda_{2}\right)\left(\lambda_{1}-\lambda_{3}\right)} \\
h_{22 l ; l}= & \frac{B-\left(\lambda_{1}+\lambda_{3}\right) A}{\left(\lambda_{2}-\lambda_{1}\right)\left(\lambda_{2}-\lambda_{3}\right)} \\
h_{33 l ; l}= & \frac{B-\left(\lambda_{1}+\lambda_{2}\right) A}{\left(\lambda_{3}-\lambda_{1}\right)\left(\lambda_{3}-\lambda_{2}\right)},
\end{aligned}\right.
$$

ou seja,

$$
h_{i i l ; l}=\left(B-A \sum_{\substack{j=1 \\ j \neq i}}^{3} \lambda_{j}\right) \prod_{\substack{m=1 \\ m \neq i}}^{3} \frac{1}{\lambda_{i}-\lambda_{m}} .
$$

Avaliando (3.42) em $p_{k}$, tomando o limite para $k \rightarrow \infty$, utilizando (3.22), (3.39) e (3.40), obtemos

$$
\lim _{k \rightarrow \infty} A\left(p_{k}\right)=\lim _{k \rightarrow \infty}\left(-\sum_{i, j=1}^{3} h_{i j ; l}^{2}\left(p_{k}\right)+\frac{1}{2} S_{l ; l}\left(p_{k}\right)\right)=0
$$

$\mathrm{e}$

$$
\lim _{k \rightarrow \infty} B\left(p_{k}\right)=\lim _{k \rightarrow \infty}-2 \sum_{i, j=1}^{3} \lambda_{i}\left(p_{k}\right) h_{i j ; l}^{2}\left(p_{k}\right)=0 .
$$

Desta forma, avaliando (3.48) em $p_{k}$, tomando o limite para $k \rightarrow \infty$, utilizando (3.22), (3.49) e (3.50), obtemos

$$
\lim _{k \rightarrow \infty} h_{i i l ; l}\left(p_{k}\right)=\lim _{k \rightarrow \infty}\left(\left(B\left(p_{k}\right)-A\left(p_{k}\right) \sum_{\substack{j=1 \\ j \neq i}}^{3} \lambda_{j}\left(p_{k}\right)\right) \prod_{\substack{m=1 \\ m \neq i}}^{3} \frac{1}{\lambda_{i}\left(p_{k}\right)-\lambda_{m}\left(p_{k}\right)}\right)=0,
$$

para qualquer combinação de índices $i, l$.

Com as informações coletadas mostraremos que chegamos a um absurdo, e por isso podemos descartar a possibilidade de todos os $\tilde{\lambda}_{i}$ serem distintos. Por (1.50) e (1.52), temos

$$
h_{i i j ; j}-h_{j j i ; i}=h_{i j i ; j}-h_{j i j ; i}=\left(\lambda_{i}-\lambda_{j}\right)\left(1+\lambda_{i} \lambda_{j}\right) .
$$

Desta forma, avaliando (3.52) em $p_{k}$ para todo $i \neq j$, tomando o limite para $k \rightarrow \infty$, utilizando (3.22) e (3.51), obtemos

$$
\left(\tilde{\lambda}_{i}-\tilde{\lambda}_{j}\right)\left(1+\tilde{\lambda}_{i} \tilde{\lambda}_{j}\right)=0 .
$$

Como estamos supondo que $\tilde{\lambda}_{i} \neq \tilde{\lambda}_{j}$ para $i \neq j$, de $(3.53)$ temos que $1+\tilde{\lambda}_{i} \tilde{\lambda}_{j}=0$ para $i \neq j$. Desta forma, chegamos ao seguinte sistema

$$
\left\{\begin{array}{l}
1+\tilde{\lambda}_{1} \tilde{\lambda}_{2}=0 \\
1+\tilde{\lambda}_{1} \tilde{\lambda}_{3}=0 \\
1+\tilde{\lambda}_{2} \tilde{\lambda}_{3}=0
\end{array}\right.
$$

Isolando $\tilde{\lambda}_{1}$ na primeira equação de (3.54) e substituindo na segunda, chegamos a 


$$
\left\{\begin{array}{l}
\tilde{\lambda}_{1}=-\frac{1}{\tilde{\lambda}_{2}} ; \\
1-\frac{\tilde{\lambda}_{3}}{\tilde{\lambda}_{2}}=0 ; \\
1+\tilde{\lambda}_{2} \tilde{\lambda}_{3}=0 .
\end{array}\right.
$$

Isolando $\tilde{\lambda}_{3}$ na segunda equação de (3.55), temos que

$$
\tilde{\lambda}_{3}=\tilde{\lambda}_{2},
$$

o que é um absurdo, finalizando a demonstração. 
O CASO ESFÉRICO $\mathbb{S}^{4}$ 


\section{Capítulo 4}

\section{Uma Classificação de Hipersuperfícies Mínimas de $\mathbb{Q}^{4}(c)$}

Neste capítulo apresentamos dois teoremas que são obtidos combinando os resultados de Asperti et al. [1], mais especificamente o Teorema 2.6 e o Teorema 3.3, com os resultados de Hasanis et al. [17, 18, 19]. Optamos por realizar também um estudo aprofundado do item (i) do Teorema 4.1, presente em [17], e destacamos que as demonstrações para o item (ii) do Teorema 4.1 e para o item (ii) do Teorema 4.2 podem ser encontradas, respectivamente, em [18] e [19].

Teorema 4.1 Seja $f: M^{3} \rightarrow \mathbb{Q}^{4}(c), c \leq 0$, uma imersão minima de uma variedade Riemanniana conexa com curvatura de Gauss-Kronecker constante, então $K=0$. Seja $S$ o quadrado da norma da segunda forma fundamental de $f$. Se $M^{3}$ é completa e $0<S<\infty$, então:

(i) $c=0$ e $f\left(M^{3}\right)$ se fatora em um produto Euclideano $L^{2} \times \mathbb{R}$, onde $L^{2}$ é uma superfície mínima completa de $\mathbb{R}^{3}$ com curvatura Gaussiana limitada inferiormente; ou

(ii) $c<0$ e existem uma imersão mínima $h: M^{2} \rightarrow N^{3}$, sem pontos totalmente geodésicos, de uma variedade completa orientada $M^{2}$ em uma hipersuperfície equidistante $N^{3}$ de $\mathbb{H}^{4}$ e uma isometria local $T: M^{3} \rightarrow \mathcal{N}^{1}(\tilde{h})$ tais que $f=\psi_{\tilde{h}} \circ T$.

Na afirmação acima, $\tilde{h}$ é a aplicação associada a $h$, ou seja, a aplicação $\tilde{h}:=N: M^{2} \rightarrow \mathbb{S}_{1}^{4}$, dada pela orientação $N$ de $h$, onde $\mathbb{S}_{1}^{4}$ é o espaço de De Sitter. $\mathcal{N}^{1}(\tilde{h})$ é o fibrado normal unitário tipo-tempo de $\tilde{h}$ e $\psi_{\tilde{h}}: \mathcal{N}^{1}(\tilde{h}) \rightarrow \mathbb{H}^{4}$ é a aplicação polar associada a $\tilde{h}$.

Teorema 4.2 Seja $f: M^{3} \rightarrow \mathbb{S}^{4}$ um imersão mínima de uma variedade completa, com curvatura de Gauss-Kronecker K constante. Então:

(i) $K \neq 0$ e $f\left(M^{3}\right)$ é isométrica ao toro de Clifford $S^{1}\left(\frac{\sqrt{3}}{3}\right) \times S^{2}\left(\frac{\sqrt{6}}{3}\right)$; ou

(ii) $K=0$ e se $S$, que é o quadrado da norma da segunda forma fundamental de $f$, nunca se anula e é limitada superiormente, então $f\left(M^{3}\right)$ é a imagem da aplicação polar associada à imersão super-minima $g: V \rightarrow \mathbb{S}^{4}$, com curvatura normal positiva. Além disso, se $S$ é limitada longe do zero, então $V$ é difeomorfo à esfera $S^{2}$ ou ao plano projetivo $\mathbb{R P}^{2}$ e $f\left(M^{3}\right)$ é compacto.

Observação 4.3 Seja $g: V \rightarrow \mathbb{S}^{4}$ uma imersão de uma superfície. Para $p \in V$, a elipse de curvatura $\mathcal{E}(p)$, de $g$ em $p$, é a imagem do círculo tangente pela segunda forma fundamental $h$ de $g$ em $p$ :

$$
\mathcal{E}(p)=\left\{h(X, X) \in N_{p} V \mid X \in T_{p} V,\|X\|=1\right\} .
$$

Dizemos que $g$ é super-minima se a elipse de curvatura é sempre um círculo. A curvatura normal $\mathcal{K}_{\nu}(p)$ de $g$ é definida pela equação $d \omega_{34}=-\mathcal{K}_{\nu}(p) \omega_{1} \wedge \omega_{2}$, onde $\omega_{A}, \omega_{A B}, A, B=1, \ldots, 4$, são as formas duais e de conexão, induzidas em $V$, por um referencial ortonormal $\left\{e_{1}, e_{2}, e_{3}, e_{4}\right\}$ adaptado a $g$. Além disso, seja $\mathcal{N}^{1}(g)$ o fibrado normal unitário de $g$ dado por pares $(p, \xi)$, onde $p \in V$ e $\xi$ é um vetor normal unitário de $g$ em $p$. Então a aplicação polar $\psi: \mathcal{N}^{1}(g) \rightarrow \mathbb{S}^{4}$ de $g$ é definida por $\psi(p, \xi)=\xi$. 
Para o estudo de [17], começamos com a apresentação de alguns resultados preliminares. Seja $f: M^{3} \rightarrow \mathbb{R}^{4}$ uma hipersuperfície mínima orientada, munida da métrica induzida e com um campo vetorial normal unitário $\xi$, ao longo de $f$, e denote por $\lambda_{1} \geq \lambda_{2} \geq \lambda_{3}$ suas curvaturas principais.

Assumindo que a curvatura de Gauss-Kronecker seja identicamente zero e que a segunda forma fundamental nunca se anule, as curvaturas principais satisfazem a seguinte relação

$$
\lambda_{1}=\lambda>\lambda_{2}=0>\lambda_{3}=-\lambda,
$$

onde $\lambda$ é uma função suave positiva em $M^{3}$. Desta forma, é possível escolher localmente um referencial ortonormal $\left\{e_{1}, e_{2}, e_{3}\right\}$ de direções principais, correspondentes a $\lambda, 0,-\lambda$, e nesta vizinhança podemos escrever

$$
h_{i j}=\lambda_{i} \delta_{i j} .
$$

Nessas condições, as equações de estrutura podem ser escritas da seguinte forma

$$
\begin{aligned}
d \omega_{i} & =\sum_{j=1}^{3} \omega_{i j} \wedge \omega_{j}, \quad \omega_{i j}+\omega_{j i}=0, \\
d \omega_{i j} & =\sum_{k=1}^{3} \omega_{i k} \wedge \omega_{k j}-\lambda_{i} \lambda_{j} \omega_{i} \wedge \omega_{j} .
\end{aligned}
$$

Para obter (4.2), por (1.31), (1.37) e (4.1), temos

$$
\begin{aligned}
d \omega_{i j} & =\sum_{k=1}^{3} \omega_{i k} \wedge \omega_{k j}-\frac{1}{2} \sum_{k, l=1}^{3} R_{i j k l} \omega_{k} \wedge \omega_{l} \\
& =\sum_{k=1}^{3} \omega_{i k} \wedge \omega_{k j}-\frac{1}{2} \sum_{k, l=1}^{3}\left(h_{i k} h_{j l}-h_{i l} h_{j k}\right) \omega_{k} \wedge \omega_{l} \\
& =\sum_{k=1}^{3} \omega_{i k} \wedge \omega_{k j}-\frac{1}{2} \sum_{k, l=1}^{3} \lambda_{i} \lambda_{j}\left(\delta_{i k} \delta_{j l}-\delta_{i l} \delta_{j k}\right) \omega_{k} \wedge \omega_{l} \\
& =\sum_{k=1}^{3} \omega_{i k} \wedge \omega_{k j}-\lambda_{i} \lambda_{j} \omega_{i} \wedge \omega_{j} .
\end{aligned}
$$

Duas funções terão papel crucial na prova do resultado e as definiremos agora

$$
\begin{aligned}
u & :=\omega_{12}\left(e_{3}\right), \\
v & :=e_{2}(\ln \lambda) .
\end{aligned}
$$

Lema 4.4 Seja $f: M^{3} \rightarrow \mathbb{R}^{4}$ uma hipersuperfície minima orientada com curvatura de GaussKronecker identicamente zero e com segunda forma fundamental nunca nula. Seja $\left\{e_{1}, e_{2}, e_{3}\right\}$ um referencial ortonormal local de direçôes principais correspondentes a $\lambda_{1}=\lambda, \lambda_{2}=0, \lambda_{3}=-\lambda$, onde $\lambda$ é uma função suave positiva em $M^{3}$. Então, para as formas de conexão, valem

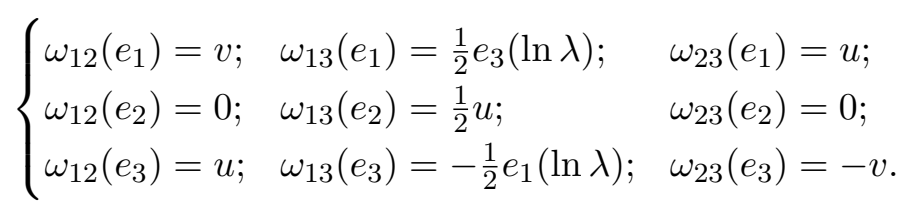

Os colchetes entre dois elementos do referencial ortonormal são dados por 


$$
\left\{\begin{array}{l}
{\left[e_{1}, e_{2}\right]=-v e_{1}+\frac{1}{2} u e_{3}} \\
{\left[e_{1}, e_{3}\right]=-\frac{1}{2} e_{3}(\ln \lambda) e_{1}-2 u e_{2}+\frac{1}{2} e_{1}(\ln \lambda) e_{3}} \\
{\left[e_{2}, e_{3}\right]=\frac{1}{2} u e_{1}+v e_{3}}
\end{array}\right.
$$

As derivadas direcionais das funções $u$ e $v$ são dadas por

$$
\begin{cases}e_{1}(u)=e_{3}(v) ; & e_{2}(u)=2 u v ; \\ e_{1}(v)=-e_{3}(u) ; & e_{2}(v)=v^{2}-u^{2} .\end{cases}
$$

Além disso, temos

$$
e_{1} e_{1}(\ln \lambda)+e_{3} e_{3}(\ln \lambda)-\frac{1}{2}\left(e_{1}(\ln \lambda)\right)^{2}-\frac{1}{2}\left(e_{3}(\ln \lambda)\right)^{2}-2 v^{2}-4 u^{2}+2 \lambda^{2}=0 .
$$

Demonstração: A derivada covariante $\nabla h$ da segunda forma fundamental $h$ de $M^{3}$ com componentes $h_{i j ; k}$ é dada por

$$
\sum_{k=1}^{3} h_{i j ; k} \omega_{k}=d h_{i j}+\sum_{k=1}^{3} h_{k j} \omega_{k i}+\sum_{k=1}^{3} h_{i k} \omega_{k j} .
$$

Como $h_{i j}=\lambda_{i} \delta_{i j}$, podemos escrever

$$
\sum_{k=1}^{3} h_{i j ; k} \omega_{k}=\delta_{i j} d \lambda_{i}+\sum_{k=1}^{3} \lambda_{j} \delta_{j k} \omega_{k i}+\sum_{k=1}^{3} \lambda_{i} \delta_{i k} \omega_{k j} .
$$

Logo, para quaisquer $i, j, k \in\{1,2,3\}$, vale

$$
\begin{aligned}
h_{i j ; k} & =\delta_{i j} d \lambda_{i}\left(e_{k}\right)+\lambda_{j} \omega_{j i}\left(e_{k}\right)+\lambda_{i} \omega_{i j}\left(e_{k}\right) \\
& =\delta_{i j} e_{k}\left(\lambda_{i}\right)+\left(\lambda_{i}-\lambda_{j}\right) \omega_{i j}\left(e_{k}\right) .
\end{aligned}
$$

Em particular, temos

$$
h_{j j ; i}=\delta_{j j} e_{i}\left(\lambda_{j}\right)+\left(\lambda_{j}-\lambda_{j}\right) \omega_{j j}\left(e_{i}\right),
$$

ou seja,

$$
e_{i}\left(\lambda_{j}\right)=h_{j j ; i}
$$

Por (4.7), temos

$$
h_{i j ; j}=\delta_{i j} e_{j}\left(\lambda_{i}\right)+\left(\lambda_{i}-\lambda_{j}\right) \omega_{i j}\left(e_{j}\right) .
$$

Utilizando as equações de Codazzi e (4.8) chegamos a

$$
e_{i}\left(\lambda_{j}\right)=h_{j j ; i}=h_{i j ; j}=\delta_{i j} e_{j}\left(\lambda_{i}\right)+\left(\lambda_{i}-\lambda_{j}\right) \omega_{i j}\left(e_{j}\right)
$$

ou seja,

$$
e_{i}\left(\lambda_{j}\right)=\left(\lambda_{i}-\lambda_{j}\right) \omega_{i j}\left(e_{j}\right)=\left(\lambda_{j}-\lambda_{i}\right) \omega_{j i}\left(e_{j}\right), \quad i \neq j .
$$

Portanto, renomeando os índices, obtemos

$$
\omega_{i j}\left(e_{i}\right)=\frac{1}{\lambda_{i}-\lambda_{j}} e_{j}\left(\lambda_{i}\right), \quad i \neq j .
$$

Utilizando (4.7), temos

$$
\left\{\begin{array}{l}
h_{12 ; 3}=\delta_{12} e_{3}\left(\lambda_{1}\right)+\left(\lambda_{1}-\lambda_{2}\right) \omega_{12}\left(e_{3}\right)=\lambda \omega_{12}\left(e_{3}\right) \\
h_{23 ; 1}=\delta_{23} e_{1}\left(\lambda_{2}\right)+\left(\lambda_{2}-\lambda_{3}\right) \omega_{23}\left(e_{1}\right)=\lambda \omega_{23}\left(e_{1}\right) \\
h_{13 ; 2}=\delta_{13} e_{2}\left(\lambda_{1}\right)+\left(\lambda_{1}-\lambda_{3}\right) \omega_{13}\left(e_{2}\right)=2 \lambda \omega_{13}\left(e_{2}\right) .
\end{array}\right.
$$


Pelas equações de Codazzi, obtemos

$$
u=\omega_{12}\left(e_{3}\right)=\omega_{23}\left(e_{1}\right)=2 \omega_{13}\left(e_{2}\right),
$$

o que demonstra 3 das 9 equações de (4.3). Para as outras 6, utilizando (4.9), obtemos

$$
\begin{aligned}
& \omega_{12}\left(e_{1}\right)=\frac{1}{\lambda_{1}-\lambda_{2}} e_{2}\left(\lambda_{1}\right)=\frac{1}{\lambda} e_{2}(\lambda)=e_{2}(\ln \lambda)=v, \\
& \omega_{13}\left(e_{1}\right)=\frac{1}{\lambda_{1}-\lambda_{3}} e_{3}\left(\lambda_{1}\right)=\frac{1}{2 \lambda} e_{3}(\lambda)=\frac{1}{2} e_{3}(\ln \lambda), \\
& \omega_{12}\left(e_{2}\right)=-\omega_{21}\left(e_{2}\right)=-\frac{1}{\lambda_{2}-\lambda_{1}} e_{1}\left(\lambda_{2}\right)=\frac{1}{\lambda} e_{1}(0)=0, \\
& \omega_{23}\left(e_{2}\right)=\frac{1}{\lambda_{2}-\lambda_{3}} e_{3}\left(\lambda_{2}\right)=\frac{1}{\lambda} e_{3}(0)=0, \\
& \omega_{13}\left(e_{3}\right)=-\omega_{31}\left(e_{3}\right)=-\frac{1}{\lambda_{3}-\lambda_{1}} e_{1}\left(\lambda_{3}\right)=-\frac{1}{2 \lambda} e_{1}(\lambda)=-\frac{1}{2} e_{1}(\ln \lambda), \\
& \omega_{23}\left(e_{3}\right)=-\omega_{32}\left(e_{3}\right)=-\frac{1}{\lambda_{3}-\lambda_{2}} e_{2}\left(\lambda_{3}\right)=-\frac{1}{\lambda} e_{2}(\lambda)=-e_{2}(\ln \lambda)=-v .
\end{aligned}
$$

Pela simetria da conexão e por (1.11), temos

$$
\begin{aligned}
{\left[e_{i}, e_{j}\right] } & =\nabla_{e_{i}} e_{j}-\nabla_{e_{j}} e_{i} \\
& =\sum_{k=1}^{3} \omega_{j k}\left(e_{i}\right) e_{k}-\sum_{k=1}^{3} \omega_{i k}\left(e_{j}\right) e_{k}=\sum_{k=1}^{3}\left(\omega_{j k}\left(e_{i}\right)-\omega_{i k}\left(e_{j}\right)\right) e_{k} \\
& =\left(\omega_{j 1}\left(e_{i}\right)-\omega_{i 1}\left(e_{j}\right)\right) e_{1}+\left(\omega_{j 2}\left(e_{i}\right)-\omega_{i 2}\left(e_{j}\right)\right) e_{2}+\left(\omega_{j 3}\left(e_{i}\right)-\omega_{i 3}\left(e_{j}\right)\right) e_{3} .
\end{aligned}
$$

Portanto, valem

$$
\begin{aligned}
{\left[e_{1}, e_{2}\right] } & =\left(\omega_{21}\left(e_{1}\right)-\omega_{11}\left(e_{2}\right)\right) e_{1}+\left(\omega_{22}\left(e_{1}\right)-\omega_{12}\left(e_{2}\right)\right) e_{2}+\left(\omega_{23}\left(e_{1}\right)-\omega_{13}\left(e_{2}\right)\right) e_{3} \\
& =(-v-0) e_{1}+(0-0) e_{2}+\left(u-\frac{1}{2} u\right) e_{3} \\
& =-v e_{1}+\frac{1}{2} u e_{3} \\
{\left[e_{1}, e_{3}\right] } & =\left(\omega_{31}\left(e_{1}\right)-\omega_{11}\left(e_{3}\right)\right) e_{1}+\left(\omega_{32}\left(e_{1}\right)-\omega_{12}\left(e_{3}\right)\right) e_{2}+\left(\omega_{33}\left(e_{1}\right)-\omega_{13}\left(e_{3}\right)\right) e_{3} \\
& =\left(-\frac{1}{2} e_{3}(\ln \lambda)-0\right) e_{1}+(-u-u) e_{2}+\left(0+\frac{1}{2} e_{1}(\ln \lambda)\right) e_{3} \\
& =-\frac{1}{2} e_{3}(\ln \lambda) e_{1}-2 u e_{2}+\frac{1}{2} e_{1}(\ln \lambda) e_{3}, \\
{\left[e_{2}, e_{3}\right] } & =\left(\omega_{31}\left(e_{2}\right)-\omega_{21}\left(e_{3}\right)\right) e_{1}+\left(\omega_{32}\left(e_{2}\right)-\omega_{22}\left(e_{3}\right)\right) e_{2}+\left(\omega_{33}\left(e_{2}\right)-\omega_{23}\left(e_{3}\right)\right) e_{3} \\
& =\left(-\frac{1}{2} u+u\right) e_{1}+(0-0) e_{2}+(0+v) e_{3} \\
& =\frac{1}{2} u e_{1}+v e_{3},
\end{aligned}
$$

o que demonstra (4.4).

Por (4.2), temos

$$
\begin{aligned}
d \omega_{k 2}\left(e_{i}, e_{j}\right) & =\sum_{l=1}^{3} \omega_{k l} \wedge \omega_{l 2}\left(e_{i}, e_{j}\right)-\lambda_{k} \lambda_{2} \omega_{1} \wedge \omega_{2}\left(e_{i}, e_{j}\right) \\
& =\sum_{l=1}^{3} \omega_{k l} \wedge \omega_{l 2}\left(e_{i}, e_{j}\right) .
\end{aligned}
$$

Pela propriedade (e) da Proposição 1.3.3, vale

$$
d \omega_{k 2}\left(e_{i}, e_{j}\right)=e_{i}\left(\omega_{k 2}\left(e_{j}\right)\right)-e_{j}\left(\omega_{k 2}\left(e_{i}\right)\right)-\omega_{k 2}\left(\left[e_{i}, e_{j}\right]\right),
$$

ou seja, 


$$
\begin{aligned}
e_{i}\left(\omega_{k 2}\left(e_{j}\right)\right) & =d \omega_{k 2}\left(e_{i}, e_{j}\right)+e_{j}\left(\omega_{k 2}\left(e_{i}\right)\right)+\omega_{k 2}\left(\left[e_{i}, e_{j}\right]\right) \\
& =\sum_{l=1}^{3} \omega_{k l} \wedge \omega_{l 2}\left(e_{i}, e_{j}\right)+e_{j}\left(\omega_{k 2}\left(e_{i}\right)\right)+\omega_{k 2}\left(\left[e_{i}, e_{j}\right]\right) .
\end{aligned}
$$

Para $k=1$, temos

$$
\begin{aligned}
e_{i}\left(\omega_{12}\left(e_{j}\right)\right) & =\sum_{l=1}^{3} \omega_{1 l} \wedge \omega_{l 2}\left(e_{i}, e_{j}\right)+e_{j}\left(\omega_{12}\left(e_{i}\right)\right)+\omega_{12}\left(\left[e_{i}, e_{j}\right]\right) \\
& =\omega_{13} \wedge \omega_{32}\left(e_{i}, e_{j}\right)+e_{j}\left(\omega_{12}\left(e_{i}\right)\right)+\omega_{12}\left(\left[e_{i}, e_{j}\right]\right) \\
& =\omega_{23}\left(e_{i}\right) \omega_{13}\left(e_{j}\right)-\omega_{23}\left(e_{j}\right) \omega_{13}\left(e_{i}\right)+e_{j}\left(\omega_{12}\left(e_{i}\right)\right)+\omega_{12}\left(\left[e_{i}, e_{j}\right]\right) .
\end{aligned}
$$

Para $k=3$, temos

$$
\begin{aligned}
e_{i}\left(\omega_{32}\left(e_{j}\right)\right) & =\sum_{l=1}^{3} \omega_{3 l} \wedge \omega_{l 2}\left(e_{i}, e_{j}\right)+e_{j}\left(\omega_{32}\left(e_{i}\right)\right)+\omega_{32}\left(\left[e_{i}, e_{j}\right]\right) \\
& =\omega_{31} \wedge \omega_{12}\left(e_{i}, e_{j}\right)+e_{j}\left(\omega_{32}\left(e_{i}\right)\right)+\omega_{32}\left(\left[e_{i}, e_{j}\right]\right) \\
& =\omega_{12} \wedge \omega_{13}\left(e_{i}, e_{j}\right)-e_{j}\left(\omega_{23}\left(e_{i}\right)\right)-\omega_{23}\left(\left[e_{i}, e_{j}\right]\right) \\
& =\omega_{12}\left(e_{i}\right) \omega_{13}\left(e_{j}\right)-\omega_{12}\left(e_{j}\right) \omega_{13}\left(e_{i}\right)-e_{j}\left(\omega_{23}\left(e_{i}\right)\right)-\omega_{23}\left(\left[e_{i}, e_{j}\right]\right) .
\end{aligned}
$$

Por (4.10) e (4.11), temos

$$
\begin{aligned}
e_{1}(u) & =e_{1}\left(\omega_{12}\left(e_{3}\right)\right) \\
& =\omega_{23}\left(e_{1}\right) \omega_{13}\left(e_{3}\right)-\omega_{23}\left(e_{3}\right) \omega_{13}\left(e_{1}\right)+e_{3}\left(\omega_{12}\left(e_{1}\right)\right)+\omega_{12}\left(\left[e_{1}, e_{3}\right]\right) \\
& =-\frac{1}{2} u e_{1}(\ln \lambda)+\frac{1}{2} v e_{3}(\ln \lambda)+e_{3}(v)+\omega_{12}\left(-\frac{1}{2} e_{3}(\ln \lambda) e_{1}-2 u e_{2}+\frac{1}{2} e_{1}(\ln \lambda) e_{3}\right) \\
& =-\frac{1}{2} u e_{1}(\ln \lambda)+\frac{1}{2} v e_{3}(\ln \lambda)+e_{3}(v)-\frac{1}{2} e_{3}(\ln \lambda) \omega_{12}\left(e_{1}\right)-2 u \omega_{12}\left(e_{2}\right)+\frac{1}{2} e_{1}(\ln \lambda) \omega_{12}\left(e_{3}\right) \\
& =-\frac{1}{2} u e_{1}(\ln \lambda)+\frac{1}{2} v e_{3}(\ln \lambda)+e_{3}(v)-\frac{1}{2} v e_{3}(\ln \lambda)-0+\frac{1}{2} u e_{1}(\ln \lambda) \\
& =e_{3}(v), \\
e_{1}(v) & =e_{1}\left(\omega_{32}\left(e_{3}\right)\right) \\
& =\omega_{12}\left(e_{1}\right) \omega_{13}\left(e_{3}\right)-\omega_{12}\left(e_{3}\right) \omega_{13}\left(e_{1}\right)-e_{3}\left(\omega_{23}\left(e_{1}\right)\right)-\omega_{23}\left(\left[e_{1}, e_{3}\right]\right) \\
& =-\frac{1}{2} v e_{1}(\ln \lambda)-\frac{1}{2} u e_{3}(\ln \lambda)-e_{3}(u)-\omega_{23}\left(-\frac{1}{2} e_{3}(\ln \lambda) e_{1}-2 u e_{2}+\frac{1}{2} e_{1}(\ln \lambda) e_{3}\right) \\
& =-\frac{1}{2} v e_{1}(\ln \lambda)-\frac{1}{2} u e_{3}(\ln \lambda)-e_{3}(u)+\frac{1}{2} e_{3}(\ln \lambda) \omega_{23}\left(e_{1}\right)+2 u \omega_{23}\left(e_{2}\right)-\frac{1}{2} e_{1}(\ln \lambda) \omega_{23}\left(e_{3}\right) \\
& =-\frac{1}{2} v e_{1}(\ln \lambda)-\frac{1}{2} u e_{3}(\ln \lambda)-e_{3}(u)+\frac{1}{2} u e_{3}(\ln \lambda)+0+\frac{1}{2} v e_{1}(\ln \lambda) \\
& =-e_{3}(u), \\
e_{2}(u) & =e_{2}\left(\omega_{12}\left(e_{3}\right)\right) \\
& =\omega_{23}\left(e_{2}\right) \omega_{13}\left(e_{3}\right)-\omega_{23}\left(e_{3}\right) \omega_{13}\left(e_{2}\right)+e_{3}\left(\omega_{12}\left(e_{2}\right)\right)+\omega_{12}\left(\left[e_{2}, e_{3}\right]\right) \\
& =0+\frac{1}{2} u v+0+\omega_{12}\left(\frac{1}{2} u e_{1}+v e_{3}\right) \\
& =\frac{1}{2} u v+\frac{1}{2} u \omega_{12}\left(e_{1}\right)+v \omega_{12}\left(e_{3}\right) \\
& =\frac{1}{2} u v+\frac{1}{2} u v+u v \\
& =2 u v \\
e_{2}(v) & =e_{2}\left(\omega_{12}\left(e_{1}\right)\right) \\
& =\omega_{23}\left(e_{2}\right) \omega_{13}\left(e_{1}\right)-\omega_{23}\left(e_{1}\right) \omega_{13}\left(e_{2}\right)+e_{1}\left(\omega_{12}\left(e_{2}\right)\right)+\omega_{12}\left(\left[e_{2}, e_{1}\right]\right) \\
& =0-\frac{1}{2} u^{2}+0-\omega_{12}\left(-v e_{1}+\frac{1}{2} u e_{3}\right) \\
& =-\frac{1}{2} u^{2}+v \omega_{12}\left(e_{1}\right)-\frac{1}{2} u \omega_{12}\left(e_{3}\right) \\
& =-\frac{1}{2} u^{2}+v^{2}-\frac{1}{2} u^{2} \\
& =v^{2}-u^{2}, \\
&
\end{aligned}
$$


o que demonstra (4.5).

Pela propriedade (e) da Proposição 1.3.3, vale

$$
d \omega_{13}\left(e_{1}, e_{3}\right)=e_{1}\left(\omega_{13}\left(e_{3}\right)\right)-e_{3}\left(\omega_{13}\left(e_{1}\right)\right)-\omega_{13}\left(\left[e_{1}, e_{3}\right]\right) .
$$

Logo,

$$
\begin{aligned}
0= & -2 e_{1}\left(\omega_{13}\left(e_{3}\right)\right)+2 e_{3}\left(\omega_{13}\left(e_{1}\right)\right)+2 \omega_{13}\left(\left[e_{1}, e_{3}\right]\right)+2 d \omega_{13}\left(e_{1}, e_{3}\right) \\
= & -2 e_{1}\left(-\frac{1}{2} e_{1}(\ln \lambda)\right)+2 e_{3}\left(\frac{1}{2} e_{3}(\ln \lambda)\right)+2 \omega_{13}\left(-\frac{1}{2} e_{3}(\ln \lambda) e_{1}-2 u e_{2}+\frac{1}{2} e_{1}(\ln \lambda) e_{3}\right) \\
& +2\left(\sum_{k=1}^{3} \omega_{1 k} \wedge \omega_{k 3}\left(e_{1}, e_{3}\right)-\lambda_{1} \lambda_{3} \omega_{1} \wedge \omega_{3}\left(e_{1}, e_{3}\right)\right) \\
= & e_{1} e_{1}(\ln \lambda)+e_{3} e_{3}(\ln \lambda)-e_{3}(\ln \lambda) \omega_{13}\left(e_{1}\right)-4 u \omega_{13}\left(e_{2}\right)+e_{1}(\ln \lambda) \omega_{13}\left(e_{3}\right) \\
& +2\left(\omega_{12} \wedge \omega_{23}\left(e_{1}, e_{3}\right)+\lambda^{2}\right) \\
= & e_{1} e_{1}(\ln \lambda)+e_{3} e_{3}(\ln \lambda)-\frac{1}{2}\left(e_{3}(\ln \lambda)\right)^{2}-2 u^{2}-\frac{1}{2}\left(e_{1}(\ln \lambda)\right)^{2} \\
& +2\left(\omega_{12}\left(e_{1}\right) \omega_{23}\left(e_{3}\right)-\omega_{12}\left(e_{3}\right) \omega_{23}\left(e_{1}\right)\right)+2 \lambda^{2} \\
= & e_{1} e_{1}(\ln \lambda)+e_{3} e_{3}(\ln \lambda)-\frac{1}{2}\left(e_{3}(\ln \lambda)\right)^{2}-2 u^{2}-\frac{1}{2}\left(e_{1}(\ln \lambda)\right)^{2}+2\left(-v^{2}-u^{2}\right)+2 \lambda^{2} \\
= & e_{1} e_{1}(\ln \lambda)+e_{3} e_{3}(\ln \lambda)-\frac{1}{2}\left(e_{1}(\ln \lambda)\right)^{2}-\frac{1}{2}\left(e_{3}(\ln \lambda)\right)^{2}-2 v^{2}-4 u^{2}+2 \lambda^{2},
\end{aligned}
$$

o que demonstra (4.6).

Precisaremos de um resultado de Cheng e Yau [5] que será enunciado no seguinte lema, cuja demonstração segue ideias apresentadas por Nishikawa [22].

Lema 4.5 Seja $M^{n}$ uma variedade Riemanniana completa $n$-dimensional, $n \geq 2$, com curvatura de Ricci Ric $\geq-(n-1) k^{2}$, onde $k$ é uma constante positiva. Suponha que g é uma função suave não-negativa em $M^{n}$ satisfazendo

$$
\Delta g \geq c g^{2},
$$

onde c é uma constante positiva. Então g é identicamente nula.

Demonstração: Tome um ponto $x_{0} \in M^{n}$. Se $g$ atinge seu máximo em $x_{0}$, então por (4.12) temos

$$
0 \geq \Delta g\left(x_{0}\right) \geq c g^{2}\left(x_{0}\right)
$$

Mas c é uma constante positiva, então

$$
g^{2}\left(x_{0}\right) \leq 0
$$

Como g é uma função não-negativa, concluímos que $g\left(x_{0}\right)=0$ e o resultado é verdadeiro. Provaremos que $g(x) \leq g\left(x_{0}\right)$, para qualquer ponto $x \in M^{n}$. Suponha, por absurdo, que exista um ponto $x_{1}$ tal que $g\left(x_{0}\right)<g\left(x_{1}\right)$. Definimos $\beta=d\left(x_{0}, x_{1}\right)$ e denotamos por $B_{a}\left(x_{0}\right)$ a bola geodésica de raio $a$ centrada em $x_{0}$, onde $d$ é a distância em $M^{n}$. Consideremos a função $G: B_{a}\left(x_{0}\right) \rightarrow \mathbb{R}$ dada por

$$
G(x)=\left(a^{2}-\rho^{2}(x)\right)^{2} g(x),
$$

onde $a>\beta$ e $\rho(x)=d\left(x, x_{0}\right)$. Como $\left.G\right|_{\partial B_{a}\left(x_{0}\right)}=0$ e $G$ é não-negativa, deduzimos que $G$ atinge seu máximo em algum ponto $x_{2} \in B_{a}\left(x_{0}\right)$, com $g\left(x_{2}\right)>0$. Primeiro assumimos que $x_{2}$ não é um ponto de corte de $x_{0}$. Então $G$ é suave em uma vizinhança de $x_{2}$ e pelo princípio do máximo temos

$$
\operatorname{grad} G\left(x_{2}\right)=0, \quad \Delta G\left(x_{2}\right) \leq 0 .
$$

Como $\rho(x)$ é uma função distância temos que $\|\operatorname{grad} \rho(x)\|^{2}=1$. Com isso, 


$$
\operatorname{grad}\left(a^{2}-\rho^{2}(x)\right)=-\operatorname{grad} \rho^{2}(x)=-2 \rho(x) \operatorname{grad} \rho(x),
$$

$$
\begin{gathered}
\operatorname{grad}\left(\left(a^{2}-\rho^{2}(x)\right)^{2}\right)=2\left(a^{2}-\rho^{2}(x)\right) \operatorname{grad}\left(a^{2}-\rho^{2}(x)\right)=-4 \rho(x)\left(a^{2}-\rho^{2}(x)\right) \operatorname{grad} \rho(x), \\
\Delta\left(a^{2}-\rho^{2}(x)\right)=-\Delta \rho^{2}(x)=-2 \rho(x) \Delta \rho(x)-2\|\operatorname{grad} \rho(x)\|^{2}=-2(1+\rho(x) \Delta \rho(x))
\end{gathered}
$$

e

$$
\begin{aligned}
\Delta\left(\left(a^{2}-\rho^{2}(x)\right)^{2}\right) & =2\left(a^{2}-\rho^{2}(x)\right) \Delta\left(a^{2}-\rho^{2}(x)\right)+2\left\|\operatorname{grad}\left(a^{2}-\rho^{2}(x)\right)\right\|^{2} \\
& =-4\left(a^{2}-\rho^{2}(x)\right)(1+\rho(x) \Delta \rho(x))+8 \rho^{2}(x) .
\end{aligned}
$$

Logo,

$$
\begin{aligned}
\operatorname{grad} G(x) & =\operatorname{grad}\left(\left(a^{2}-\rho^{2}(x)\right)^{2} g(x)\right) \\
& =\left(a^{2}-\rho^{2}(x)\right)^{2} \operatorname{grad} g(x)+g(x) \operatorname{grad}\left(\left(a^{2}-\rho^{2}(x)\right)^{2}\right) \\
& =\left(a^{2}-\rho^{2}(x)\right)^{2} \operatorname{grad} g(x)-4 g(x) \rho(x)\left(a^{2}-\rho^{2}(x)\right) \operatorname{grad} \rho(x)
\end{aligned}
$$

e

$$
\begin{aligned}
\Delta G(x)= & \Delta\left(\left(a^{2}-\rho^{2}(x)\right)^{2} g(x)\right) \\
= & \left(a^{2}-\rho^{2}(x)\right)^{2} \Delta g(x)+g(x) \Delta\left(\left(a^{2}-\rho^{2}(x)\right)^{2}\right) \\
& +2\left\langle\operatorname{grad}\left(\left(a^{2}-\rho^{2}(x)\right)^{2}\right), \operatorname{grad} g(x)\right\rangle \\
= & \left(a^{2}-\rho^{2}(x)\right)^{2} \Delta g(x)+g(x)\left(-4\left(a^{2}-\rho^{2}(x)\right)(1+\rho(x) \Delta \rho(x))+8 \rho^{2}(x)\right) \\
& +2\left\langle-4 \rho(x)\left(a^{2}-\rho^{2}(x)\right) \operatorname{grad} \rho(x), \operatorname{grad} g(x)\right\rangle \\
= & \left(a^{2}-\rho^{2}(x)\right)^{2} \Delta g(x)-4 g(x)\left(a^{2}-\rho^{2}(x)\right)(1+\rho(x) \Delta \rho(x))+8 g(x) \rho^{2}(x) \\
& -8 \rho(x)\left(a^{2}-\rho^{2}(x)\right)\langle\operatorname{grad} \rho(x), \operatorname{grad} g(x)\rangle .
\end{aligned}
$$

Por (4.13), temos

$$
0=\operatorname{grad} G\left(x_{2}\right)=\left(a^{2}-\rho^{2}\left(x_{2}\right)\right)^{2} \operatorname{grad} g\left(x_{2}\right)-4 g\left(x_{2}\right) \rho\left(x_{2}\right)\left(a^{2}-\rho^{2}\left(x_{2}\right)\right) \operatorname{grad} \rho\left(x_{2}\right),
$$

ou seja,

$$
\frac{\operatorname{grad} g\left(x_{2}\right)}{g\left(x_{2}\right)}=\frac{4 \rho\left(x_{2}\right) \operatorname{grad} \rho\left(x_{2}\right)}{a^{2}-\rho^{2}\left(x_{2}\right)} .
$$

Vale também

$$
\begin{aligned}
0 \geq & \Delta G\left(x_{2}\right) \\
= & \left(a^{2}-\rho^{2}\left(x_{2}\right)\right)^{2} \Delta g\left(x_{2}\right)-4 g\left(x_{2}\right)\left(a^{2}-\rho^{2}\left(x_{2}\right)\right)\left(1+\rho\left(x_{2}\right) \Delta \rho\left(x_{2}\right)\right) \\
& +8 g\left(x_{2}\right) \rho^{2}\left(x_{2}\right)-8 \rho\left(x_{2}\right)\left(a^{2}-\rho^{2}\left(x_{2}\right)\right)\left\langle\operatorname{grad} \rho\left(x_{2}\right), \operatorname{grad} g\left(x_{2}\right)\right\rangle
\end{aligned}
$$




$$
\begin{aligned}
= & \left(a^{2}-\rho^{2}\left(x_{2}\right)\right)^{2} \Delta g\left(x_{2}\right)-4 g\left(x_{2}\right)\left(a^{2}-\rho^{2}\left(x_{2}\right)\right)\left(1+\rho\left(x_{2}\right) \Delta \rho\left(x_{2}\right)\right) \\
& +8 g\left(x_{2}\right) \rho^{2}\left(x_{2}\right)-8 \rho\left(x_{2}\right)\left(a^{2}-\rho^{2}\left(x_{2}\right)\right)\left\langle\operatorname{grad} \rho\left(x_{2}\right), \frac{4 g\left(x_{2}\right) \rho\left(x_{2}\right) \operatorname{grad} \rho\left(x_{2}\right)}{a^{2}-\rho^{2}\left(x_{2}\right)}\right\rangle \\
= & \left(a^{2}-\rho^{2}\left(x_{2}\right)\right)^{2} \Delta g\left(x_{2}\right)-4 g\left(x_{2}\right)\left(a^{2}-\rho^{2}\left(x_{2}\right)\right)\left(1+\rho\left(x_{2}\right) \Delta \rho\left(x_{2}\right)\right) \\
& +8 g\left(x_{2}\right) \rho^{2}\left(x_{2}\right)-32 g\left(x_{2}\right) \rho^{2}\left(x_{2}\right) \\
= & \left(a^{2}-\rho^{2}\left(x_{2}\right)\right)^{2} \Delta g\left(x_{2}\right)-4 g\left(x_{2}\right)\left(a^{2}-\rho^{2}\left(x_{2}\right)\right)\left(1+\rho\left(x_{2}\right) \Delta \rho\left(x_{2}\right)\right)-24 g\left(x_{2}\right) \rho^{2}\left(x_{2}\right),
\end{aligned}
$$

ou seja,

$$
\frac{\Delta g\left(x_{2}\right)}{g\left(x_{2}\right)} \leq \frac{24 \rho^{2}\left(x_{2}\right)}{\left(a^{2}-\rho^{2}\left(x_{2}\right)\right)^{2}}+\frac{4\left(1+\rho\left(x_{2}\right) \Delta \rho\left(x_{2}\right)\right)}{a^{2}-\rho^{2}\left(x_{2}\right)} .
$$

Seja $\sigma^{\prime}(t)$ o vetor tangente da única geodésica mínima $\sigma$ ligando $x_{0}$ a $x_{2}$ e $\operatorname{Ric}\left(\sigma^{\prime}(t)\right)$ a curvatura de Ricci de $M^{n}$ na direção $\sigma^{\prime}(t)$, então, como Ric $\geq-(n-1) k^{2}$, pelo Lema 1 de [32] vale a seguinte desigualdade

$$
\begin{aligned}
\Delta \rho\left(x_{2}\right) & \leq \min _{0 \leq \alpha \leq \rho\left(x_{2}\right)}\left[\frac{n-1}{\rho\left(x_{2}\right)-\alpha}-\frac{1}{\left(\rho\left(x_{2}\right)-\alpha\right)^{2}} \int_{\alpha}^{\rho\left(x_{2}\right)}(t-\alpha)^{2} \operatorname{Ric}\left(\sigma^{\prime}(t)\right) d t\right] \\
& \leq \min _{0 \leq \alpha \leq \rho\left(x_{2}\right)}\left[\frac{n-1}{\rho\left(x_{2}\right)-\alpha}+\frac{(n-1) k^{2}}{\left(\rho\left(x_{2}\right)-\alpha\right)^{2}} \int_{\alpha}^{\rho\left(x_{2}\right)}(t-\alpha)^{2} d t\right] \\
& =(n-1) \min _{0 \leq \alpha \leq \rho\left(x_{2}\right)}\left[\frac{1}{\rho\left(x_{2}\right)-\alpha}+\left.\frac{k^{2}}{\left(\rho\left(x_{2}\right)-\alpha\right)^{2}} \frac{(t-\alpha)^{3}}{3}\right|_{\alpha} ^{\rho\left(x_{2}\right)}\right] \\
& =(n-1) \min _{0 \leq \alpha \leq \rho\left(x_{2}\right)}\left[\frac{1}{\rho\left(x_{2}\right)-\alpha}+\frac{k^{2}\left(\rho\left(x_{2}\right)-\alpha\right)}{3}\right] \\
& =(n-1) \min _{0 \leq y \leq \rho\left(x_{2}\right)}\left[\frac{1}{y}+\frac{k^{2} y}{3}\right] \\
& \leq(n-1) \min _{0 \leq y \leq \rho\left(x_{2}\right)}\left[\frac{1}{y}+k^{2} y\right] .
\end{aligned}
$$

O mínimo de $\frac{1}{y}+k^{2} y$, com $y \in \mathbb{R}^{+}$, ocorre em $\frac{1}{k}$. Como estamos com um intervalo reduzido, $\left[0, \rho\left(x_{2}\right)\right]$, devemos analisar as possibilidades de $\frac{1}{k}$ estar ou não contido no intervalo. Se $\frac{1}{k}<\rho\left(x_{2}\right)$, o mínimo ocorre em $\frac{1}{k}$ e temos

$$
\begin{aligned}
\Delta \rho\left(x_{2}\right) & \leq(n-1) \min _{0 \leq y \leq \rho\left(x_{2}\right)}\left[\frac{1}{y}+k^{2} y\right] \\
& =(n-1)(k+k) \\
& \leq(n-1)\left(\frac{1}{\rho\left(x_{2}\right)}+2 k\right) .
\end{aligned}
$$

Se $\frac{1}{k} \geq \rho\left(x_{2}\right)$, ou seja, $k \leq \frac{1}{\rho\left(x_{2}\right)}$, o mínimo ocorre em $\rho\left(x_{2}\right)$ e temos

$$
\begin{aligned}
\Delta \rho\left(x_{2}\right) & \leq(n-1) \min _{0 \leq y \leq \rho\left(x_{2}\right)}\left[\frac{1}{y}+k^{2} y\right] \\
& =(n-1)\left(\frac{1}{\rho\left(x_{2}\right)}+k^{2} \rho\left(x_{2}\right)\right) \\
& \leq(n-1)\left(\frac{1}{\rho\left(x_{2}\right)}+k \frac{1}{\rho\left(x_{2}\right)} \rho\left(x_{2}\right)\right)
\end{aligned}
$$




$$
\begin{aligned}
& =(n-1)\left(\frac{1}{\rho\left(x_{2}\right)}+k\right) \\
& \leq(n-1)\left(\frac{1}{\rho\left(x_{2}\right)}+2 k\right) .
\end{aligned}
$$

Logo,

$$
\rho\left(x_{2}\right) \Delta \rho\left(x_{2}\right) \leq(n-1)\left(1+2 k \rho\left(x_{2}\right)\right) .
$$

Por (4.15), obtemos

$$
\begin{aligned}
1+\rho\left(x_{2}\right) \Delta \rho\left(x_{2}\right) & \leq 1+(n-1)\left(1+2 k \rho\left(x_{2}\right)\right) \\
& =n+2(n-1) k \rho\left(x_{2}\right) \\
& \leq 2 n\left(1+k \rho\left(x_{2}\right)\right)
\end{aligned}
$$

Desta forma, por (4.12), (4.14) e (4.16), temos

$$
\begin{aligned}
G\left(x_{2}\right) & =\left(a^{2}-\rho^{2}(x)\right)^{2} g\left(x_{2}\right) \\
& \leq \frac{\left(a^{2}-\rho^{2}(x)\right)^{2} \Delta g\left(x_{2}\right)}{c g\left(x_{2}\right)} \\
& \leq \frac{1}{c}\left(24 \rho^{2}\left(x_{2}\right)+4\left(1+\rho\left(x_{2}\right) \Delta \rho\left(x_{2}\right)\right)\left(a^{2}-\rho^{2}\left(x_{2}\right)\right)\right) \\
& \leq \frac{1}{c}\left(24 a^{2}+8 n a^{2}\left(1+k \rho\left(x_{2}\right)\right)\right) \\
& \leq \frac{a^{2}}{c}(24+8 n(1+k a)) \\
& \leq \frac{a^{2}}{c}(24(1+k a)+8 n(1+k a)) \\
& =\tilde{c} a^{2}(1+k a),
\end{aligned}
$$

onde $\tilde{c}$ é uma constante que depende apenas de $c$ e $n$, sendo dada por $\tilde{c}=\frac{24+8 n}{c}$.

Assuma agora que $x_{2}$ é um ponto de corte de $x_{0}$. Seja $\sigma$ um segmento com velocidade unitária de $x_{0}$ a $x_{2}$ e $\bar{x}_{0} \in \sigma$ tal que $d\left(x_{0}, \bar{x}_{0}\right)=\epsilon$, para $\epsilon>0$ suficientemente pequeno. Então a função $\bar{\rho}(x)=d\left(x, \bar{x}_{0}\right)$ é suave, numa vizinhança próxima a $x_{2}$ e a função

$$
G_{\epsilon}(x)=\left(a^{2}-(\bar{\rho}(x)+\epsilon)^{2}\right)^{2} g(x)
$$

é uma "função suporte"de $G$, ou seja, $G_{\epsilon}(x) \leq G(x)$ e $G_{\epsilon}\left(x_{2}\right)=G\left(x_{2}\right)$. Então $G_{\epsilon}$ atinge seu máximo em $x_{2}$. Fazendo um desenvolvimento similar ao anterior e tomando o limite para $\epsilon \rightarrow 0$, obtemos a mesma estimativa de (4.17). Como $x_{2}$ é o ponto de máximo de $G$ em $B_{a}\left(x_{0}\right)$ temos $G\left(x_{1}\right) \leq G\left(x_{2}\right)$, obtendo

$$
g\left(x_{1}\right) \leq \frac{\tilde{c} a^{2}(1+k a)}{\left(a^{2}-\rho^{2}\left(x_{1}\right)\right)^{2}} .
$$

Como $M^{n}$ é completa, fazendo $a \rightarrow \infty$ em (4.18), obtemos $g\left(x_{1}\right)=0$, o que é uma contradição, já que $0=g\left(x_{0}\right)<g\left(x_{1}\right)$, por hipótese.

Observação 4.6 Utilizando a mesma demonstração do Lema 4.5, com pequenas alterações, podemos estender o resultado para $\Delta g \geq c g^{\alpha}$, $\operatorname{com} \alpha>1$.

Agora estamos prontos para demonstrar o seguinte teorema.

Teorema 4.7 Sejam $M^{3}$ uma variedade Riemanniana tridimensional completa orientada $e$ $f: M^{3} \rightarrow \mathbb{R}^{4}$ uma imersão minima isométrica com curvatura de Gauss-Kronecker identicamente zero e com segunda forma fundamental nunca nula. Se a curvatura escalar é limitada 
inferiormente, então $f\left(M^{3}\right)$ pode ser escrito como um produto Euclideano $L^{2} \times \mathbb{R}$, onde $L^{2}$ é uma superfície mínima completa de $\mathbb{R}^{3}$ com curvatura Gaussiana limitada inferiormente.

Demonstração: Sem perda de generalidade, podemos assumir que $M^{3}$ é simplesmente conexa, após passar para o espaço de recobrimento universal. Já que $M^{3}$ é simplesmente conexa, o referencial ortonormal $\left\{e_{1}, e_{2}, e_{3}\right\}$ de direções principais fica globalmente definido. Desta forma, temos que $M^{3}$ possui três curvaturas principais distintas $\lambda>0>-\lambda$, e a curvatura de Ricci é limitada inferiormente. As funções $u$ e $v$ estão bem definidas em $M^{3}$ inteira. Além disso, afirmamos que $u \mathrm{e}$ $v$ são funções harmônicas. De fato, por (4.3),

$$
\begin{aligned}
\Delta v= & \sum_{i=1}^{3} v_{i ; i}=\sum_{i=1}^{3}\left(e_{i}\left(v_{i}\right)+\sum_{j=1}^{3} v_{j} \omega_{j i}\left(e_{i}\right)\right)=\sum_{i=1}^{3}\left(e_{i} e_{i}(v)+\sum_{j=1}^{3} e_{j}(v) \omega_{j i}\left(e_{i}\right)\right) \\
= & e_{1} e_{1}(v)+e_{1}(v) \omega_{11}\left(e_{1}\right)+e_{2}(v) \omega_{21}\left(e_{1}\right)+e_{3}(v) \omega_{31}\left(e_{1}\right) \\
& +e_{2} e_{2}(v)+e_{1}(v) \omega_{12}\left(e_{2}\right)+e_{2}(v) \omega_{22}\left(e_{2}\right)+e_{3}(v) \omega_{32}\left(e_{2}\right) \\
& +e_{3} e_{3}(v)+e_{1}(v) \omega_{13}\left(e_{3}\right)+e_{2}(v) \omega_{23}\left(e_{3}\right)+e_{3}(v) \omega_{33}\left(e_{3}\right) \\
= & e_{1} e_{1}(v)+e_{2} e_{2}(v)+e_{3} e_{3}(v)+\left(\omega_{12}\left(e_{2}\right)+\omega_{13}\left(e_{3}\right)\right) e_{1}(v) \\
& +\left(\omega_{21}\left(e_{1}\right)+\omega_{23}\left(e_{3}\right)\right) e_{2}(v)+\left(\omega_{31}\left(e_{1}\right)+\omega_{32}\left(e_{2}\right)\right) e_{3}(v) \\
= & e_{1} e_{1}(v)+e_{2} e_{2}(v)+e_{3} e_{3}(v)+\left(0-\frac{1}{2} e_{1}(\ln \lambda)\right) e_{1}(v) \\
& +(-v-v) e_{2}(v)+\left(-\frac{1}{2} e_{3}(\ln \lambda)+0\right) e_{3}(v) \\
= & e_{1} e_{1}(v)+e_{2} e_{2}(v)+e_{3} e_{3}(v)-\frac{1}{2} e_{1}(\ln \lambda) e_{1}(v)-2 v e_{2}(v)-\frac{1}{2} e_{3}(\ln \lambda) e_{3}(v) .
\end{aligned}
$$

Note que, por (4.5), valem

$$
\begin{aligned}
e_{1} e_{1}(v) & =-e_{1} e_{3}(u) \\
e_{2} e_{2}(v) & =e_{2}\left(v^{2}-u^{2}\right)=2 v e_{2}(v)-2 u e_{2}(u) \\
& =2 v\left(v^{2}-u^{2}\right)-2 u(2 u v)=2 v^{3}-6 v u^{2} \\
e_{3} e_{3}(v) & =e_{3} e_{1}(u)
\end{aligned}
$$

Logo, por (4.4) e (4.5), temos

$$
\begin{aligned}
\Delta v & =e_{1} e_{1}(v)+e_{2} e_{2}(v)+e_{3} e_{3}(v)-\frac{1}{2} e_{1}(\ln \lambda) e_{1}(v)-2 v e_{2}(v)-\frac{1}{2} e_{3}(\ln \lambda) e_{3}(v) \\
& =-e_{1} e_{3}(u)+2 v^{3}-6 v u^{2}+e_{3} e_{1}(u)-\frac{1}{2} e_{1}(\ln \lambda) e_{1}(v)-2 v\left(v^{2}-u^{2}\right)-\frac{1}{2} e_{3}(\ln \lambda) e_{3}(v) \\
& =-\left[e_{1}, e_{3}\right](u)+\frac{1}{2} e_{1}(\ln \lambda) e_{3}(u)-\frac{1}{2} e_{3}(\ln \lambda) e_{1}(u)-4 v u^{2} \\
& =-\left(-\frac{1}{2} e_{3}(\ln \lambda) e_{1}(u)-2 u e_{2}(u)+\frac{1}{2} e_{1}(\ln \lambda) e_{3}(u)\right)+\frac{1}{2} e_{1}(\ln \lambda) e_{3}(u)-\frac{1}{2} e_{3}(\ln \lambda) e_{1}(u)-4 v u^{2} \\
& =0
\end{aligned}
$$

mostrando que $v$ é uma função harmônica. Para $u$, temos

$$
\begin{aligned}
\Delta u= & \sum_{i=1}^{3} u_{i ; i}=\sum_{i=1}^{3}\left(e_{i}\left(u_{i}\right)+\sum_{j=1}^{3} u_{j} \omega_{j i}\left(e_{i}\right)\right)=\sum_{i=1}^{3}\left(e_{i} e_{i}(u)+\sum_{j=1}^{3} e_{j}(u) \omega_{j i}\left(e_{i}\right)\right) \\
= & e_{1} e_{1}(u)+e_{1}(u) \omega_{11}\left(e_{1}\right)+e_{2}(u) \omega_{21}\left(e_{1}\right)+e_{3}(u) \omega_{31}\left(e_{1}\right) \\
& +e_{2} e_{2}(u)+e_{1}(u) \omega_{12}\left(e_{2}\right)+e_{2}(u) \omega_{22}\left(e_{2}\right)+e_{3}(u) \omega_{32}\left(e_{2}\right) \\
& +e_{3} e_{3}(u)+e_{1}(u) \omega_{13}\left(e_{3}\right)+e_{2}(u) \omega_{23}\left(e_{3}\right)+e_{3}(u) \omega_{33}\left(e_{3}\right) \\
= & e_{1} e_{1}(u)+e_{2} e_{2}(u)+e_{3} e_{3}(u)+\left(\omega_{12}\left(e_{2}\right)+\omega_{13}\left(e_{3}\right)\right) e_{1}(u) \\
& +\left(\omega_{21}\left(e_{1}\right)+\omega_{23}\left(e_{3}\right)\right) e_{2}(u)+\left(\omega_{31}\left(e_{1}\right)+\omega_{32}\left(e_{2}\right)\right) e_{3}(u) \\
= & e_{1} e_{1}(u)+e_{2} e_{2}(u)+e_{3} e_{3}(u)+\left(0-\frac{1}{2} e_{1}(\ln \lambda)\right) e_{1}(u) \\
& +(-v-v) e_{2}(u)+\left(-\frac{1}{2} e_{3}(\ln \lambda)+0\right) e_{3}(u)
\end{aligned}
$$




$$
=e_{1} e_{1}(u)+e_{2} e_{2}(u)+e_{3} e_{3}(u)-\frac{1}{2} e_{1}(\ln \lambda) e_{1}(u)-2 v e_{2}(u)-\frac{1}{2} e_{3}(\ln \lambda) e_{3}(u) .
$$

Note que, por (4.5), valem

$$
\begin{aligned}
e_{1} e_{1}(u) & =e_{1} e_{3}(v), \\
e_{2} e_{2}(u) & =e_{2}(2 u v)=2 u e_{2}(v)+2 v e_{2}(u) \\
& =2 u\left(v^{2}-u^{2}\right)+2 v(2 u v)=6 u v^{2}-2 u^{3}, \\
e_{3} e_{3}(u) & =-e_{3} e_{1}(v) .
\end{aligned}
$$

Logo, por (4.4) e (4.5), temos

$$
\begin{aligned}
\Delta u & =e_{1} e_{1}(u)+e_{2} e_{2}(u)+e_{3} e_{3}(u)-\frac{1}{2} e_{1}(\ln \lambda) e_{1}(u)-2 v e_{2}(u)-\frac{1}{2} e_{3}(\ln \lambda) e_{3}(u) \\
& =e_{1} e_{3}(v)+6 u v^{2}-2 u^{3}-e_{3} e_{1}(v)-\frac{1}{2} e_{1}(\ln \lambda) e_{1}(u)-2 v(2 u v)-\frac{1}{2} e_{3}(\ln \lambda) e_{3}(u) \\
& =\left[e_{1}, e_{3}\right](v)+2 u v^{2}-2 u^{3}-\frac{1}{2} e_{1}(\ln \lambda) e_{1}(u)-\frac{1}{2} e_{3}(\ln \lambda) e_{3}(u) \\
& =-\frac{1}{2} e_{3}(\ln \lambda) e_{1}(v)-2 u e_{2}(v)+\frac{1}{2} e_{1}(\ln \lambda) e_{3}(v)+2 u v^{2}-2 u^{3}-\frac{1}{2} e_{1}(\ln \lambda) e_{3}(v)+\frac{1}{2} e_{3}(\ln \lambda) e_{1}(v) \\
& =0,
\end{aligned}
$$

mostrando que $u$ também é uma função harmônica. Desta forma, por (4.5), temos

$$
\begin{aligned}
\Delta\left(u^{2}+v^{2}\right) & =\Delta\left(u^{2}\right)+\Delta\left(v^{2}\right) \\
& =2 u \Delta u+2\|\operatorname{grad} u\|^{2}+2 v \Delta v+2\|\operatorname{grad} v\|^{2} \\
& =2\left(\|\operatorname{grad} u\|^{2}+\|\operatorname{grad} v\|^{2}\right) \\
& \geq 2\left(\left(e_{2}(u)\right)^{2}+\left(e_{2}(v)\right)^{2}\right) \\
& =2\left(4 u^{2} v^{2}+\left(v^{2}-u^{2}\right)^{2}\right)
\end{aligned}
$$

ou seja,

$$
\Delta\left(u^{2}+v^{2}\right) \geq 2\left(u^{2}+v^{2}\right)^{2} .
$$

Pelo Lema 4.5, temos que $u^{2}+v^{2}$ é identicamente zero. Portanto $u=v \equiv 0$ e $\lambda$ é constante ao longo de curvas integrais de $e_{2}$. Considere a distribuição bidimensional $V$ gerada por $e_{1}$ e $e_{3}$. Como $u \equiv 0$, por (4.4) temos que $V$ é involutiva. Seja $L_{x}^{2}$ uma subvariedade integral maximal de $V$ passando pelo ponto $x \in M^{3}$, e denote por $i: L_{x}^{2} \rightarrow M^{3}$ sua aplicação inclusão. Então $\tilde{f}=f \circ i: L_{x}^{2} \rightarrow \mathbb{R}^{4}$ define uma imersão. Sejam $A_{1}$ e $A_{2}$ as segundas formas fundamentais de $\tilde{f}$ associados a $d f\left(e_{2}\right)$ e $\xi$, respectivamente. Uma conta direta mostra que $A_{1}=0 \mathrm{e}$

$$
A_{2} \sim\left(\begin{array}{cc}
\lambda & 0 \\
0 & -\lambda
\end{array}\right)
$$

com respeito à base $\left\{e_{1}, e_{3}\right\}$. Assim, $\tilde{f}: L_{x}^{2} \rightarrow \mathbb{R}^{4}$ é uma superfície mínima com curvatura Gaussiana limitada que se encontra em $\mathbb{R}^{3}$ e $d f\left(e_{2}\right)$ é constante ao longo de $\tilde{f}$. Portanto, $f\left(M^{3}\right)$ se quebra na forma desejada, e isso completa a demonstração do teorema.

Precisaremos do seguinte resultado para demonstrar o segundo teorema presente em Hasanis et al. [17].

Teorema 4.8 (Teorema da Curvatura Principal de Smyth e Xavier [27]) Seja $M^{n}$ um hipersuperfície orientável e completa imersa em $\mathbb{R}^{n+1}$, que não é um hiperplano. Seja $\Lambda \subset \mathbb{R}$ o conjunto dos valores não nulos assumidos pelos autovalores de $h$ e seja $\Lambda^{ \pm}=\Lambda \cap \mathbb{R}^{ \pm}$.

(i) Se nem $\Lambda^{+}$nem $\Lambda^{-}$são vazios, então $\inf \Lambda^{+}=\sup \Lambda^{-}=0$.

(ii) $S e \Lambda^{+}$ou $\Lambda^{-}$são vazios, então o fecho $\bar{\Lambda}$ de $\Lambda$ é conexo. 
Agora estamos prontos para demonstrar o último resultado.

Teorema 4.9 Sejam $M^{3}$ uma variedade Riemanniana tridimensional completa orientada $e$ $f: M^{3} \rightarrow \mathbb{R}^{4}$ uma imersão mínima isométrica com curvatura de Gauss-Kronecker constante. Então a curvatura de Gauss-Kronecker é identicamente zero.

Demonstração: Podemos escolher uma orientação tal $\lambda_{1}>0>\lambda_{2} \geq \lambda_{3}$. Pelo Teorema 4.8, temos que $\inf \left(\Lambda \cap \mathbb{R}^{+}\right)=0$, onde $\Lambda$ é o conjunto de valores assumidos pelas curvaturas principais diferentes de zero. Portanto existe uma sequência de pontos $\left\{x_{n}\right\}$ tal que $\lambda_{1}\left(x_{n}\right) \rightarrow 0$. Pela minimalidade obtemos $\lambda_{1} \geq\left|\lambda_{i}\right|, i=2,3$, e consequentemente a curvatura de Gauss-Kronecker satisfaz $K\left(x_{n}\right) \rightarrow 0$. Logo $K$ é zero, e concluímos a demonstração. 


\section{Referências Bibliográficas}

[1] Asperti, A. C., Chaves, R. M. B. e Sousa Jr., L. A. M. The Gauss-Kronecker curvature of minimal hypersurfaces in four-dimensional space forms. Mathematische Zeitschrift, 267:523533, 2011.

[2] Boothby, W. M. An introduction to differentiable manifolds and Riemannian geometry. Academic press, 1986.

[3] Cheeger, J. e Gromoll, D. The splitting theorem for manifolds of nonnegative Ricci curvature. Journal of Differential Geometry, 6:119-128, 1971.

[4] Cheng, Q.-M. Curvatures of complete hypersurfaces in space forms. Proceedings of the Royal Society of Edinburgh, 134:55-68, 2004.

[5] Cheng, S.-Y. e Yau, S.-T. Maximal space-like hypersurfaces in the Lorentz-Minkowski spaces. Annals of Mathematics, 104:407-419, 1976.

[6] Chern, S.-S., do Carmo, M. e Kobayashi, S. Minimal submanifolds of a sphere with second fundamental form of constant length. Em Functional analysis and related fields, páginas 59-75. Springer, 1970.

[7] Chodosh, O., Eichmair, M. e Moraru, V. A splitting theorem for scalar curvature. Communications on Pure and Applied Mathematics, 72:1231-1242, 2019.

[8] Dajczer, M. e Gromoll, D. Gauss parametrizations and rigidity aspects of submanifolds. Journal of Differential Geometry, 22:1-12, 1985.

[9] Dajczer, M., Kasioumis, T., Savas-Halilaj, A. e Vlachos, T. Complete minimal submanifolds with nullity in Euclidean space. Mathematische Zeitschrift, 287:481-491, 2017.

[10] Dajczer, M., Kasioumis, T., Savas-Halilaj, A. e Vlachos, T. Complete minimal submanifolds with nullity in Euclidean spheres. Commentarii Mathematici Helvetici, 93:645-660, 2018.

[11] Dajczer, M., Kasioumis, T., Savas-Halilaj, A. e Vlachos, T. Complete minimal submanifolds with nullity in the hyperbolic space. The Journal of Geometric Analysis, 29:413-427, 2019.

[12] de Almeida, S. C. e Brito, F. G. B. Minimal hypersurfaces of $\mathbb{S}^{4}$ with constant Gauss-Kronecker curvature. Mathematische Zeitschrift, 195:99-107, 1987.

[13] do Carmo, M. P. Geometria Riemanniana. Projeto Euclides. Instituto Nacional de Matemática Pura e Aplicada, $5^{\mathrm{a}}$ edição, 2015.

[14] do Carmo, M. P. O Método do Referencial Móvel. Publicações Matemáticas. Instituto Nacional de Matemática Pura e Aplicada, $2^{\mathrm{a}}$ edição, 2012.

[15] do Carmo, M. e Warner, F. Rigidity and convexity of hypersurfaces in spheres. Journal of Differential Geometry, 4:133-144, 1970.

[16] Hadamard, J. Les surfaces à courbures opposées et leurs lignes géodésique. Journal de Mathématiques Pures et Appliquées, 4:27-73, 1898. 
[17] Hasanis, T., Savas-Halilaj, A. e Vlachos, T. Minimal hypersurfaces with zero Gauss-Kronecker curvature. Illinois Journal of Mathematics, 49:523-529, 2005.

[18] Hasanis, T., Savas-Halilaj, A. e Vlachos, T. Complete minimal hypersurfaces in the hyperbolic space $\mathbb{H}^{4}$ with vanishing Gauss-Kronecker curvature. Transactions of the American Mathematical Society, 359:2799-2818, 2007.

[19] Hasanis, T., Savas-Halilaj, A. e Vlachos, T. Complete minimal hypersurfaces in $\mathbb{S}^{4}$ with zero Gauss-Kronecker curvature. Mathematical Proceedings of the Cambridge Philosophical Society, 142:125-132, 2007.

[20] Innami, N. Splitting theorems of Riemannian manifolds. Compositio Mathematica, 47:237-247, 1982.

[21] Nadirashvili, N. Hadamard's and Calabi-Yau's conjectures on negatively curved and minimal surfaces. Inventiones Mathematicae, 126:457-465, 1996.

[22] Nishikawa, S. On maximal spacelike hypersurfaces in a Lorentzian manifold. Nagoya Mathematical Journal, 95:117-124, 1984.

[23] Omori, H. Isometric immersions of Riemannian manifolds. Journal of the Mathematical Society of Japan, 19:205-214, 1967.

[24] Peng, C.-K. e Terng, C.-L. Minimal hypersurfaces of spheres with constant scalar curvature. Annals of Mathematics Studies, páginas 177-198, 1983.

[25] Ramanathan, J. Minimal hypersurfaces in $\mathbb{S}^{4}$ with vanishing Gauss-Kronecker curvature. Mathematische Zeitschrift, 205:645-658, 1990.

[26] Simons, J. Minimal varieties in Riemannian manifolds. Annals of Mathematics, 88:62-105, 1968.

[27] Smyth, B. e Xavier, F. Efimov's theorem in dimension greater than two. Inventiones Mathematicae, 90:443-450, 1987.

[28] Suzuki, C. d. A. Hipersuperficies de rotação em espaços de curvatura constante. Dissertação de Mestrado, Universidade de São Paulo, 1989.

[29] Takegoshi, K. Strongly $p$-subharmonic functions and volume growth property of complete Riemannian manifolds. Osaka Journal of Mathematics, 38:839-850, 2001.

[30] Toponogov, V. A. Riemannian spaces which contain straight lines. American Mathematical Society Translations: Series 2, 37:287-290, 1964.

[31] Van der Waerden, B. L. Algebra, volume 1. Springer-Verlag New York, 1991.

[32] Yau, S.-T. Harmonic functions on complete riemannian manifolds. Communications on Pure and Applied Mathematics, 28:201-228, 1975. 


\section{Índice Remissivo}

Aplicação

Diferenciável, 3

Normal, 37

\section{Campo de Vetores}

Normal, 25

Tangente, 4

Colchete de Lie, 9

Correferencial Associado, 4

Curvatura

de Gauss-Kronecker, 28

de Ricci, 22, 29

Escalar, 22, 29

Média, 27

Principal, 27

Seccional, 19

Delta de Kronecker, 4

Diferencial Covariante, 15

Distribuição, 14

Completamente Integrável, 14

Involutiva, 14

Divergência, 23

Equação

de Codazzi, 29

de Gauss, 28

de Ricci, 31

Espaço

de De Sitter, 36-37

Forma, 35

Hiperbólico, 36

Lorentziano, 36

Normal, 25

Tangente, 3

Fibrado

Normal, 36

Fibrado Tangente, 4

Formas

de Conexão, 6

de Curvatura, 7
Hessiano, 23

Hipersuperfície, 25

Imersão, 4

Estacionária, 37

Geodésica, 26

Isométrica, 24

Mínima, 28

Riemanniana, 24

Tipo-Espaço, 36

Laplaciano, 23

Lema

de Cartan, 5

de Levi-Civitta, 6

Operador de Curvatura, 16

Operadores Diferenciais, 22

Referencial Ortonormal

Adaptado, 24

Móvel, 4

Segunda Forma Fundamental, 26

Super-harmônica, 39

Tensor, 14

Curvatura, 18

de Ricci, 22

Teorema de Frobenius, 14

Variedade

Diferenciável, 3

Integral, 14

Riemanniana, 4

Completa, 4

Gradiente, 22 